Supplement of Clim. Past, 10, 2237-2252, 2014

http://www.clim-past.net/10/2237/2014/

doi:10.5194/cp-10-2237-2014-supplement

(C) Author(s) 2014. CC Attribution 3.0 License.

(c) (i)

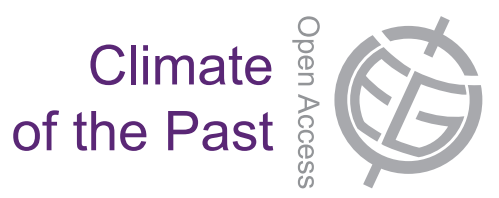

Supplement of

\title{
Implication of methodological uncertainties for mid-Holocene sea surface temperature reconstructions
}

I. Hessler et al.

Correspondence to: I. Hessler (ines.hessler@mq.edu.au) 


\section{Supplementary Material}

Implication of sensor and analytical uncertainties for mid-Holocene sea surface temperature reconstructions

Ines Hessler1,2, *, Sandy P. Harrison1,3, Michal Kuchera2, Claire Waelbroeck4, Min-Te

Chen $^{5}$, Carin Anderson 6 , Anne de Vernal7 , Bianca Fréchette ${ }^{7}$, Angela Cloke-Hayes ${ }^{8}$,

Guillaume Leduc ${ }^{9,10}$, and Laurent Londeix ${ }^{11}$

The SI contains (a) details of the sites used in these analyses (SI Table 1) and references to the primary data, (b) details of the reconstructions at individual sites (SI Table 2, 3, and 4), and (c) grid cell reconstruction (SI Table 5). It also contains seasonal plots showing the impact of using different baseline climates on seasonal sea-surface temperature (SST) reconstructions (SI Figure 1 and 2) and the impact of using different time windows (SI Figure 3 and 4). We also include plots showing the reconstructions obtained from different sensor along latitudinal gradients within individual ocean basins (SI Figure 5).

All data including additional information on the age models of previously unpublished data sets are available on www.pangaea.de. The complete data set can be accessed via doi:10.1594/PANGAEA.830811, while the age models for previously unpublished records are available via doi.pangaea.de/10.1594/PANGAEA.830814. 
Table 1: MH sea surface temperature records used in this study

\begin{tabular}{|c|c|c|c|c|c|c|c|}
\hline Core ID & Lat. $\left[^{\circ}\right]-\mathrm{S},+\mathrm{N}$ & Long. $\left[^{\circ}\right]-W,+E$ & Ocean & $\begin{array}{l}\text { water depth } \\
\text { [mbsf] }\end{array}$ & Proxy & Reference & Study \\
\hline MD85674 & 3.18 & 50.43 & Indian Ocean & 4875 & Alkenone & Bard et al., 1997 & GHOST \\
\hline MD77194 & 10.47 & 75.23 & Indian Ocean & 1222 & Alkenone & $\begin{array}{l}\text { Sonzogni et al., } \\
1998\end{array}$ & GHOST \\
\hline TY93-905 & 11.07 & 51.95 & Indian Ocean & 1567 & Alkenone & Kim et al., 2004 & GHOST \\
\hline SO93-126KL & 19.97 & 90.03 & Indian Ocean & 1253 & Alkenone & $\begin{array}{l}\text { Kudrass et al., } \\
2001\end{array}$ & GHOST \\
\hline SO90-93KL & 23.58 & 64.22 & Indian Ocean & 1802 & Alkenone & $\begin{array}{l}\text { Schulz et al., } \\
\text { 2002; Emeis, } \\
\text { unpublished data }\end{array}$ & GHOST \\
\hline MD05-2928 & -11.29 & 148.86 & Pacific & 2250 & Alkenone & Shiau et al., 2011 & this study \\
\hline SO139-74KL & -6.54 & 103.83 & Indian Ocean & 1690 & Alkenone & $\begin{array}{l}\text { Lückge et al., } \\
2009\end{array}$ & GHOST \\
\hline GeoB10038-4 & -5.94 & 103.25 & Indian Ocean & 1819 & Alkenone & $\begin{array}{l}\text { Mohtadi et al., } \\
2010\end{array}$ & this study \\
\hline $18287-3$ & 5.65 & 110.65 & Indian Ocean & 598 & Alkenone & $\begin{array}{l}\text { Kienast et al., } \\
2001\end{array}$ & GHOST \\
\hline GIK18287-3 & 5.98 & 110.65 & Indian Ocean & 598 & Alkenone & $\begin{array}{l}\text { Steinke et al., } \\
2001\end{array}$ & this study \\
\hline 17964 & 6.16 & 112.21 & Indian Ocean & 1556 & Alkenone & $\begin{array}{l}\text { Pelejero et al., } \\
\text { 1999a,b; Wang et } \\
\text { al., } 1999\end{array}$ & GHOST \\
\hline MD97-2151 & 8.73 & 109.87 & Indian Ocean & 1598 & Alkenone & Zhao et al., 2006 & GHOST \\
\hline $18252-3$ & 9.23 & 109.38 & Indian Ocean & 1273 & Alkenone & $\begin{array}{l}\text { Kienast et al., } \\
2001\end{array}$ & GHOST \\
\hline MD01-2392 & 9.85 & 110.21 & Indian Ocean & 1966 & Alkenone & Xie et al., 2007 & this study \\
\hline
\end{tabular}




\begin{tabular}{|c|c|c|c|c|c|c|c|}
\hline ODP977 & 36.03 & -1.96 & $\begin{array}{c}\text { Mediterranean } \\
\text { Sea }\end{array}$ & 1984 & Alkenone & $\begin{array}{l}\text { Martrat et al., } \\
2004\end{array}$ & GHOST \\
\hline MD95-2043 & 36.14 & -2.62 & $\begin{array}{c}\text { Mediterranean } \\
\text { Sea }\end{array}$ & 1841 & Alkenone & Cacho et al., 1999 & GHOST \\
\hline RL11 & 36.75 & 17.72 & $\begin{array}{c}\text { Mediterranean } \\
\text { Sea }\end{array}$ & 3376 & Alkenone & Emeis et al., 2000 & GHOST \\
\hline $\begin{array}{l}\text { M40-4- } \\
\text { SL78/78MUC8 }\end{array}$ & 37.04 & 13.19 & $\begin{array}{l}\text { Mediterranean } \\
\text { Sea }\end{array}$ & 470 & Alkenone & $\begin{array}{l}\text { Emeis and } \\
\text { Dawson, 2003b; } \\
\text { Emeis and Schulz, } \\
\text { unpublished data }\end{array}$ & GHOST \\
\hline BS79-33 & 38.26 & 14.03 & $\begin{array}{l}\text { Mediterranean } \\
\text { Sea }\end{array}$ & 1282 & Alkenone & Cacho et al., 2001 & GHOST \\
\hline BS79-38 & 38.41 & 13.58 & $\begin{array}{c}\text { Mediterranean } \\
\text { Sea }\end{array}$ & 1489 & Alkenone & Cacho et al., 2002 & GHOST \\
\hline M44-KL71 & 40.84 & 27.76 & $\begin{array}{l}\text { Mediterranean } \\
\text { Sea }\end{array}$ & 566 & Alkenone & $\begin{array}{l}\text { Sperling et al., } \\
2003\end{array}$ & GHOST \\
\hline AD91-17 & 40.87 & 18.64 & $\begin{array}{l}\text { Mediterranean } \\
\text { Sea }\end{array}$ & 844 & Alkenone & $\begin{array}{l}\text { Giunta et al., } \\
2001\end{array}$ & GHOST \\
\hline GeoB4905-4 & 2.50 & 9.39 & North Atlantic & 1328 & Alkenone & $\begin{array}{l}\text { Weldeab et al., } \\
2007\end{array}$ & GHOST \\
\hline ODP1002C & 10.71 & -65.17 & North Atlantic & 893 & Alkenone & $\begin{array}{l}\text { Herbert and } \\
\text { Schuffert, 2000; } \\
\text { Peterson et al., } \\
2000\end{array}$ & GHOST \\
\hline M35003-4 & 12.08 & -61.25 & North Atlantic & 1299 & Alkenone & $\begin{array}{l}\text { Rühlemann et al., } \\
1999\end{array}$ & GHOST \\
\hline $74 \mathrm{KL}$ & 14.32 & 57.35 & North Atlantic & 3212 & Alkenone & Kim et al., 2004 & GHOST \\
\hline BOFS 31K & 19.00 & -20.17 & North Atlantic & 3300 & Alkenone & $\begin{array}{l}\text { Zhao et al., 1995; } \\
\text { Chapmann et al., } \\
1996\end{array}$ & GHOST \\
\hline GeoB7926-2 & 20.22 & -18.45 & North Atlantic & 2500 & Alkenone & $\begin{array}{l}\text { Romero et al., } \\
2008\end{array}$ & GHOST \\
\hline ODP658C & 20.75 & -18.58 & North Atlantic & 2263 & Alkenone & Zhao et al., 1995, & GHOST \\
\hline
\end{tabular}




\begin{tabular}{|l} 
GeoB5546-2 \\
GeoB5844-2 \\
GeoB6007 \\
ODP969E \\
ODP967D \\
M39-008 \\
GeoB5901-2 \\
CH07-98-GGC19 \\
SU81-18 \\
MD95-2042 \\
MD01-2443 \\
D13882 \\
OCE326-GGC26 \\
OCE326-GGC30 \\
IOW225517 \\
IOW225514 \\
MD95-2015 \\
MD95-2011 \\
JR51-GC35 \\
M23258-2
\end{tabular}

deMenocal et al.,

2000

Freudenthal,

unpublished

Alkenone

Alkenone

Arz et al., 2003

GHOST

Alkenone

Emeis et al.,

2003c

Alkenone

Emeis et al., 2000

GHOST

Alkenone

Alkenone

Kim et al., 2004

Sachs et al., 2007

Alkenone

Alkenone

Bard et al., 2000

Pailler and Bard,

2002

Alkenone

Alkenone

Martrat et al.,

2007

North Atlantic $\quad 3975$

North Atlantic

2009

Alkenone

Alkenone

North Atlantic

Alkenone

North Atlantic

Alkenone

North Atlantic

Alkenone

North Atlantic

Alkenone

North Atlantic

Alkenone

Sachs et al., 2007

Sachs et al., 2007

Emeis et al.,

2003a

Emeis et al.,

2003a

2630
1048

Marchal et al.,

2002

Calvo et al., 2002

Bendle and

Rosell-Mélé, 2007

GHOST

GHOST

GHOST

GHOST

GHOST

GHOST

GHOST

GHOST

GHOST

GHOST

GHOST

GHOST

GHOST

GHOST

GHOST

$\begin{array}{ll}\text { North Atlantic } & 1768\end{array}$

Alkenone

Marchal et al.

GHOST

GHOST 


\begin{tabular}{|c|c|c|}
\hline MD97-2120 & -45.53 & 174.93 \\
\hline S0136-GC11 & -43.44 & 167.85 \\
\hline ODP1233 & -41.01 & -74.45 \\
\hline GeoB3313-1 & -41.00 & -74.45 \\
\hline MD97-2121 & -40.38 & 177.99 \\
\hline GIK17748-2 & -32.75 & -72.03 \\
\hline GeoB7139-2 & -30.20 & -71.98 \\
\hline V19-30 & -3.38 & -83.52 \\
\hline V19-28 & -2.51 & -84.65 \\
\hline V21-30 & -1.22 & -89.68 \\
\hline V19-27 & -0.47 & -82.67 \\
\hline ME0005A-24JC & 1.50 & -86.49 \\
\hline KNR176-JPC32 & 4.85 & -77.96 \\
\hline MD02-2529 & 8.21 & -84.12 \\
\hline SCS90-36 & 18.00 & 111.49 \\
\hline 17940-2 & 20.12 & 117.38 \\
\hline
\end{tabular}

\begin{tabular}{|c|c|c|c|}
\hline & & & $\begin{array}{l}\text { 2002; Sarnthein } \\
\text { et al., } 2003\end{array}$ \\
\hline Pacific & 1210 & Alkenone & $\begin{array}{l}\text { Pahnke and } \\
\text { Sachs, } 2006\end{array}$ \\
\hline Pacific & 1556 & Alkenone & $\begin{array}{l}\text { Barrows et al., } \\
2008\end{array}$ \\
\hline Pacific & 838 & Alkenone & $\begin{array}{l}\text { Lamy et al., 2004; } \\
\text { Kaiser et al., } \\
\text { 2005, Lamy et al., } \\
2007\end{array}$ \\
\hline Pacific & 852 & Alkenone & Lamy et al., 2002 \\
\hline Pacific & 3014 & Alkenone & $\begin{array}{l}\text { Pahnke and } \\
\text { Sachs, } 2006\end{array}$ \\
\hline Pacific & 2545 & Alkenone & Kim et al., 2002b \\
\hline Pacific & 3270 & Alkenone & Kaiser et al., 2008 \\
\hline Pacific & 3091 & Alkenone & $\begin{array}{l}\text { Koutavas and } \\
\text { Sachs, } 2008\end{array}$ \\
\hline Pacific & 2720 & Alkenone & $\begin{array}{l}\text { Koutavas and } \\
\text { Sachs, } 2008\end{array}$ \\
\hline Pacific & 617 & Alkenone & $\begin{array}{l}\text { Koutavas and } \\
\text { Sachs, } 2008\end{array}$ \\
\hline Pacific & 1373 & Alkenone & $\begin{array}{l}\text { Koutavas and } \\
\text { Sachs, } 2008\end{array}$ \\
\hline Pacific & 2941 & Alkenone & $\begin{array}{l}\text { Kienast et al., } \\
2006\end{array}$ \\
\hline Pacific & 2200 & Alkenone & $\begin{array}{l}\text { Pahnke et al., } \\
2007\end{array}$ \\
\hline Pacific & 1619 & Alkenone & Leduc et al., 2007 \\
\hline Pacific & 2050 & Alkenone & Huang et al., 1997 \\
\hline Pacific & 1727 & Alkenone & $\begin{array}{l}\text { Pelejero et al., } \\
\text { 1999a; Wang et } \\
\text { al., } 1999\end{array}$ \\
\hline
\end{tabular}

GHOST

GHOST

GHOST

GHOST

GHOST

GHOST

GHOST

GHOST

GHOST

GHOST

GHOST

GHOST

GHOST

GHOST

GHOST

GHOST 


\begin{tabular}{|c|c|c|c|c|c|c|c|}
\hline PC17 & 21.36 & -158.19 & Pacific & 503 & Alkenone & Lee et al., 2001 & GHOST \\
\hline LAPAZ21P & 22.99 & -109.47 & Pacific & 624 & Alkenone & $\begin{array}{l}\text { Herbert et al., } \\
2001\end{array}$ & GHOST \\
\hline ST.19 & 31.10 & 138.67 & Pacific & 3336 & Alkenone & $\begin{array}{l}\text { Sawada and } \\
\text { Handa, } 1998\end{array}$ & GHOST \\
\hline ODP1012B & 32.28 & -118.38 & Pacific & 1772 & Alkenone & $\begin{array}{l}\text { Herbert et al., } \\
2001\end{array}$ & GHOST \\
\hline ST.14 & 32.67 & 138.46 & Pacific & 3252 & Alkenone & $\begin{array}{l}\text { Sawada and } \\
\text { Handa, } 1998\end{array}$ & GHOST \\
\hline ODP1017E & 34.54 & -121.11 & Pacific & 955 & Alkenone & $\begin{array}{l}\text { Kennett et al., } \\
\text { 2000; Ostertag- } \\
\text { Henning and Stax, } \\
2000\end{array}$ & GHOST \\
\hline SSDP-102 & 34.95 & 128.88 & Pacific & 40 & Alkenone & Kim et al., 2004 & GHOST \\
\hline ODP1019C & 41.68 & -124.93 & Pacific & 980 & Alkenone & $\begin{array}{l}\text { Barron et al., } \\
2003\end{array}$ & GHOST \\
\hline W8709A-8TC & 42.54 & -127.68 & Pacific & 3111 & Alkenone & Prahl et al., 1995 & this study \\
\hline MD01-2412 & 44.53 & 145.04 & Pacific & 1225 & Alkenone & $\begin{array}{l}\text { Harada et al., } \\
2006\end{array}$ & GHOST \\
\hline MR00-K03-PC01 & 46.19 & 152.32 & Pacific & 2793 & Alkenone & $\begin{array}{l}\text { Harada et al., } \\
2004\end{array}$ & this study \\
\hline GGC15 & 48.17 & 151.34 & Pacific & 1980 & Alkenone & $\begin{array}{l}\text { Keigwin et al., } \\
\text { 1998; Ternois et } \\
\text { al., } 2000\end{array}$ & GHOST \\
\hline JT96-0909pc & 48.91 & -126.89 & Pacific & 920 & Alkenone & $\begin{array}{l}\text { Kienast and } \\
\text { McKay, } 2001\end{array}$ & GHOST \\
\hline PC4 & 49.49 & 146.13 & Pacific & 664 & Alkenone & Seki et al., 2004 & GHOST \\
\hline PC2 & 50.40 & 148.32 & Pacific & 1258 & Alkenone & Seki et al., 2004 & GHOST \\
\hline TN057-21PC & -41.13 & 8.80 & South Atlantic & 4981 & Alkenone & $\begin{array}{l}\text { Pahnke and } \\
\text { Sachs, 2006; } \\
\text { Sachs and } \\
\text { Anderson, } 2003\end{array}$ & GHOST \\
\hline
\end{tabular}




\begin{tabular}{|c|c|c|c|c|c|c|c|}
\hline GeoB1710-3 & -23.43 & 11.69 & South Atlantic & 1045 & Alkenone & Kirst et al., 1999 & this study \\
\hline GeoB1711-4 & -23.31 & 12.37 & South Atlantic & 1967 & Alkenone & Kirst et al., 1999 & this study \\
\hline GeoB1712-4 & -23.26 & 12.81 & South Atlantic & 998 & Alkenone & Kirst et al., 1999 & this study \\
\hline MD79257 & -20.40 & 36.33 & South Atlantic & 1262 & Alkenone & $\begin{array}{l}\text { Duplessy et al., } \\
\text { 1991; Bard et al., } \\
\text { 1997; Sonzogni et } \\
\text { al., } 1998\end{array}$ & GHOST \\
\hline GeoB1023-5 & -17.16 & 11.01 & South Atlantic & 1978 & Alkenone & Kim et al., 2002a & GHOST \\
\hline ODP1078C & -11.92 & 13.40 & South Atlantic & 426 & Alkenone & Kim et al., 2003 & GHOST \\
\hline GeoB6518-1 & -5.59 & 11.22 & South Atlantic & 962 & Alkenone & $\begin{array}{l}\text { Schefuss et al., } \\
2005\end{array}$ & GHOST \\
\hline GeoB 3129-1 & -4.61 & -36.64 & South Atlantic & 830 & Alkenone & $\begin{array}{l}\text { Kim, unpublished } \\
\text { data }\end{array}$ & GHOST \\
\hline GeoB3910-2 & -4.02 & -36.35 & South Atlantic & 2362 & Alkenone & $\begin{array}{l}\text { Jaeschke et al., } \\
2007\end{array}$ & GHOST \\
\hline MD94-103 & -45.58 & 86.53 & Southern Ocean & 3560 & Alkenone & Sicre et al., 2005 & GHOST \\
\hline MD03-2611 & -36.73 & 136.55 & Southern Ocean & 2420 & Alkenone & Calvo et al., 2007 & GHOST \\
\hline PL-96-112 & 71.27 & 42.61 & Arctic Sea & 286 & Dinocysts & $\begin{array}{l}\text { Voronina et } \\
\text { al.,2001; de } \\
\text { Vernal et al., } \\
\text { 2013a }\end{array}$ & this study \\
\hline HLY0501-05 & 72.69 & -157.52 & Arctic Sea & 415 & Dinocysts & $\begin{array}{l}\text { McKay et al., } \\
\text { 2008; de Vernal } \\
\text { et al., 2013a }\end{array}$ & this study \\
\hline HU-91-039-008 & 77.27 & -74.32 & Arctic Sea & 663 & Dinocysts & $\begin{array}{l}\text { Levac et al., 2001; } \\
\text { de Vernal and } \\
\text { Hillaire-Marcel, } \\
\text { 2006; de Vernal } \\
\text { et al., 2013a }\end{array}$ & this study \\
\hline MD95-2043 & 36.14 & -2.62 & $\begin{array}{l}\text { Mediterranean } \\
\text { Sea }\end{array}$ & 1841 & Dinocysts & $\begin{array}{l}\text { Ruis-Zargouni, } \\
2010\end{array}$ & this study \\
\hline ODP Leg 161 Site & 36.21 & -4.31 & Mediterranean & 1108 & Dinocysts & Combourieu- & this study \\
\hline
\end{tabular}




$\begin{aligned} & \text { M76 Hole C } \\ & \text { MD99-2346 } \\ & \text { MD01-2430 } \\ & \text { HU-90-031-044 } \\ & \text { MD-95-2033 } \\ & \text { HU-2003-033-011 } \\ & \text { MD-99-2220 } \\ & \text { HU-90-031-019 } \\ & \text { HU-91-045-094 }\end{aligned} \mid$

Sea Mediterranea

Sea

36.95

11.67

40.80

44.49

27.73

$-55.19$

44.66

$-55.62$

48.60

49.29

50.20

$-45.69$

North Atlantic

diterranea$$
\text { Sea }
$$

Mediterranean

$$
\text { Sea }
$$

North Atlantic

North Atlantic

North Atlantic

North Atlantic

North Atlantic
Dinocysts

Nebout et al.,

1999; 2002

Dinocysts Londeix et al.,

Dinocysts

Dinocysts

Dinocysts

Solignac et al.,

Dinocysts
Ruis-Zargouni,

2010; Ruis-

Zargouni et al.,

2010

Ruis-Zargouni,

2010; Ruis-

Zargouni et al.,

2010

2009

de Vernal and

Hillaire-Marcel,

2006

de Vernal and

Hillaire-Marcel,

2006

2013a

de Vernal et al.

2013a

de Simard and de

Vernal, 1998; de

Vernal et al.,

2013a

2004; de Vernal

and Hillaire-

Marcel, 2006; de

Vernal et al.,

2013a

this study

this study

this study

this study

this study

this study

this study

this study

this study

de Vernal and 


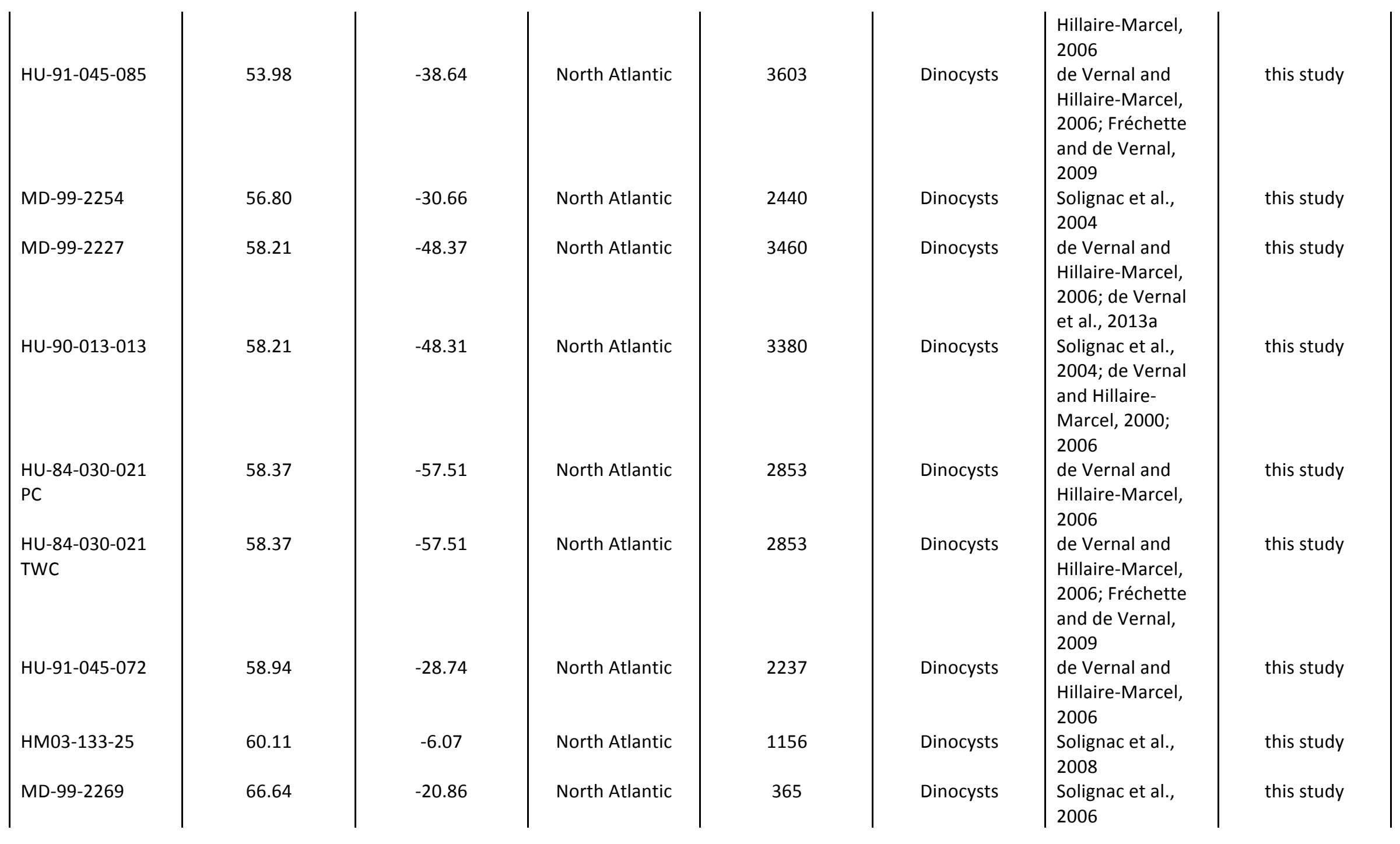




\begin{tabular}{|c|c|c|c|c|c|c|c|}
\hline GIK23323 & 67.77 & 5.92 & North Atlantic & 1286 & Dinocysts & $\begin{array}{l}\text { de Vernal et al., } \\
2013 a\end{array}$ & this study \\
\hline JM-96-1207 & 68.10 & -29.35 & North Atlantic & 404 & Dinocysts & $\begin{array}{l}\text { Solignac et al., } \\
2006\end{array}$ & this study \\
\hline 2005-804-006 & 68.99 & -106.57 & North Atlantic & 118 & Dinocysts & Ledu et al., 2010 & this study \\
\hline 2004-804-009 & 74.19 & -81.20 & North Atlantic & 781 & Dinocysts & Ledu et al., 2008 & this study \\
\hline 2005-804-004 & 74.27 & -91.09 & North Atlantic & 350 & Dinocysts & Ledu et al., 2010 & this study \\
\hline $\begin{array}{l}\text { GIK17748-2 } \\
\text { (SO80-4) }\end{array}$ & -32.75 & -72.03 & Pacific & 2545 & Forams & $\begin{array}{l}\text { Mohtadi and } \\
\text { Hebbeln, } 2004\end{array}$ & this study \\
\hline GeoB7139-2 & -30.20 & -71.98 & Pacific & 3269 & Forams & $\begin{array}{l}\text { Mohtadi and } \\
\text { Hebbeln, } 2004\end{array}$ & this study \\
\hline GeoB7112-5 & -24.03 & -70.82 & Pacific & 2507 & Forams & $\begin{array}{l}\text { Mohtadi et al., } \\
2004\end{array}$ & this study \\
\hline ODP 969 & 33.84 & 24.88 & Mediterranean & 2200 & Forams & $\begin{array}{l}\text { Hayes, } \\
\text { unpublished data }\end{array}$ & this study \\
\hline TTR17-MS419G & 35.45 & -4.62 & Mediterranean & 410 & Forams & $\begin{array}{l}\text { Margreth et al., } \\
2011\end{array}$ & this study \\
\hline KS310 & 35.55 & -1.57 & Mediterranean & 1900 & Forams & $\begin{array}{l}\text { Rohling et al., } \\
1995\end{array}$ & this study \\
\hline MD81-LC21 & 35.66 & 26.58 & Mediterranean & 1522 & Forams & $\begin{array}{l}\text { De Rijk et al., } \\
\text { 1999; Hayes et } \\
\text { al., } 1999\end{array}$ & this study \\
\hline ODP 973 & 35.78 & 18.95 & Mediterranean & 3695 & Forams & $\begin{array}{l}\text { Hayes, } \\
\text { unpublished data }\end{array}$ & this study \\
\hline MD 95-2043 & 36.14 & -2.62 & Mediterranean & 1841 & Forams & Cacho et al., 1999 & this study \\
\hline ODP 977 & 36.19 & -1.57 & Mediterranean & 1984 & Forams & $\begin{array}{l}\text { Pérez-Folgado et } \\
\text { al., 2003; Pérez- } \\
\text { Folgado et al., } \\
2004\end{array}$ & this study \\
\hline ODP 964 & 36.26 & 17.74 & Mediterranean & 3657 & Forams & $\begin{array}{l}\text { Hayes, } \\
\text { unpublished data }\end{array}$ & this study \\
\hline MD04-2797CQ & 36.95 & 11.67 & Mediterranean & 771 & Forams & $\begin{array}{l}\text { Rouis, Zargouni et } \\
\text { al., } 2010\end{array}$ & this study \\
\hline
\end{tabular}




\begin{tabular}{|c|c|c|c|c|c|c|c|}
\hline ODP 975 & 38.89 & 4.50 & Mediterranean & 2426 & Forams & $\begin{array}{l}\text { Hayes, } \\
\text { unpublished data }\end{array}$ & this study \\
\hline ODP 974 & 40.35 & 12.13 & Mediterranean & 3493 & Forams & $\begin{array}{l}\text { Hayes, } \\
\text { unpublished data }\end{array}$ & this study \\
\hline M40/4 $80 \mathrm{SL}$ & 40.95 & 11.00 & Mediterranean & 1881 & Forams & $\begin{array}{l}\text { Hayes, } \\
\text { unpublished data }\end{array}$ & this study \\
\hline IN68-9 & 41.78 & 17.90 & Mediterranean & 1234 & Forams & $\begin{array}{l}\text { Jorissen et al., } \\
1993\end{array}$ & this study \\
\hline MD99-2346 & 42.07 & 4.15 & Mediterranean & 2100 & Forams & Melki et al., 2009 & this study \\
\hline M40/4 82-2-SL & 42.31 & 3.77 & Mediterranean & 1070 & Forams & $\begin{array}{l}\text { Hayes, } \\
\text { unpublished data }\end{array}$ & this study \\
\hline V30-36 & 5.35 & -27.32 & North Atlantic & 4245 & Forams & Mix, 1986 & this study \\
\hline M35003-4 & 12.09 & -61.24 & North Atlantic & 1299 & Forams & Hüls et al., 2000 & this study \\
\hline KNR140-2_51GGC & 32.78 & -76.28 & North Atlantic & 1790 & Forams & $\begin{array}{l}\text { Rasmussen and } \\
\text { Thomson, } 2012\end{array}$ & this study \\
\hline MD95-2040 & 40.58 & -9.86 & North Atlantic & 2465 & Forams & $\begin{array}{l}\text { deAbreu et al., } \\
2003\end{array}$ & this study \\
\hline CH69-K09 & 41.76 & -47.35 & North Atlantic & 4100 & Forams & $\begin{array}{l}\text { Labeyrie et al., } \\
1999\end{array}$ & this study \\
\hline $\begin{array}{l}\text { BENGAL } \\
13078 \# 16\end{array}$ & 48.83 & -16.45 & North Atlantic & 4844 & Forams & Smart et al., 2009 & this study \\
\hline $\mathrm{CH} 77-02$ & 52.07 & -36.08 & North Atlantic & 3744 & Forams & $\begin{array}{l}\text { Marchal et al., } \\
2002\end{array}$ & this study \\
\hline NEAP17K & 54.68 & -28.35 & North Atlantic & 2734 & Forams & $\begin{array}{l}\text { Marchal et al., } \\
2002\end{array}$ & this study \\
\hline NA87-22 & 55.50 & -14.07 & North Atlantic & 2161 & Forams & Duplessy, 1992 & this study \\
\hline MD95-2015 & 58.76 & -25.96 & North Atlantic & 2630 & Forams & $\begin{array}{l}\text { Marchal et al., } \\
2002\end{array}$ & this study \\
\hline LINK14 & 61.80 & -6.30 & North Atlantic & 346 & Forams & $\begin{array}{l}\text { Rasmussen and } \\
\text { Thomson, } 2012\end{array}$ & this study \\
\hline MD99-2284 & 62.37 & -0.98 & North Atlantic & 1500 & Forams & $\begin{array}{l}\text { Risebrobakken et } \\
\text { al., } 2011\end{array}$ & this study \\
\hline
\end{tabular}




\begin{tabular}{|c|c|c|c|c|c|c|c|}
\hline MD95-2011 & 66.97 & 7.63 & North Atlantic & 1048 & Forams & Risebrobakken et & this study \\
\hline $23258-2$ & 75.00 & 13.97 & North Atlantic & 1768 & Forams & $\begin{array}{l}\text { Sarnthein et al., } \\
2003\end{array}$ & this study \\
\hline GIK18287-3 & 5.60 & 110.60 & Pacific & 598 & Forams & $\begin{array}{l}\text { Steinke et al., } \\
2001\end{array}$ & this study \\
\hline MD01-2390 & 6.63 & 113.40 & Pacific & 1545 & Forams & $\begin{array}{l}\text { Steinke et al., } \\
2008\end{array}$ & this study \\
\hline MD972151 & 8.73 & 109.87 & Pacific & 283 & Forams & Huang et al., 2002 & this study \\
\hline MD972142 & 12.69 & 119.47 & Pacific & 1557 & Forams & Yu et al., 2006 & this study \\
\hline MD012394 & 13.78 & 110.25 & Pacific & 2097 & Forams & Yu et al., 2006 & this study \\
\hline $17938-2$ & 19.78 & 117.53 & Pacific & 284 & Forams & Chen et al., 1999 & this study \\
\hline MD972148 & 19.80 & 117.54 & Pacific & 4872 & Forams & Chen et al., 1999 & this study \\
\hline MD97-2146 & 20.12 & 117.38 & Pacific & 172 & Forams & Lin et al., 2006 & this study \\
\hline RN96-PC1 & 24.97 & 122.93 & Pacific & 1676 & Forams & Ujiié et al., 2003 & this study \\
\hline 255 & 25.20 & 123.12 & Pacific & 1575 & Forams & Jian et al., 2000 & this study \\
\hline RN88-PC5 & 25.26 & 125.15 & Pacific & 2051 & Forams & Ujiié et al., 2003 & this study \\
\hline RN93-PC6 & 25.68 & 124.36 & Pacific & 1849 & Forams & Ujiié et al., 2003 & this study \\
\hline RN93-PC4 & 26.55 & 125.07 & Pacific & 1440 & Forams & Ujiié et al., 2003 & this study \\
\hline E017 & 26.57 & 126.03 & Pacific & 1826 & Forams & Xiang et al., 2007 & this study \\
\hline MD012404 & 26.65 & 125.81 & Pacific & 1397 & Forams & Chang et al., 2008 & this study \\
\hline MD98-2193 & 27.40 & 126.27 & Pacific & 1614 & Forams & Ujiié et al., 2003 & this study \\
\hline RN93-PC3 & 27.68 & 126.42 & Pacific & 1292 & Forams & Ujiié et al., 2003 & this study \\
\hline A7 & 27.82 & 126.97 & Pacific & 1264 & Forams & Xiang et al., 2007 & this study \\
\hline RN93-PC1 & 28.56 & 127.22 & Pacific & 1022 & Forams & Ujiié et al., 2003 & this study \\
\hline RN92-PC3 & 28.88 & 130.67 & Pacific & 2510 & Forams & Ujiié et al., 2003 & this study \\
\hline RN94-PC3 & 30.92 & 131.85 & Pacific & 1536 & Forams & Ujiié et al., 2003 & this study \\
\hline B-3GC & 31.48 & 128.52 & Pacific & 555 & Forams & Jian et al., 2000 & this study \\
\hline RN92-PC4 & 31.67 & 128.72 & Pacific & 710 & Forams & Ujiié et al., 2003 & this study \\
\hline
\end{tabular}




\begin{tabular}{|c|c|c|c|c|c|c|c|}
\hline RC24-16 & -5.04 & -10.19 & South Atlantic & 3543 & Forams & Mix, 1986 & this study \\
\hline GIK16773-1 & -0.97 & -9.44 & South Atlantic & 4662 & Forams & Mix, 1986 & this study \\
\hline MD97-2125 & -22.57 & 161.73 & Pacific & 1684 & $\mathrm{Mg} / \mathrm{Ca}$ & $\begin{array}{l}\text { Tachikawa et al., } \\
2009\end{array}$ & GHOST \\
\hline MD01-2378 & -13.08 & 121.79 & Indian Ocean & 1783 & $\mathrm{Mg} / \mathrm{Ca}$ & Xu et al., 2008 & GHOST \\
\hline MD98-2165 & -9.65 & 118.34 & Indian Ocean & 2100 & $\mathrm{Mg} / \mathrm{Ca}$ & Levi et al., 2007 & GHOST \\
\hline GeoB10038-4 & -5.93 & 103.25 & Indian Ocean & 1819 & $\mathrm{Mg} / \mathrm{Ca}$ & $\begin{array}{l}\text { Mohtadi et al., } \\
\text { 2010b }\end{array}$ & this study \\
\hline MD98-2162 & -4.69 & 117.90 & Indian Ocean & 1855 & $\mathrm{Mg} / \mathrm{Ca}$ & Visser et al., 2003 & GHOST \\
\hline GeoB10029-4 & -1.50 & 100.13 & Indian Ocean & 772 & $\mathrm{Mg} / \mathrm{Ca}$ & $\begin{array}{l}\text { Mohtadi et al., } \\
\text { 2010b }\end{array}$ & this study \\
\hline GeoB4905-4 & 2.50 & 9.39 & North Atlantic & 1328 & $\mathrm{Mg} / \mathrm{Ca}$ & $\begin{array}{l}\text { Weldeab et al., } \\
2007 b\end{array}$ & GHOST \\
\hline MD03-2707 & 2.50 & 9.40 & North Atlantic & 1295 & $\mathrm{Mg} / \mathrm{Ca}$ & $\begin{array}{l}\text { Weldeab et al., } \\
2007 a\end{array}$ & GHOST \\
\hline PL07-39PC & 10.70 & -65.94 & North Atlantic & 790 & $\mathrm{Mg} / \mathrm{Ca}$ & Lea et al., 2003 & GHOST \\
\hline VM28-122 & 11.57 & -78.42 & North Atlantic & 3623 & $\mathrm{Mg} / \mathrm{Ca}$ & $\begin{array}{l}\text { Schmidt et al., } \\
2004\end{array}$ & GHOST \\
\hline ODP999 & 12.75 & -78.73 & North Atlantic & 2827 & $\mathrm{Mg} / \mathrm{Ca}$ & $\begin{array}{l}\text { Schmidt et al., } \\
2004\end{array}$ & GHOST \\
\hline BOFS31K & 19.00 & -20.17 & North Atlantic & 3300 & $\mathrm{Mg} / \mathrm{Ca}$ & $\begin{array}{l}\text { Elderfield and } \\
\text { Ganssen, } 2000\end{array}$ & GHOST \\
\hline MD02-2575 & 29.02 & -87.12 & North Atlantic & 847 & $\mathrm{Mg} / \mathrm{Ca}$ & $\begin{array}{l}\text { Nürnberg et al., } \\
2008\end{array}$ & GHOST \\
\hline MD01-2334 & 37.80 & -10.17 & North Atlantic & 3166 & $\mathrm{Mg} / \mathrm{Ca}$ & $\begin{array}{l}\text { Skinner and } \\
\text { Elderfield, } 2005\end{array}$ & GHOST \\
\hline OCE326-GGC26 & 43.48 & -54.87 & North Atlantic & 3975 & $\mathrm{Mg} / \mathrm{Ca}$ & $\begin{array}{l}\text { Keigwin et al., } \\
2005\end{array}$ & GHOST \\
\hline MD99-2251 & 57.43 & -27.90 & North Atlantic & 2620 & $\mathrm{Mg} / \mathrm{Ca}$ & $\begin{array}{l}\text { Farmer et al., } \\
2011\end{array}$ & this study \\
\hline ODP984 & 61.00 & -25.00 & North Atlantic & 1648 & $\mathrm{Mg} / \mathrm{Ca}$ & Came et al., 2007 & this study \\
\hline RAPID-12-1K & 62.09 & -17.82 & North Atlantic & 1938 & $\mathrm{Mg} / \mathrm{Ca}$ & Thornalley et al., & GHOST \\
\hline
\end{tabular}




\begin{tabular}{|c|c|c|c|c|c|c|c|}
\hline & & & & & & 2009 & \\
\hline MD97-2120 & -45.53 & 174.93 & Pacific & 1210 & $\mathrm{Mg} / \mathrm{Ca}$ & $\begin{array}{l}\text { Pahnke et al., } \\
2003\end{array}$ & GHOST \\
\hline MD98-2170 & -10.59 & 125.39 & Pacific & 832 & $\mathrm{Mg} / \mathrm{Ca}$ & Stott et al., 2004 & GHOST \\
\hline MD98-2176 & -5.00 & 133.45 & Pacific & 2382 & $\mathrm{Mg} / \mathrm{Ca}$ & Stott et al., 2004 & GHOST \\
\hline V19-28 & -2.37 & -84.65 & Pacific & 2720 & $\mathrm{Mg} / \mathrm{Ca}$ & $\begin{array}{l}\text { Koutavas et al., } \\
2006\end{array}$ & GHOST \\
\hline V21-30 & -1.22 & -89.68 & Pacific & 617 & $\mathrm{Mg} / \mathrm{Ca}$ & $\begin{array}{l}\text { Koutavas et al., } \\
2002\end{array}$ & GHOST \\
\hline ODP1240 & 0.02 & -86.45 & Pacific & 2921 & $\mathrm{Mg} / \mathrm{Ca}$ & Pena et al., 2008 & GHOST \\
\hline TR163-22 & 0.52 & -92.40 & Pacific & 2830 & $\mathrm{Mg} / \mathrm{Ca}$ & Lea et al., 2006 & GHOST \\
\hline TR163-19 & 2.26 & -90.95 & Pacific & 2348 & $\mathrm{Mg} / \mathrm{Ca}$ & Lea et al., 2000 & GHOST \\
\hline MD98-2181 & 6.30 & 125.83 & Pacific & 2114 & $\mathrm{Mg} / \mathrm{Ca}$ & Stott et al., 2004 & GHOST \\
\hline $\begin{array}{l}\text { ODP1242/ME000 } \\
\text { 5A-43JC }\end{array}$ & 7.86 & -83.61 & Pacific & 1366 & $\mathrm{Mg} / \mathrm{Ca}$ & $\begin{array}{l}\text { Benway et al., } \\
2006\end{array}$ & GHOST \\
\hline MD97-2141 & 8.80 & 121.30 & Pacific & 3633 & $\mathrm{Mg} / \mathrm{Ca}$ & $\begin{array}{l}\text { Rosenthal et al., } \\
2003\end{array}$ & GHOST \\
\hline MD05-2904 & 19.45 & 116.25 & Pacific & 2066 & $\mathrm{Mg} / \mathrm{Ca}$ & $\begin{array}{l}\text { Steinke et al., } \\
2011\end{array}$ & this study \\
\hline ODP1145 & 19.58 & 117.63 & Pacific & 3175 & $\mathrm{Mg} / \mathrm{Ca}$ & $\begin{array}{l}\text { Oppo and Sun, } \\
2005\end{array}$ & GHOST \\
\hline A7 & 27.82 & 126.98 & Pacific & 1264 & $\mathrm{Mg} / \mathrm{Ca}$ & Sun et al., 2005 & GHOST \\
\hline KY07-04-PC1 & 31.63 & 128.95 & Pacific & 758 & $\mathrm{Mg} / \mathrm{Ca}$ & $\begin{array}{l}\text { Kubota et al., } \\
2010\end{array}$ & this study \\
\hline MD01-2416 & 51.00 & 168.00 & Pacific & 2317 & $\mathrm{Mg} / \mathrm{Ca}$ & $\begin{array}{l}\text { Sarnthein et al., } \\
2004\end{array}$ & GHOST \\
\hline ODP1084B & -25.51 & 13.28 & South Atlantic & 1992 & $\mathrm{Mg} / \mathrm{Ca}$ & $\begin{array}{l}\text { Farmer et al., } \\
2005\end{array}$ & GHOST \\
\hline GeoB3129/3911 & -4.61 & -36.64 & South Atlantic & 830 & $\mathrm{Mg} / \mathrm{Ca}$ & $\begin{array}{l}\text { Weldeab et al., } \\
2006\end{array}$ & GHOST \\
\hline
\end{tabular}


Table 2: Summary information for individual annual records

\begin{tabular}{|c|c|c|c|c|c|c|c|c|c|c|c|c|c|c|c|c|c|c|}
\hline \multicolumn{5}{|c|}{ ANNUAL } & \multicolumn{7}{|c|}{ 5.5-6.5 ka BP } & \multicolumn{7}{|c|}{$5.75-6.25 \mathrm{ka} \mathrm{BP}$} \\
\hline$\frac{0}{0}$ & $\begin{array}{l}z \\
+ \\
0 \\
1 \\
0 \\
0 \\
0 \\
0 \\
0 \\
0 \\
.0 \\
0 \\
0 \\
0\end{array}$ & 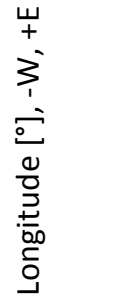 & 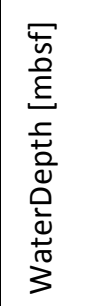 & 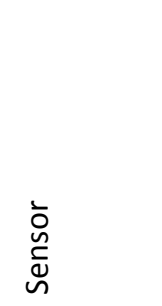 & 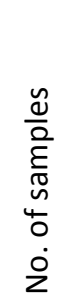 & 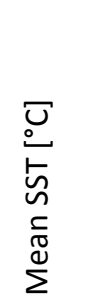 & 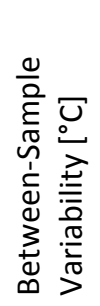 & 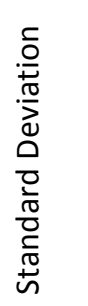 & 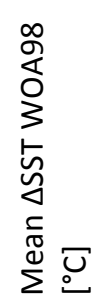 & 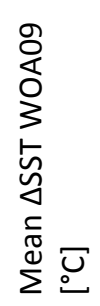 & 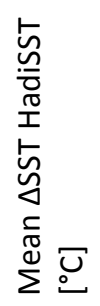 & 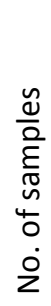 & 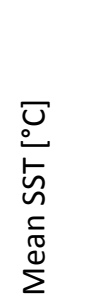 & 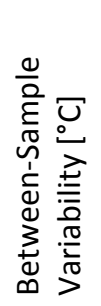 & 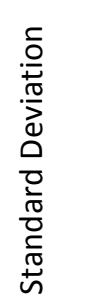 & 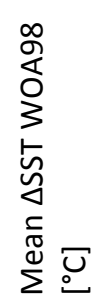 & 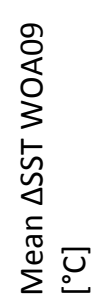 & 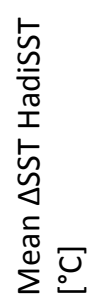 \\
\hline MD94-103 & -45.58 & 86.53 & 3560 & Alkenone & 4 & 9.8 & 1.30 & 0.54 & 0.56 & 0.53 & 0.79 & 3 & 9.57 & 0.60 & 0.32 & 0.33 & 0.30 & 0.56 \\
\hline MD97-2120 & -45.53 & 174.93 & 1210 & Alkenone & 7 & 10.52 & 0.59 & 0.21 & -0.47 & -0.50 & -0.57 & 3 & 10.63 & 0.30 & 0.16 & -0.36 & -0.39 & -0.45 \\
\hline MD97-2120 & -45.53 & 174.93 & 1210 & $\mathrm{Mg} / \mathrm{Ca}$ & 1 & - & - & - & - & - & - & 1 & - & - & - & - & - & - \\
\hline S0136-GC11 & -43.44 & 167.85 & 1556 & Alkenone & 3 & 15.86 & 0.38 & 0.20 & 1.35 & 1.34 & 1.70 & 1 & 15.79 & - & - & 1.28 & 1.27 & 1.63 \\
\hline TN057-21PC & -41.13 & 8.80 & 4981 & Alkenone & 6 & 18.27 & 2.27 & 0.72 & 6.78 & 7.18 & 6.87 & 3 & 18.34 & 0.19 & 0.11 & 6.85 & 7.25 & 6.94 \\
\hline ODP1233 & -41.01 & -74.45 & 838 & Alkenone & 3 & 14.94 & 0.14 & 0.07 & 1.66 & 1.92 & 2.02 & 2 & 14.98 & 0.04 & 0.03 & 1.69 & 1.96 & 2.06 \\
\hline GeoB3313-1 & -41.00 & -74.45 & 852 & Alkenone & 10 & 15.75 & 0.82 & 0.26 & 2.46 & 2.73 & 2.52 & 4 & 15.62 & 0.26 & 0.11 & 2.33 & 2.60 & 2.40 \\
\hline MD97-2121 & -40.38 & 177.99 & 3014 & Alkenone & 15 & 17.45 & 1.52 & 0.40 & 0.81 & 0.85 & 2.35 & 8 & 17.34 & 1.42 & 0.50 & 0.70 & 0.74 & 2.24 \\
\hline MD03-2611 & -36.73 & 136.55 & 2420 & Alkenone & 3 & 19.62 & 0.28 & 0.14 & 3.45 & 3.26 & 3.73 & 1 & 19.62 & 0.00 & - & 3.45 & 3.26 & 3.73 \\
\hline GIK17748-2 & -32.75 & -72.03 & 2545 & Alkenone & 2 & 18.70 & 0.40 & 0.28 & 3.78 & 3.46 & 3.60 & 1 & 18.50 & 0.00 & - & 3.58 & 3.26 & 3.40 \\
\hline $\begin{array}{l}\text { GIK17748-2 } \\
\text { (SO80-4) }\end{array}$ & -32.75 & -72.03 & 2545 & Foram & 2 & 13.12 & 1.22 & 0.87 & -1.79 & -2.12 & -1.98 & 1 & 12.51 & 0.00 & - & -2.41 & -2.73 & -2.59 \\
\hline GeoB7139-2 & -30.20 & -71.98 & 3270 & Alkenone & 2 & 18.72 & 0.15 & 0.11 & 3.24 & 3.19 & 3.21 & 1 & 18.79 & 0.00 & - & 3.32 & 3.26 & 3.28 \\
\hline GeoB7139-2 & -30.20 & -71.98 & 3269 & Foram & 2 & 12.52 & 0.04 & 0.03 & -2.95 & -3.01 & -2.99 & 1 & 12.54 & 0.00 & - & -2.93 & -2.99 & -2.97 \\
\hline ODP1084B & -25.51 & 13.28 & 1992 & $\mathrm{Mg} / \mathrm{Ca}$ & 8 & - & - & - & - & - & - & 3 & - & - & - & - & - & - \\
\hline GeoB7112-5 & -24.03 & -70.82 & 2507 & Foram & 3 & 12.43 & 0.58 & 0.30 & -5.65 & -5.71 & -5.24 & 2 & 12.31 & 0.45 & 0.32 & -5.77 & -5.83 & -5.36 \\
\hline GeoB1710-3 & -23.43 & 11.69 & 1045 & Alkenone & 1 & 19.50 & - & - & 2.14 & 1.77 & 0.99 & 1 & 19.50 & 0.00 & - & 2.14 & 1.77 & 0.99 \\
\hline GeoB1711-4 & -23.31 & 12.37 & 1967 & Alkenone & 1 & 19.70 & - & - & 2.98 & 2.88 & 1.94 & 1 & 19.70 & 0.00 & - & 2.97 & 2.88 & 1.94 \\
\hline GeoB1712-4 & -23.26 & 12.81 & 998 & Alkenone & 2 & 19.30 & 0.20 & 0.14 & 3.01 & 2.48 & 1.54 & 2 & 19.30 & 0.20 & 0.14 & 3.01 & 2.48 & 1.54 \\
\hline MD97-2125 & -22.57 & 161.73 & 1684 & $\mathrm{Mg} / \mathrm{Ca}$ & 2 & - & - & - & - & - & - & 0 & - & - & - & - & - & - \\
\hline
\end{tabular}




\begin{tabular}{|c|c|c|c|c|c|c|c|c|c|c|c|c|c|c|c|c|c|c|}
\hline MD79257 & -20.40 & 36.33 & 1262 & Alkenone & 2 & 27.20 & 0.20 & 0.14 & 1.32 & 0.98 & 1.23 & 1 & 27.30 & 0.00 & - & 1.41 & 1.08 & 1.33 \\
\hline GeoB1023-5 & -17.16 & 11.01 & 1978 & Alkenone & 3 & 22.08 & 0.18 & 0.10 & 3.28 & 3.32 & 2.80 & 1 & 22.15 & 0.00 & - & 3.35 & 3.39 & 2.87 \\
\hline MD01-2378 & -13.08 & 121.79 & 1783 & $\mathrm{Mg} / \mathrm{Ca}$ & 8 & - & - & - & - & - & - & 4 & - & - & - & - & - & - \\
\hline ODP1078C & -11.92 & 13.40 & 426 & Alkenone & 3 & 24.43 & 0.20 & 0.12 & 0.07 & -0.81 & -0.06 & 1 & 24.50 & 0.00 & - & 0.13 & -0.74 & 0.01 \\
\hline MD05-2928 & -11.29 & 148.86 & 2250 & Alkenone & 3 & 28.19 & 0.08 & 0.04 & 0.75 & 0.72 & 0.97 & 1 & 28.16 & 0.00 & - & 0.71 & 0.69 & 0.94 \\
\hline MD98-2170 & -10.59 & 125.39 & 832 & $\mathrm{Mg} / \mathrm{Ca}$ & 4 & - & - & - & - & - & - & 1 & - & - & - & - & - & - \\
\hline MD98-2165 & -9.65 & 118.34 & 2100 & $\mathrm{Mg} / \mathrm{Ca}$ & 5 & - & - & - & - & - & - & 2 & - & - & - & - & - & - \\
\hline SO139-74KL & -6.54 & 103.83 & 1690 & Alkenone & 24 & 27.48 & 0.79 & 0.17 & -0.71 & -0.73 & -0.78 & 12 & 27.46 & 0.77 & 0.19 & -0.73 & -0.76 & -0.80 \\
\hline GeoB10038-4 & -5.94 & 103.25 & 1819 & Alkenone & 2 & 27.40 & 0.00 & 0.00 & -0.75 & -0.76 & -0.90 & 1 & 27.40 & 0.00 & - & -0.75 & -0.76 & -0.90 \\
\hline GeoB10038-4 & -5.93 & 103.25 & 1819 & $\mathrm{Mg} / \mathrm{Ca}$ & 1 & - & - & - & - & - & - & 1 & - & - & - & - & - & - \\
\hline GeoB6518-1 & -5.59 & 11.22 & 962 & Alkenone & 6 & 24.50 & 0.50 & 0.21 & 0.12 & -1.11 & -0.96 & 2 & 24.65 & 0.10 & 0.07 & 0.27 & -0.96 & -0.81 \\
\hline RC24-16 & -5.04 & -10.19 & 3543 & Foram & 2 & 25.92 & 0.42 & 0.30 & 0.41 & 0.28 & 0.25 & 0 & - & - & - & - & - & - \\
\hline MD98-2176 & -5.00 & 133.45 & 2382 & $\mathrm{Mg} / \mathrm{Ca}$ & 24 & - & - & - & - & - & - & 11 & - & - & - & - & - & - \\
\hline MD98-2162 & -4.69 & 117.90 & 1855 & $\mathrm{Mg} / \mathrm{Ca}$ & 2 & - & - & - & - & - & - & 1 & - & - & - & - & - & - \\
\hline GeoB 3129-1 & -4.61 & -36.64 & 830 & Alkenone & 3 & 28.12 & 0.15 & 0.08 & 0.82 & 0.92 & 0.82 & 1 & 28.15 & 0.00 & - & 0.85 & 0.95 & 0.85 \\
\hline $\begin{array}{l}\text { GeoB3129/ } \\
3911\end{array}$ & -4.61 & -36.64 & 830 & $\mathrm{Mg} / \mathrm{Ca}$ & 3 & - & - & - & - & - & - & 1 & - & - & - & - & - & - \\
\hline GeoB3910-2 & -4.02 & -36.35 & 2362 & Alkenone & 2 & 27.05 & 0.70 & 0.49 & -0.26 & -0.15 & -0.25 & 1 & 27.40 & 0.00 & - & 0.09 & 0.20 & 0.10 \\
\hline V19-30 & -3.38 & -83.52 & 3091 & Alkenone & 1 & 22.49 & - & - & 0.56 & 0.37 & -0.33 & 1 & 22.49 & 0.00 & - & 0.56 & 0.37 & -0.33 \\
\hline V19-28 & -2.51 & -84.65 & 2720 & Alkenone & 1 & 23.72 & - & - & 1.08 & 0.87 & 0.41 & 1 & 23.72 & 0.00 & - & 1.08 & 0.87 & 0.41 \\
\hline V19-28 & -2.37 & -84.65 & 2720 & $\mathrm{Mg} / \mathrm{Ca}$ & 1 & - & - & - & - & - & - & 1 & - & - & - & - & - & - \\
\hline GeoB10029-4 & -1.50 & 100.13 & 772 & $\mathrm{Mg} / \mathrm{Ca}$ & 2 & - & - & - & - & - & - & 2 & - & - & - & - & - & - \\
\hline V21-30 & -1.22 & -89.68 & 617 & Alkenone & 3 & 24.70 & 0.41 & 0.20 & 1.91 & 1.29 & 0.96 & 1 & 24.90 & 0.00 & - & 2.10 & 1.49 & 1.16 \\
\hline V21-30 & -1.22 & -89.68 & 617 & $\mathrm{Mg} / \mathrm{Ca}$ & 5 & - & - & - & - & - & - & 1 & - & - & - & - & - & - \\
\hline GIK16773-1 & -0.97 & -9.44 & 4662 & Foram & 2 & 25.81 & 0.01 & 0.01 & 0.20 & -0.07 & 0.14 & 1 & 25.81 & 0.00 & - & 0.19 & -0.08 & 0.14 \\
\hline V19-27 & -0.47 & -82.67 & 1373 & Alkenone & 1 & 25.21 & - & - & 1.21 & 0.69 & 0.51 & 1 & 25.21 & 0.00 & - & 1.21 & 0.69 & 0.51 \\
\hline ODP1240 & 0.02 & -86.45 & 2921 & $\mathrm{Mg} / \mathrm{Ca}$ & 8 & - & - & - & - & - & - & 4 & - & - & - & - & - & - \\
\hline TR163-22 & 0.52 & -92.40 & 2830 & $\mathrm{Mg} / \mathrm{Ca}$ & 3 & - & - & - & - & - & - & 2 & - & - & - & - & - & - \\
\hline $\begin{array}{l}\text { ME0005A- } \\
24 J C\end{array}$ & 1.50 & -86.49 & 2941 & Alkenone & 3 & 24.27 & 0.64 & 0.35 & -1.34 & -1.53 & -1.44 & 2 & 24.07 & 0.08 & 0.06 & -1.54 & -1.73 & -1.64 \\
\hline TR163-19 & 2.26 & -90.95 & 2348 & $\mathrm{Mg} / \mathrm{Ca}$ & 1 & - & - & - & - & - & - & 0 & - & - & - & - & - & - \\
\hline GeoB4905-4 & 2.50 & 9.39 & 1328 & Alkenone & 6 & 25.07 & 0.35 & 0.13 & -2.49 & -2.77 & -2.46 & 3 & 25.11 & 0.16 & 0.12 & -2.45 & -2.73 & -2.43 \\
\hline
\end{tabular}




\begin{tabular}{|c|c|c|c|c|c|c|c|c|c|c|c|c|c|c|c|c|c|c|}
\hline GeoB4905-4 & 2.50 & 9.39 & 1328 & $\mathrm{Mg} / \mathrm{Ca}$ & 9 & - & - & - & - & - & - & 6 & - & - & - & - & - & - \\
\hline MD03-2707 & 2.50 & 9.40 & 1295 & $\mathrm{Mg} / \mathrm{Ca}$ & 24 & - & - & - & - & - & - & 12 & - & - & - & - & - & - \\
\hline MD85674 & 3.18 & 50.43 & 4875 & Alkenone & 2 & 27.05 & 0.50 & 0.35 & -0.07 & 0.02 & 0.18 & 0 & - & - & - & - & - & - \\
\hline $\begin{array}{l}\text { KNR176- } \\
\text { JPC32 }\end{array}$ & 4.85 & -77.96 & 2200 & Alkenone & 4 & 26.04 & 0.24 & 0.16 & -0.33 & -0.67 & -0.98 & 2 & 26.04 & 0.09 & 0.06 & -0.34 & -0.68 & -0.98 \\
\hline V30-36 & 5.35 & -27.32 & 4245 & Foram & 1 & 27.38 & - & - & -0.09 & -0.03 & 0.28 & 1 & 27.38 & 0.00 & - & -0.09 & -0.02 & 0.29 \\
\hline GIK18287-3 & 5.60 & 110.60 & 598 & Foram & 3 & 27.72 & 1.28 & 0.67 & -0.55 & -0.66 & -0.41 & 1 & 28.24 & 0.00 & - & -0.03 & -0.14 & 0.11 \\
\hline $18287-3$ & 5.65 & 110.65 & 598 & Alkenone & 3 & 28.06 & 0.16 & 0.08 & -0.21 & -0.32 & -0.07 & 1 & 28.15 & 0.00 & - & -0.12 & -0.23 & 0.02 \\
\hline GIK18287-3 & 5.98 & 110.65 & 598 & Alkenone & 3 & 28.07 & 0.16 & 0.08 & -0.18 & -0.31 & -0.06 & 1 & 28.15 & 0.00 & - & -0.10 & -0.23 & 0.02 \\
\hline 17964 & 6.16 & 112.21 & 1556 & Alkenone & 2 & 27.70 & 0.03 & 0.02 & -0.66 & -0.72 & -0.49 & 0 & - & - & - & - & - & - \\
\hline MD98-2181 & 6.30 & 125.83 & 2114 & $\mathrm{Mg} / \mathrm{Ca}$ & 22 & - & - & - & - & - & - & 12 & - & - & - & - & - & - \\
\hline MD01-2390 & 6.63 & 113.40 & 1545 & Foram & 2 & 28.55 & 0.01 & 0.01 & 0.14 & 0.09 & 0.28 & 1 & 28.54 & 0.00 & - & 0.13 & 0.08 & 0.28 \\
\hline MD01-2390 & 6.64 & 113.41 & 1545 & $\mathrm{Mg} / \mathrm{Ca}$ & 3 & - & - & - & - & - & - & 1 & - & - & - & - & - & - \\
\hline $\begin{array}{l}\text { ODP1242/ME } \\
0005 A-43 J C\end{array}$ & 7.86 & -83.61 & 1366 & $\mathrm{Mg} / \mathrm{Ca}$ & 9 & - & - & - & - & - & - & 4 & - & - & - & - & - & - \\
\hline MD02-2529 & 8.21 & -84.12 & 1619 & Alkenone & 3 & 27.40 & 0.79 & 0.43 & -0.81 & -1.09 & -0.65 & 2 & 27.55 & 0.68 & 0.48 & -0.66 & -0.94 & -0.50 \\
\hline MD97-2151 & 8.73 & 109.87 & 1598 & Alkenone & 10 & 27.42 & 1.57 & 0.40 & -0.52 & -0.56 & -0.32 & 5 & 27.32 & 1.10 & 0.45 & -0.62 & -0.66 & -0.43 \\
\hline MD972151 & 8.73 & 109.87 & 283 & Foram & 3 & 27.70 & 0.69 & 0.39 & -0.24 & -0.28 & -0.04 & 1 & 27.94 & 0.00 & - & 0.00 & -0.04 & 0.20 \\
\hline MD97-2141 & 8.80 & 121.30 & 3633 & $\mathrm{Mg} / \mathrm{Ca}$ & 15 & - & - & - & - & - & - & 7 & - & - & - & - & - & - \\
\hline $18252-3$ & 9.23 & 109.38 & 1273 & Alkenone & 3 & 27.21 & 0.32 & 0.16 & -0.57 & -0.63 & -0.39 & 1 & 27.25 & 0.00 & - & -0.53 & -0.59 & -0.35 \\
\hline MD01-2392 & 9.85 & 110.21 & 1966 & Alkenone & 1 & 27.45 & - & - & -0.39 & -0.55 & -0.26 & 1 & 27.45 & 0.00 & - & -0.39 & -0.55 & -0.26 \\
\hline MD77194 & 10.47 & 75.23 & 1222 & Alkenone & 2 & 27.15 & 0.10 & 0.07 & -1.18 & -1.45 & -1.23 & 1 & 27.10 & 0.00 & - & -1.23 & -1.50 & -1.28 \\
\hline PL07-39PC & 10.70 & -65.94 & 790 & $\mathrm{Mg} / \mathrm{Ca}$ & 4 & - & - & - & - & - & - & 2 & - & - & - & - & - & - \\
\hline ODP 1002C & 10.71 & -65.17 & 893 & Alkenone & 1 & 25.80 & - & - & -0.82 & -1.90 & -1.12 & 1 & 25.80 & 0.00 & - & -0.82 & -1.90 & -1.12 \\
\hline TY93-905 & 11.07 & 51.95 & 1567 & Alkenone & 7 & 25.23 & 0.45 & 0.18 & -0.82 & -0.92 & -1.75 & 4 & 25.17 & 0.33 & 0.16 & -0.88 & -0.99 & -1.82 \\
\hline VM28-122 & 11.57 & -78.42 & 3623 & $\mathrm{Mg} / \mathrm{Ca}$ & 1 & - & - & - & - & - & - & 1 & - & - & - & - & - & - \\
\hline M35003-4 & 12.08 & -61.25 & 1299 & Alkenone & 4 & 27.85 & 0.50 & 0.21 & 0.43 & 0.36 & 0.29 & 2 & 27.95 & 0.30 & 0.21 & 0.53 & 0.46 & 0.39 \\
\hline M35003-4 & 12.09 & -61.24 & 1299 & Foram & 3 & 27.64 & 0.05 & 0.03 & 0.22 & 0.15 & 0.08 & 2 & 27.62 & 0.02 & 0.01 & 0.20 & 0.13 & 0.06 \\
\hline MD972142 & 12.69 & 119.47 & 1557 & Foram & 4 & 27.92 & 0.78 & 0.33 & -0.57 & -0.48 & -0.37 & 3 & 27.89 & 0.78 & 0.39 & -0.60 & -0.51 & -0.40 \\
\hline ODP999 & 12.75 & -78.73 & 2827 & $\mathrm{Mg} / \mathrm{Ca}$ & 1 & - & - & - & - & - & - & 1 & - & - & - & - & - & - \\
\hline MD012394 & 13.78 & 110.25 & 2097 & Foram & 5 & 26.95 & 0.87 & 0.39 & -0.41 & -0.66 & -0.11 & 2 & 27.09 & 0.43 & 0.30 & -0.26 & -0.52 & 0.03 \\
\hline $74 \mathrm{KL}$ & 14.32 & 57.35 & 3212 & Alkenone & 2 & 27.23 & 0.03 & 0.02 & 0.60 & 0.59 & 0.74 & 1 & 27.21 & 0.00 & - & 0.58 & 0.57 & 0.72 \\
\hline
\end{tabular}




\begin{tabular}{|c|c|c|c|c|c|c|c|c|c|c|c|c|c|c|c|c|c|c|}
\hline SCS90-36 & 18.00 & 111.49 & 2050 & Alkenone & 2 & 24.91 & 2.06 & 1.46 & -1.87 & -2.00 & -1.74 & 1 & 25.94 & 0.00 & - & -0.84 & -0.97 & -0.71 \\
\hline BOFS 31K & 19.00 & -20.17 & 3300 & Alkenone & 1 & 21.30 & - & - & -0.95 & -1.07 & -1.48 & 0 & - & - & - & - & - & - \\
\hline BOFS31K & 19.00 & -20.17 & 3300 & $\mathrm{Mg} / \mathrm{Ca}$ & 1 & - & - & - & - & - & - & 0 & - & - & - & - & - & - \\
\hline MD05-2904 & 19.45 & 116.25 & 2066 & $\mathrm{Mg} / \mathrm{Ca}$ & 4 & - & - & - & - & - & - & 2 & - & - & - & - & - & - \\
\hline ODP1145 & 19.58 & 117.63 & 3175 & $\mathrm{Mg} / \mathrm{Ca}$ & 1 & - & - & - & - & - & - & 1 & - & - & - & - & - & - \\
\hline $17938-2$ & 19.78 & 117.53 & 284 & Foram & 1 & 25.54 & - & - & -0.99 & -1.25 & -0.91 & 0 & - & - & - & - & - & - \\
\hline MD972148 & 19.80 & 117.54 & 4872 & Foram & 3 & 25.59 & 0.63 & 0.45 & -0.93 & -1.20 & -0.86 & 1 & 25.25 & 0.00 & - & -1.28 & -1.53 & -1.20 \\
\hline S093-126KL & 19.97 & 90.03 & 1253 & Alkenone & 2 & 27.80 & 1.20 & 0.85 & -0.02 & 0.06 & 0.01 & 0 & - & - & - & - & - & - \\
\hline $17940-2$ & 20.12 & 117.38 & 1727 & Alkenone & 8 & 26.60 & 0.39 & 0.12 & 0.23 & 0.18 & 0.57 & 8 & 26.60 & 0.39 & 0.12 & 0.22 & 0.19 & 0.58 \\
\hline MD97-2146 & 20.12 & 117.38 & 172 & Foram & 21 & 26.32 & 1.96 & 0.45 & -0.05 & -0.10 & 0.29 & 10 & 26.25 & 1.82 & 0.52 & -0.12 & -0.17 & 0.22 \\
\hline GeoB7926-2 & 20.22 & -18.45 & 2500 & Alkenone & 2 & 21.20 & 0.60 & 0.42 & 0.78 & 0.61 & -0.58 & 1 & 20.90 & 0.00 & - & 0.48 & 0.31 & -0.88 \\
\hline ODP658C & 20.75 & -18.58 & 2262 & Alkenone & 8 & 21.18 & 0.70 & 0.28 & 0.89 & 0.59 & -0.60 & 4 & 21.35 & 0.40 & 0.19 & 1.06 & 0.76 & -0.43 \\
\hline PC17 & 21.36 & -158.19 & 503 & Alkenone & 3 & 25.53 & 0.10 & 0.06 & 0.33 & 0.05 & 0.19 & 1 & 25.50 & 0.00 & - & 0.30 & 0.02 & 0.16 \\
\hline LAPAZ21P & 22.99 & -109.47 & 624 & Alkenone & 1 & 24.70 & - & - & 0.39 & 0.03 & 0.63 & 1 & 24.70 & 0.00 & - & 0.39 & 0.03 & 0.63 \\
\hline SO90-93KL & 23.58 & 64.22 & 1802 & Alkenone & 3 & 27.55 & 0.30 & 0.16 & 0.97 & 0.82 & 0.96 & 2 & 27.49 & 0.25 & 0.18 & 0.91 & 0.75 & 0.90 \\
\hline RN96-PC1 & 24.97 & 122.93 & 1676 & Foram & 4 & 25.85 & 1.41 & 0.61 & 0.18 & -0.45 & 1.39 & 1 & 26.59 & 0.00 & - & 0.92 & 0.28 & 2.12 \\
\hline 255 & 25.20 & 123.12 & 1575 & Foram & 5 & 26.75 & 1.34 & 0.50 & 1.28 & 1.51 & 2.66 & 3 & 27.06 & 0.60 & 0.35 & 1.59 & 1.83 & 2.97 \\
\hline RN88-PC5 & 25.26 & 125.15 & 2051 & Foram & 2 & 25.64 & 0.23 & 0.16 & 0.28 & 0.32 & 0.73 & 2 & 25.64 & 0.23 & 0.16 & 0.27 & 0.32 & 0.74 \\
\hline RN93-PC6 & 25.68 & 124.36 & 1849 & Foram & 2 & 25.47 & 0.07 & 0.05 & 0.45 & 0.23 & 0.84 & 0 & - & - & - & - & - & - \\
\hline RN93-PC4 & 26.55 & 125.07 & 1440 & Foram & 2 & 25.81 & 0.28 & 0.20 & 1.35 & 1.13 & 1.71 & 1 & 25.95 & 0.00 & - & 1.50 & 1.27 & 1.85 \\
\hline E017 & 26.57 & 126.03 & 1826 & Foram & 3 & 26.36 & 0.73 & 0.42 & 1.67 & 1.45 & 2.00 & 1 & 26.61 & 0.00 & - & 1.92 & 1.70 & 2.25 \\
\hline MD012404 & 26.65 & 125.81 & 1397 & Foram & 9 & 25.90 & 1.42 & 0.46 & 1.33 & 1.22 & 1.80 & 4 & 26.01 & 1.42 & 0.64 & 1.43 & 1.33 & 1.91 \\
\hline MD98-2193 & 27.40 & 126.27 & 1614 & Foram & 1 & 25.87 & - & - & 1.72 & 1.61 & 2.36 & 0 & - & - & - & - & - & - \\
\hline RN93-PC3 & 27.68 & 126.42 & 1292 & Foram & 1 & 25.84 & - & - & 1.87 & 1.58 & 2.33 & 1 & 25.84 & 0.00 & - & 1.86 & 1.58 & 2.33 \\
\hline GeoB5844-2 & 27.71 & 34.68 & 963 & Alkenone & 5 & 26.26 & 0.60 & 0.25 & 1.00 & 0.99 & 1.77 & 3 & 26.30 & 0.30 & 0.17 & 1.04 & 1.03 & 1.81 \\
\hline A7 & 27.82 & 126.97 & 1264 & Foram & 7 & 26.24 & 0.84 & 0.35 & 2.18 & 1.98 & 2.73 & 4 & 26.43 & 0.84 & 0.36 & 2.36 & 2.17 & 2.92 \\
\hline A7 & 27.82 & 126.98 & 1264 & $\mathrm{Mg} / \mathrm{Ca}$ & 7 & - & - & - & - & - & - & 4 & - & - & - & - & - & - \\
\hline RN93-PC1 & 28.56 & 127.22 & 1022 & Foram & 1 & 25.14 & - & - & 1.53 & 1.21 & 1.96 & 1 & 25.14 & 0.00 & - & 1.54 & 1.21 & 1.95 \\
\hline RN92-PC3 & 28.88 & 130.67 & 2510 & Foram & 2 & 24.03 & 1.14 & 0.80 & -0.17 & -0.37 & 0.02 & 0 & - & - & - & - & - & - \\
\hline MD02-2575 & 29.02 & -87.12 & 847 & $\mathrm{Mg} / \mathrm{Ca}$ & 5 & - & - & - & - & - & - & 2 & - & - & - & - & - & - \\
\hline GeoB6007 & 30.85 & -10.27 & 583 & Alkenone & 33 & 20.08 & 0.80 & 0.24 & 1.67 & 1.58 & 0.72 & 17 & 20.07 & 0.60 & 0.18 & 1.67 & 1.57 & 0.71 \\
\hline RN94-PC3 & 30.92 & 131.85 & 1536 & Foram & 2 & 24.74 & 1.26 & 0.89 & 0.98 & 0.76 & 1.65 & 2 & 24.74 & 1.26 & 0.89 & 0.98 & 0.76 & 1.65 \\
\hline
\end{tabular}




\begin{tabular}{|c|c|c|c|c|c|c|c|c|c|c|c|c|c|c|c|c|c|c|}
\hline ST.19 & 31.10 & 138.67 & 3336 & Alkenone & 1 & 22.21 & - & - & -0.55 & -0.57 & -0.36 & 0 & - & - & - & - & - & - \\
\hline$B-3 G C$ & 31.48 & 128.52 & 555 & Foram & 6 & 23.93 & 0.73 & 0.26 & 1.95 & 1.72 & 2.76 & 3 & 23.85 & 0.49 & 0.25 & 1.86 & 1.64 & 2.69 \\
\hline KY07-04-PC1 & 31.63 & 128.95 & 758 & $\mathrm{Mg} / \mathrm{Ca}$ & 12 & - & - & - & - & - & - & 7 & - & - & - & - & - & - \\
\hline RN92-PC4 & 31.67 & 128.72 & 710 & Foram & 3 & 23.46 & 0.97 & 0.54 & 1.46 & 1.25 & 2.29 & 1 & 23.75 & 0.00 & - & 1.75 & 1.54 & 2.59 \\
\hline ODP1012B & 32.28 & -118.38 & 1772 & Alkenone & 1 & 16.10 & - & - & -0.13 & -0.33 & -0.12 & 1 & 16.10 & 0.00 & - & -0.13 & -0.33 & -0.12 \\
\hline ST.14 & 32.67 & 138.46 & 3252 & Alkenone & 1 & 22.54 & - & - & 0.33 & 0.14 & 0.40 & 1 & 22.54 & 0.00 & - & 0.33 & 0.14 & 0.40 \\
\hline $\begin{array}{l}\text { KNR140-2 } \\
51 G G C\end{array}$ & 32.78 & -76.28 & 1790 & Foram & 3 & 27.16 & 0.57 & 0.29 & 2.59 & 2.41 & 2.25 & 1 & 27.43 & 0.00 & - & 2.86 & 2.68 & 2.52 \\
\hline ODP969E & 33.84 & 24.88 & 2201 & Alkenone & 3 & 20.03 & 2.60 & 1.45 & -0.25 & -0.40 & -0.40 & 2 & 20.40 & 2.60 & 1.84 & 0.12 & -0.03 & -0.03 \\
\hline ODP 969 & 33.84 & 24.88 & 2200 & Foram & 2 & 20.46 & 0.21 & 0.15 & 0.18 & 0.03 & 0.03 & 1 & 20.57 & 0.00 & - & 0.28 & 0.14 & 0.14 \\
\hline ODP967D & 34.07 & 32.73 & 2551 & Alkenone & 3 & 20.61 & 1.64 & 0.92 & -0.89 & -0.32 & -0.97 & 1 & 20.12 & 0.00 & - & -1.38 & -0.81 & -1.46 \\
\hline ODP1017E & 34.54 & -121.11 & 955 & Alkenone & 2 & 15.12 & 0.65 & 0.46 & 0.88 & 0.67 & 0.24 & 1 & 15.44 & 0.00 & - & 1.21 & 0.99 & 0.56 \\
\hline SSDP-102 & 34.95 & 128.88 & 40 & Alkenone & 16 & 21.64 & 1.10 & 0.38 & 2.85 & 2.49 & 2.79 & 10 & 21.76 & 0.80 & 0.29 & 2.96 & 2.61 & 2.91 \\
\hline $\begin{array}{l}\text { TTR17- } \\
\text { MS419G }\end{array}$ & 35.45 & -4.62 & 410 & Foram & 1 & 13.77 & - & - & -4.05 & -4.43 & -4.39 & 1 & 13.77 & 0.00 & - & -4.05 & -4.44 & -4.39 \\
\hline KS310 & 35.55 & -1.57 & 1900 & Foram & 1 & 18.29 & - & - & 0.00 & -0.40 & -0.16 & 0 & - & - & - & - & - & - \\
\hline MD81-LC21 & 35.66 & 26.58 & 1522 & Foram & 5 & 19.91 & 0.39 & 0.15 & 0.08 & -0.01 & -0.24 & 3 & 19.82 & 0.10 & 0.05 & 0.00 & -0.09 & -0.32 \\
\hline ODP 973 & 35.78 & 18.95 & 3695 & Foram & 2 & 20.06 & 1.55 & 1.10 & 0.05 & -0.21 & -0.16 & 1 & 19.28 & 0.00 & - & -0.73 & -0.99 & -0.93 \\
\hline ODP977 & 36.03 & -1.96 & 1984 & Alkenone & 1 & 18.72 & - & - & 0.41 & -0.07 & 0.21 & 1 & 18.72 & 0.00 & - & 0.41 & -0.07 & 0.21 \\
\hline MD95-2043 & 36.14 & -2.62 & 1841 & Alkenone & 9 & 19.03 & 1.34 & 0.50 & 0.84 & 0.24 & 0.78 & 4 & 19.25 & 0.56 & 0.32 & 1.06 & 0.46 & 1.00 \\
\hline MD 95-2043 & 36.14 & -2.62 & 1841 & Foram & 4 & 18.21 & 0.17 & 0.08 & 0.02 & -0.58 & -0.04 & 2 & 18.16 & 0.00 & 0.00 & -0.03 & -0.63 & -0.09 \\
\hline MD95-2043 & 36.14 & -2.62 & 1841 & Dinocyst & 5 & 17.08 & 2.36 & 0.86 & -1.11 & -1.72 & -1.18 & 2 & 16.54 & 0.84 & 0.59 & -1.65 & -2.25 & -1.71 \\
\hline ODP 977 & 36.19 & -1.57 & 1984 & Foram & 2 & 17.60 & 2.04 & 1.44 & -0.76 & -1.19 & -0.91 & 1 & 18.62 & 0.00 & - & 0.26 & -0.17 & 0.11 \\
\hline $\begin{array}{l}\text { ODP Leg } 161 \\
\text { Site } 976 \text { Hole } \\
\text { C }\end{array}$ & 36.21 & -4.31 & 1108 & Dinocyst & 2 & 17.82 & 0.00 & 0.00 & 0.00 & -0.67 & -0.34 & 1 & 17.82 & 0.00 & - & 0.00 & -0.67 & -0.34 \\
\hline ODP 964 & 36.26 & 17.74 & 3657 & Foram & 1 & 19.87 & - & - & 0.00 & -0.11 & -0.03 & 1 & 19.87 & 0.00 & - & 0.00 & -0.12 & -0.03 \\
\hline M39-008 & 36.38 & -7.08 & 576 & Alkenone & 2 & 20.62 & 0.29 & 0.21 & 2.32 & 2.13 & 1.95 & 1 & 20.48 & 0.00 & - & 2.18 & 1.99 & 1.81 \\
\hline GeoB5901-2 & 36.38 & -7.07 & 574 & Alkenone & 29 & 19.86 & 0.74 & 0.17 & 1.55 & 1.37 & 1.19 & 14 & 19.98 & 0.47 & 0.13 & 1.67 & 1.50 & 1.31 \\
\hline RL11 & 36.75 & 17.72 & 3376 & Alkenone & 2 & 23.00 & 1.20 & 0.85 & 3.30 & 3.02 & 3.10 & 1 & 23.60 & 0.00 & - & 3.90 & 3.62 & 3.70 \\
\hline $\begin{array}{l}\text { CH07-98- } \\
\text { GGC19 }\end{array}$ & 36.87 & -74.57 & 1049 & Alkenone & 13 & 17.75 & 0.59 & 0.16 & -0.40 & -1.47 & -0.62 & 6 & 17.76 & 0.32 & 0.11 & -0.39 & -1.46 & -0.61 \\
\hline
\end{tabular}




\begin{tabular}{|c|c|c|c|c|c|c|c|c|c|c|c|c|c|c|c|c|c|c|}
\hline $\begin{array}{l}\text { MD04-2747 } \\
\text { CQ }\end{array}$ & 36.95 & 11.67 & 770 & Dinocyst & 2 & 18.56 & 0.00 & 0.00 & -0.19 & -0.59 & -0.86 & 1 & 18.56 & 0.00 & - & -0.19 & -0.59 & -0.86 \\
\hline $\begin{array}{l}\text { MD04- } \\
2797 C Q\end{array}$ & 36.95 & 11.67 & 771 & Foram & 2 & 17.73 & 1.28 & 0.90 & -1.01 & -1.42 & -1.69 & 1 & 18.37 & 0.00 & - & -0.38 & -0.77 & -1.05 \\
\hline $\begin{array}{l}\text { M40-4- } \\
\text { SL78/78MUC8 }\end{array}$ & 37.04 & 13.19 & 470 & Alkenone & 2 & 18.90 & 0.60 & 0.42 & -0.23 & 0.12 & -0.36 & 2 & 18.90 & 0.60 & 0.42 & -0.23 & 0.12 & -0.36 \\
\hline SU81-18 & 37.77 & -10.18 & 3135 & Alkenone & 2 & 18.25 & 0.70 & 0.49 & 0.59 & 0.33 & 0.44 & 0 & - & - & - & - & - & - \\
\hline MD95-2042 & 37.80 & -10.17 & 3146 & Alkenone & 3 & 18.60 & 0.20 & 0.10 & 0.95 & 0.68 & 0.79 & 1 & 18.50 & 0.00 & - & 0.85 & 0.58 & 0.69 \\
\hline MD01-2334 & 37.80 & -10.17 & 3166 & $\mathrm{Mg} / \mathrm{Ca}$ & 2 & - & - & - & - & - & - & 2 & - & - & - & - & - & - \\
\hline MD01-2443 & 37.88 & -10.18 & 2925 & Alkenone & 6 & 18.02 & 0.56 & 0.19 & 0.42 & 0.10 & 0.21 & 3 & 17.92 & 0.26 & 0.13 & 0.32 & 0.00 & 0.10 \\
\hline BS79-33 & 38.26 & 14.03 & 1282 & Alkenone & 1 & 20.12 & - & - & 1.12 & 0.73 & 0.92 & 1 & 20.12 & 0.00 & - & 1.12 & 0.73 & 0.92 \\
\hline BS79-38 & 38.41 & 13.58 & 1489 & Alkenone & 3 & 16.07 & 1.42 & 0.71 & -2.93 & -3.32 & -3.08 & 2 & 15.71 & 0.65 & 0.46 & -3.30 & -3.69 & -3.44 \\
\hline D13882 & 38.63 & -9.45 & 88 & Alkenone & 8 & 18.38 & 0.76 & 0.23 & - & 1.01 & 1.09 & 4 & 18.32 & 0.16 & 0.07 & - & 0.95 & 1.03 \\
\hline ODP 975 & 38.89 & 4.50 & 2426 & Foram & 2 & 18.38 & 0.46 & 0.32 & -0.60 & -0.92 & -0.61 & 1 & 18.60 & 0.00 & - & -0.37 & -0.69 & -0.38 \\
\hline ODP 974 & 40.35 & 12.13 & 3493 & Foram & 2 & 15.60 & 0.00 & 0.00 & -2.99 & -3.39 & -3.16 & 2 & 15.60 & 0.00 & 0.00 & -2.99 & -3.39 & -3.16 \\
\hline MD95-2040 & 40.58 & -9.86 & 2465 & Foram & 4 & 15.38 & 1.80 & 0.78 & -0.85 & -0.99 & -1.09 & 2 & 15.68 & 1.00 & 0.70 & -0.55 & -0.69 & -0.79 \\
\hline MD01-2430 & 40.80 & 27.73 & 580 & Dinocyst & 2 & 9.49 & 0.15 & 0.10 & - & -9.76 & -7.21 & 0 & - & - & - & - & - & - \\
\hline M44-KL71 & 40.84 & 27.76 & 566 & Alkenone & 5 & 18.32 & 1.10 & 0.54 & - & -0.93 & 1.62 & 3 & 18.27 & 1.10 & 0.57 & - & -0.99 & 1.57 \\
\hline AD91-17 & 40.87 & 18.64 & 844 & Alkenone & 3 & 16.63 & 0.10 & 0.06 & -1.02 & -2.62 & -2.07 & 1 & 16.60 & 0.00 & - & -1.06 & -2.65 & -2.10 \\
\hline $\mathrm{M} 40 / 480 \mathrm{SL}$ & 40.95 & 11.00 & 1881 & Foram & 2 & 15.73 & 0.05 & 0.04 & -2.34 & -2.98 & -2.80 & 1 & 15.71 & 0.00 & - & -2.36 & -3.00 & -2.83 \\
\hline ODP1019C & 41.68 & -124.93 & 980 & Alkenone & 9 & 10.54 & 1.00 & 0.35 & -1.89 & -2.00 & -2.16 & 3 & 10.77 & 0.10 & 0.06 & -1.66 & -1.77 & -1.93 \\
\hline CH69-K09 & 41.76 & -47.35 & 4100 & Foram & 3 & 12.57 & 1.80 & 0.99 & -2.99 & -3.67 & -3.53 & 2 & 12.81 & 1.80 & 1.28 & -2.76 & -3.43 & -3.29 \\
\hline IN68-9 & 41.78 & 17.90 & 1234 & Foram & 2 & 18.19 & 0.19 & 0.13 & 0.51 & -0.07 & -0.24 & 2 & 18.19 & 0.19 & 0.13 & 0.51 & -0.07 & -0.23 \\
\hline MD99-2346 & 42.07 & 4.15 & 2100 & Foram & 4 & 15.18 & 0.32 & 0.15 & -1.32 & -1.77 & -1.91 & 2 & 15.19 & 0.32 & 0.23 & -1.31 & -1.76 & -1.90 \\
\hline $\begin{array}{l}\text { M40/4 82-2- } \\
\text { SL }\end{array}$ & 42.31 & 3.77 & 1070 & Foram & 10 & 15.26 & 1.18 & 0.34 & -0.99 & -1.65 & -1.89 & 5 & 15.35 & 1.18 & 0.44 & -0.90 & -1.56 & -1.79 \\
\hline W8709A-8TC & 42.54 & -127.68 & 3111 & Alkenone & 1 & 11.40 & - & - & 0.94 & -1.82 & -1.82 & 0 & - & - & - & - & - & - \\
\hline $\begin{array}{l}\text { OCE326- } \\
\text { GGC26 }\end{array}$ & 43.48 & -54.87 & 3975 & Alkenone & 7 & 11.91 & 0.72 & 0.25 & 1.15 & 1.10 & 0.59 & 3 & 12.00 & 0.18 & 0.10 & 1.24 & 1.20 & 0.69 \\
\hline $\begin{array}{l}\text { OCE326- } \\
\text { GGC26 }\end{array}$ & 43.48 & -54.87 & 3975 & $\mathrm{Mg} / \mathrm{Ca}$ & 1 & - & - & - & - & - & - & 1 & - & - & - & - & - & - \\
\hline OCE326- & 43.88 & -62.80 & 250 & Alkenone & 15 & 13.01 & 2.09 & 0.64 & 5.30 & 4.89 & 3.79 & 8 & 12.92 & 2.01 & 0.84 & 5.21 & 4.80 & 3.70 \\
\hline
\end{tabular}




\begin{tabular}{|c|c|c|c|c|c|c|c|c|c|c|c|c|c|c|c|c|c|c|}
\hline GGC30 & & & & & & & & & & & & & & & & & & \\
\hline $\begin{array}{l}\mathrm{HU}-90-031- \\
044\end{array}$ & 44.49 & -55.19 & 1381 & Dinocyst & 1 & 8.10 & - & - & -0.28 & -0.46 & -0.78 & 1 & 8.10 & 0.00 & - & -0.28 & -0.46 & -0.78 \\
\hline MD01-2412 & 44.53 & 145.04 & 1225 & Alkenone & 14 & 11.94 & 1.53 & 0.50 & 6.03 & 5.06 & 5.36 & 7 & 11.79 & 1.33 & 0.49 & 5.88 & 4.91 & 5.21 \\
\hline MD-95-2033 & 44.66 & -55.62 & 1412 & Dinocyst & 4 & 9.20 & 0.85 & 0.40 & 1.13 & 0.64 & 0.32 & 2 & 8.87 & 0.07 & 0.05 & 0.80 & 0.31 & -0.01 \\
\hline $\begin{array}{l}\text { MR00-K03- } \\
\text { PC01 }\end{array}$ & 46.19 & 152.32 & 2793 & Alkenone & 4 & 7.75 & 1.00 & 0.64 & 3.41 & 3.35 & 3.15 & 1 & 7.30 & 0.00 & - & 2.96 & 2.90 & 2.70 \\
\hline $\begin{array}{l}\text { HU-2003-033- } \\
011\end{array}$ & 48.15 & -51.50 & 241 & Dinocyst & 5 & 0.05 & 1.43 & 0.69 & -3.53 & -3.62 & -4.57 & 3 & -0.02 & 1.43 & 0.74 & -3.60 & -3.68 & -4.64 \\
\hline GGC15 & 48.17 & 151.34 & 1980 & Alkenone & 1 & 9.50 & - & - & 5.73 & 5.61 & 5.18 & 0 & - & - & - & - & - & - \\
\hline MD-99-2220 & 48.60 & -68.63 & 320 & Dinocyst & 6 & 4.21 & 2.64 & 1.17 & - & -1.21 & - & 2 & 4.72 & 2.12 & 1.50 & - & -0.70 & - \\
\hline $\begin{array}{l}\text { BENGAL } \\
13078 \# 16\end{array}$ & 48.83 & -16.45 & 4844 & Foram & 2 & 16.88 & 2.48 & 1.75 & 3.17 & 3.01 & 2.94 & 1 & 18.12 & 0.00 & - & 4.41 & 4.25 & 4.17 \\
\hline JT96-09 & 48.90 & -126.88 & 920 & Alkenone & 2 & 10.15 & 0.70 & 0.49 & -0.72 & -1.10 & -1.09 & 0 & - & - & - & - & - & - \\
\hline $\begin{array}{l}\text { HU-90-031- } \\
019\end{array}$ & 49.29 & -63.99 & 322 & Dinocyst & 4 & 3.39 & 0.49 & 0.21 & -1.54 & -1.40 & -1.99 & 2 & 3.35 & 0.49 & 0.35 & -1.58 & -1.44 & -2.04 \\
\hline PC4 & 49.49 & 146.13 & 664 & Alkenone & 3 & 7.91 & 1.19 & 0.62 & 4.70 & 3.79 & - & 1 & 7.71 & 0.00 & - & 4.50 & 3.59 & - \\
\hline $\begin{array}{l}\text { HU-91-045- } \\
094\end{array}$ & 50.20 & -45.69 & 3448 & Dinocyst & 2 & 6.23 & 1.52 & 1.08 & -1.70 & -1.71 & -1.45 & 1 & 7.00 & 0.00 & - & -0.94 & -0.94 & -0.69 \\
\hline PC2 & 50.40 & 148.32 & 1258 & Alkenone & 2 & 7.71 & 0.85 & 0.60 & 3.99 & 3.53 & 3.11 & 2 & 7.71 & 0.85 & 0.60 & 4.00 & 3.53 & 3.11 \\
\hline MD01-2416 & 51.00 & 168.00 & 2317 & $\mathrm{Mg} / \mathrm{Ca}$ & 12 & - & - & - & - & - & - & 4 & - & - & - & - & - & - \\
\hline $\mathrm{CH} 77-02$ & 52.07 & -36.08 & 3744 & Foram & 8 & 7.97 & 1.77 & 0.60 & -1.34 & -0.89 & -1.20 & 5 & 7.70 & 0.34 & 0.14 & -1.61 & -1.16 & -1.47 \\
\hline $\begin{array}{l}\text { HU-91-045- } \\
080\end{array}$ & 53.06 & -33.53 & 3024 & Dinocyst & 1 & 12.13 & - & - & 2.95 & 3.25 & 3.10 & 1 & 12.13 & 0.00 & - & 2.95 & 3.25 & 3.10 \\
\hline $\begin{array}{l}\text { HU-91-045- } \\
085\end{array}$ & 53.98 & -38.64 & 3603 & Dinocyst & 5 & 10.49 & 3.29 & 1.49 & 2.93 & 2.52 & 2.38 & 3 & 9.52 & 1.88 & 0.97 & 1.96 & 1.56 & 1.42 \\
\hline NEAP17K & 54.68 & -28.35 & 2734 & Foram & 9 & 10.55 & 2.14 & 0.72 & 0.64 & 0.63 & 0.73 & 4 & 10.81 & 1.96 & 0.83 & 0.90 & 0.89 & 0.99 \\
\hline NA87-22 & 55.50 & -14.07 & 2161 & Foram & 6 & 11.65 & 1.44 & 0.47 & 0.20 & 0.17 & 0.17 & 2 & 11.71 & 0.17 & 0.12 & 0.25 & 0.23 & 0.22 \\
\hline MD-99-2254 & 56.80 & -30.66 & 2440 & Dinocyst & 7 & 7.01 & 6.16 & 2.05 & -1.62 & -1.78 & -1.84 & 4 & 5.29 & 1.00 & 0.61 & -3.34 & -3.50 & -3.56 \\
\hline MD99-2251 & 57.43 & -27.90 & 2620 & $\mathrm{Mg} / \mathrm{Ca}$ & 17 & - & - & - & - & - & - & 7 & - & - & - & - & - & - \\
\hline IOW225517 & 57.67 & 7.09 & 293 & Alkenone & 11 & 13.76 & 1.00 & 0.35 & 4.69 & 4.20 & 3.87 & 5 & 13.76 & 1.00 & 0.36 & 4.68 & 4.20 & 3.87 \\
\hline IOW225514 & 57.84 & 8.70 & 420 & Alkenone & 16 & 13.28 & 1.00 & 0.26 & 4.28 & 3.75 & 3.46 & 8 & 13.21 & 0.90 & 0.30 & 4.22 & 3.69 & 3.39 \\
\hline
\end{tabular}




\begin{tabular}{|c|c|c|c|c|c|c|c|c|c|c|c|c|c|c|c|c|c|c|}
\hline MD-99-2227 & 58.21 & -48.37 & 3460 & Dinocyst & 4 & 5.06 & 4.13 & 1.81 & 0.35 & 0.69 & 0.43 & 2 & 5.70 & 2.80 & 1.98 & 0.98 & 1.33 & 1.07 \\
\hline $\begin{array}{l}\text { HU-90-013- } \\
013\end{array}$ & 58.21 & -48.31 & 3380 & Dinocyst & 5 & 4.86 & 1.50 & 0.59 & 0.14 & 0.49 & 0.23 & 3 & 5.14 & 1.07 & 0.54 & 0.42 & 0.77 & 0.51 \\
\hline $\begin{array}{l}\text { HU-84-030- } \\
021 \text { TWC }\end{array}$ & 58.37 & -57.51 & 2853 & Dinocyst & 7 & 4.78 & 2.28 & 1.09 & 1.36 & 1.46 & 1.89 & 3 & 4.44 & 2.40 & 1.20 & 1.02 & 1.12 & 1.55 \\
\hline $\begin{array}{l}\text { HU-84-030- } \\
021 \text { PC }\end{array}$ & 58.37 & -57.51 & 2853 & Dinocyst & 8 & 3.91 & 4.36 & 1.34 & 0.49 & 0.59 & 1.02 & 3 & 4.73 & 3.41 & 1.71 & 1.31 & 1.41 & 1.84 \\
\hline MD95-2015 & 58.76 & -25.96 & 2630 & Alkenone & 11 & 11.63 & 1.47 & 0.43 & 2.38 & 2.06 & 2.10 & 6 & 11.62 & 0.59 & 0.27 & 2.37 & 2.05 & 2.09 \\
\hline MD95-2015 & 58.76 & -25.96 & 2630 & Foram & 3 & 9.50 & 0.33 & 0.16 & 0.25 & -0.07 & -0.03 & 1 & 9.33 & 0.00 & - & 0.08 & -0.24 & -0.21 \\
\hline $\begin{array}{l}\mathrm{HU}-91-045- \\
072\end{array}$ & 58.94 & -28.74 & 2237 & Dinocyst & 11 & 6.10 & 1.84 & 0.55 & -2.50 & -2.78 & -2.81 & 5 & 6.07 & 0.48 & 0.24 & -2.53 & -2.81 & -2.84 \\
\hline HM03-133-25 & 60.11 & -6.07 & 1156 & Dinocyst & 9 & 6.87 & 5.00 & 1.95 & -2.87 & -2.70 & -2.71 & 4 & 6.81 & 4.49 & 2.32 & -2.93 & -2.76 & -2.77 \\
\hline ODP984 & 61.00 & -25.00 & 1648 & $\mathrm{Mg} / \mathrm{Ca}$ & 11 & - & - & - & - & - & - & 6 & - & - & - & - & - & - \\
\hline LINK14 & 61.80 & -6.30 & 346 & Foram & 3 & 7.72 & 0.55 & 0.30 & -0.77 & -1.25 & -1.21 & 1 & 7.38 & 0.00 & - & -1.12 & -1.58 & -1.55 \\
\hline RAPID-12-1K & 62.09 & -17.82 & 1938 & $\mathrm{Mg} / \mathrm{Ca}$ & 5 & - & - & - & - & - & - & 3 & - & - & - & - & - & - \\
\hline MD99-2284 & 62.37 & -0.98 & 1500 & Foram & 8 & 7.54 & 2.16 & 0.71 & -1.10 & -1.28 & -1.42 & 4 & 7.23 & 1.98 & 0.90 & -1.40 & -1.59 & -1.73 \\
\hline MD-99-2269 & 66.64 & -20.86 & 365 & Dinocyst & 20 & 3.61 & 2.21 & 0.49 & -0.79 & -1.29 & -0.33 & 10 & 3.64 & 0.96 & 0.35 & -0.76 & -1.26 & -0.29 \\
\hline MD95-2011 & 66.97 & 7.63 & 1048 & Alkenone & 8 & 12.43 & 1.10 & 0.42 & 4.20 & 3.91 & 4.06 & 4 & 12.64 & 0.62 & 0.28 & 4.41 & 4.12 & 4.27 \\
\hline MD95-2011 & 66.97 & 7.63 & 1048 & Foram & 10 & 7.68 & 0.79 & 0.27 & -0.55 & -0.84 & -0.69 & 6 & 7.74 & 0.79 & 0.31 & -0.49 & -0.78 & -0.63 \\
\hline JR51-GC35 & 67.00 & -17.96 & 420 & Alkenone & 11 & 6.92 & 3.97 & 1.18 & 3.95 & 4.06 & 2.96 & 5 & 6.97 & 3.98 & 1.58 & 4.00 & 4.11 & 3.02 \\
\hline M23323 & 67.77 & 5.92 & 1286 & Dinocyst & 10 & 5.72 & 2.53 & 0.70 & -2.19 & -2.39 & -2.06 & 5 & 5.95 & 1.20 & 0.50 & -1.96 & -2.16 & -1.83 \\
\hline JM-96-1207 & 68.10 & -29.35 & 404 & Dinocyst & 9 & 3.11 & 1.49 & 0.56 & 2.11 & 3.52 & - & 5 & 3.35 & 1.05 & 0.44 & 2.35 & 3.77 & - \\
\hline 2005-804-006 & 68.99 & -106.57 & 118 & Dinocyst & 6 & -0.44 & 0.68 & 0.24 & - & -0.10 & - & 3 & -0.33 & 0.23 & 0.12 & - & 0.01 & - \\
\hline PL-96-112 & 71.27 & 42.61 & 286 & Dinocyst & 9 & 3.55 & 3.11 & 1.32 & 0.54 & 0.44 & 0.42 & 5 & 3.59 & 3.21 & 1.39 & 0.58 & 0.49 & 0.46 \\
\hline HLY0501-05 & 72.69 & -157.52 & 415 & Dinocyst & 6 & 0.52 & 2.53 & 0.89 & 1.58 & 1.43 & - & 2 & 0.49 & 0.08 & 0.06 & 1.56 & 1.40 & - \\
\hline 2004-804-009 & 74.19 & -81.20 & 781 & Dinocyst & 6 & 0.28 & 1.95 & 0.72 & - & 0.47 & - & 3 & 0.11 & 1.19 & 0.67 & - & 0.29 & - \\
\hline 2005-804-004 & 74.27 & -91.09 & 350 & Dinocyst & 13 & -1.00 & 1.29 & 0.35 & - & 0.13 & - & 7 & -1.07 & 0.47 & 0.17 & - & 0.06 & - \\
\hline M23258-2 & 75.00 & 13.97 & 1768 & Alkenone & 4 & 7.03 & 0.52 & 0.23 & 3.00 & 3.09 & 2.45 & 2 & 7.16 & 0.11 & 2.10 & 3.13 & 3.21 & 2.57 \\
\hline $23258-2$ & 75.00 & 13.97 & 1768 & Foram & 17 & 2.68 & 3.21 & 0.84 & -1.35 & -1.26 & -1.90 & 9 & 2.66 & 2.87 & 0.85 & -1.37 & -1.29 & -1.93 \\
\hline $\begin{array}{l}\text { HU-91-039- } \\
008\end{array}$ & 77.27 & -74.32 & 663 & Dinocyst & 4 & 0.76 & 1.22 & 0.59 & 1.35 & 1.59 & - & 4 & 0.76 & 1.22 & 0.59 & 1.35 & 1.59 & - \\
\hline
\end{tabular}


Table 3: Summery information for individual summer records

\begin{tabular}{|c|c|c|c|c|c|c|c|c|c|c|c|c|c|c|c|c|c|c|}
\hline \multicolumn{5}{|c|}{ SUMMER } & \multicolumn{7}{|c|}{ 5.5-6.5 ka BP } & \multicolumn{7}{|c|}{ 5.75-6.25 ka BP } \\
\hline $\begin{array}{l}\frac{0}{0} \\
\text { ப் }\end{array}$ & 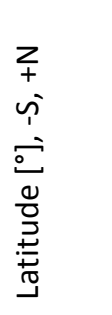 & 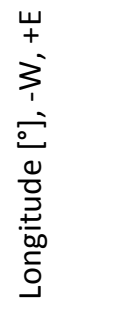 & 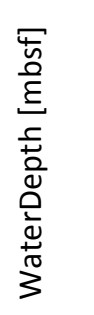 & 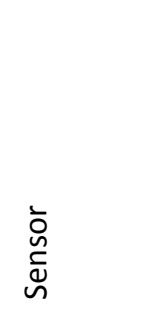 & 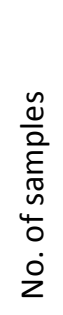 & 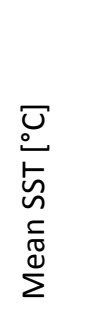 & 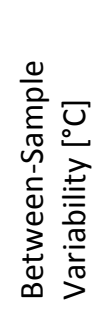 & 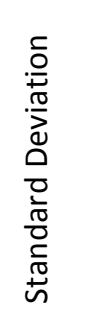 & 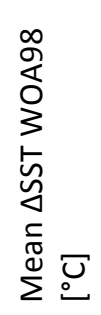 & 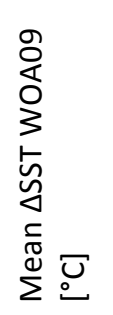 & 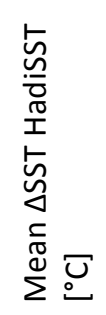 & 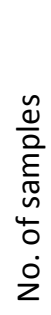 & 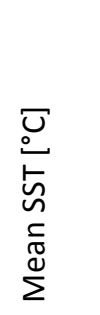 & 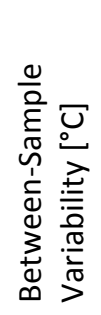 & 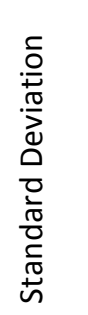 & 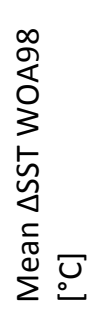 & 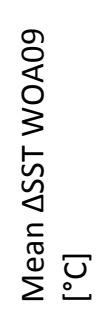 & 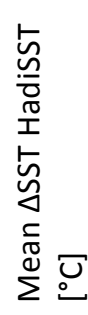 \\
\hline MD94-103 & -45.58 & 86.53 & 3560 & Alkenone & 4 & - & - & - & - & - & - & 3 & - & - & - & - & - & - \\
\hline MD97-2120 & -45.53 & 174.93 & 1210 & Alkenone & 7 & - & - & - & - & - & - & 3 & - & - & - & - & - & - \\
\hline MD97-2120 & -45.53 & 174.93 & 1210 & $\mathrm{Mg} / \mathrm{Ca}$ & 1 & 12.23 & - & - & -1.26 & -1.65 & -1.55 & 1 & 12.23 & 0.00 & - & -1.26 & -1.65 & -1.55 \\
\hline S0136-GC11 & -43.44 & 167.85 & 1556 & Alkenone & 3 & - & - & - & - & - & - & 1 & - & - & - & - & - & - \\
\hline TN057-21PC & -41.13 & 8.80 & 4981 & Alkenone & 6 & - & - & - & - & - & - & 3 & - & - & - & - & - & - \\
\hline ODP1233 & -41.01 & -74.45 & 838 & Alkenone & 3 & - & - & - & - & - & - & 2 & - & - & - & - & - & - \\
\hline GeoB3313-1 & -41.00 & -74.45 & 852 & Alkenone & 10 & - & - & - & - & - & - & 4 & - & - & - & - & - & - \\
\hline MD97-2121 & -40.38 & 177.99 & 3014 & Alkenone & 15 & - & - & - & - & - & - & 8 & - & - & - & - & - & - \\
\hline MD03-2611 & -36.73 & 136.55 & 2420 & Alkenone & 3 & - & - & - & - & - & - & 1 & - & - & - & - & - & - \\
\hline GIK17748-2 & -32.75 & -72.03 & 2545 & Alkenone & 2 & - & - & - & - & - & - & 1 & - & - & - & - & - & - \\
\hline $\begin{array}{l}\text { GIK17748-2 } \\
\text { (SO80-4) }\end{array}$ & -32.75 & -72.03 & 2545 & Foram & 2 & 14.82 & 1.29 & 0.91 & -2.44 & -2.68 & -2.22 & 1 & 14.17 & 0.00 & - & -3.08 & -3.33 & -2.87 \\
\hline GeoB7139-2 & -30.20 & -71.98 & 3270 & Alkenone & 2 & - & - & - & - & - & - & 1 & - & - & - & - & - & - \\
\hline GeoB7139-2 & -30.20 & -71.98 & 3269 & Foram & 2 & 14.18 & 0.06 & 0.04 & -3.97 & -3.97 & -3.46 & 1 & 14.21 & 0.00 & - & -3.95 & -3.94 & -3.43 \\
\hline ODP1084B & -25.51 & 13.28 & 1992 & $\mathrm{Mg} / \mathrm{Ca}$ & 8 & 13.69 & 1.70 & 0.74 & -3.29 & -3.60 & -5.47 & 3 & 13.43 & 0.80 & 0.42 & -3.55 & -3.86 & -5.73 \\
\hline GeoB7112-5 & -24.03 & -70.82 & 2507 & Foram & 3 & 14.01 & 0.63 & 0.35 & -7.33 & -7.66 & -6.65 & 2 & 13.90 & 0.60 & 0.42 & -7.44 & -7.77 & -6.76 \\
\hline GeoB1710-3 & -23.43 & 11.69 & 1045 & Alkenone & 1 & - & - & - & - & - & - & 1 & - & - & - & - & - & - \\
\hline GeoB1711-4 & -23.31 & 12.37 & 1967 & Alkenone & 1 & - & - & - & - & - & - & 1 & - & - & - & - & - & - \\
\hline GeoB1712-4 & -23.26 & 12.81 & 998 & Alkenone & 2 & - & - & - & - & - & - & 2 & - & - & - & - & - & - \\
\hline MD97-2125 & -22.57 & 161.73 & 1684 & $\mathrm{Mg} / \mathrm{Ca}$ & 2 & 26.64 & 0.57 & 0.40 & 0.31 & 0.08 & 0.19 & 0 & - & - & - & - & - & - \\
\hline MD79257 & -20.40 & 36.33 & 1262 & Alkenone & 2 & - & - & - & - & - & - & 1 & - & - & - & - & - & - \\
\hline
\end{tabular}




\begin{tabular}{|c|c|c|c|c|c|c|c|c|c|c|c|c|c|c|c|c|c|c|}
\hline GeoB1023-5 & -17.16 & 11.01 & 1978 & Alkenone & 3 & - & - & - & - & - & - & 1 & - & - & - & - & - & - \\
\hline MD01-2378 & -13.08 & 121.79 & 1783 & $\mathrm{Mg} / \mathrm{Ca}$ & 8 & 28.09 & 1.21 & 0.40 & -1.40 & -1.18 & -1.27 & 4 & 28.08 & 0.83 & 0.34 & -1.41 & -1.19 & -1.28 \\
\hline ODP1078C & -11.92 & 13.40 & 426 & Alkenone & 3 & - & - & - & - & - & - & 1 & - & - & - & - & - & - \\
\hline MD05-2928 & -11.29 & 148.86 & 2250 & Alkenone & 3 & - & - & - & - & - & - & 1 & - & - & - & - & - & - \\
\hline MD98-2170 & -10.59 & 125.39 & 832 & $\mathrm{Mg} / \mathrm{Ca}$ & 4 & 29.57 & 0.56 & 0.23 & -0.15 & -0.02 & 0.48 & 1 & 29.54 & 0.00 & - & -0.18 & -0.05 & 0.45 \\
\hline MD98-2165 & -9.65 & 118.34 & 2100 & $\mathrm{Mg} / \mathrm{Ca}$ & 5 & 26.72 & 1.17 & 0.44 & -2.34 & -2.46 & -2.07 & 2 & 26.68 & 0.33 & 0.23 & -2.39 & -2.51 & -2.11 \\
\hline SO139-74KL & -6.54 & 103.83 & 1690 & Alkenone & 24 & - & - & - & - & - & - & 12 & - & - & - & - & - & - \\
\hline GeoB10038-4 & -5.94 & 103.25 & 1819 & Alkenone & 2 & - & - & - & - & - & - & 1 & - & - & - & - & - & - \\
\hline GeoB10038-4 & -5.93 & 103.25 & 1819 & $\mathrm{Mg} / \mathrm{Ca}$ & 1 & 26.02 & - & - & -2.30 & -2.40 & -2.41 & 1 & 26.02 & 0.00 & - & -2.30 & -2.40 & -2.41 \\
\hline GeoB6518-1 & -5.59 & 11.22 & 962 & Alkenone & 6 & - & - & - & - & - & - & 2 & - & - & - & - & - & - \\
\hline RC24-16 & -5.04 & -10.19 & 3543 & Foram & 2 & 27.19 & 0.51 & 0.36 & 0.41 & 0.31 & 0.23 & 0 & - & - & - & - & - & - \\
\hline MD98-2176 & -5.00 & 133.45 & 2382 & $\mathrm{Mg} / \mathrm{Ca}$ & 24 & 29.33 & 1.50 & 0.43 & 0.15 & 0.25 & 0.67 & 11 & 29.53 & 1.20 & 0.37 & 0.35 & 0.44 & 0.87 \\
\hline MD98-2162 & -4.69 & 117.90 & 1855 & $\mathrm{Mg} / \mathrm{Ca}$ & 2 & 29.42 & 0.31 & 0.22 & 0.58 & 0.51 & 0.86 & 1 & 29.58 & 0.00 & - & 0.74 & 0.67 & 1.02 \\
\hline GeoB 3129-1 & -4.61 & -36.64 & 830 & Alkenone & 3 & - & - & - & - & - & - & 1 & - & - & - & - & - & - \\
\hline $\begin{array}{l}\text { GeoB3129/ } \\
3911\end{array}$ & -4.61 & -36.64 & 830 & $\mathrm{Mg} / \mathrm{Ca}$ & 3 & 27.18 & 0.61 & 0.32 & -0.73 & -0.49 & -0.61 & 1 & 27.05 & 0.00 & - & -0.86 & -0.62 & -0.74 \\
\hline GeoB3910-2 & -4.02 & -36.35 & 2362 & Alkenone & 2 & - & - & - & - & - & - & 1 & - & - & - & - & - & - \\
\hline V19-30 & -3.38 & -83.52 & 3091 & Alkenone & 1 & - & - & - & - & - & - & 1 & - & - & - & - & - & - \\
\hline V19-28 & -2.51 & -84.65 & 2720 & Alkenone & 1 & - & - & - & - & - & - & 1 & - & - & - & - & - & - \\
\hline V19-28 & -2.37 & -84.65 & 2720 & $\mathrm{Mg} / \mathrm{Ca}$ & 1 & 20.94 & - & - & -3.73 & -4.16 & -4.93 & 1 & 20.94 & 0.00 & - & -3.73 & -4.16 & -4.93 \\
\hline GeoB10029-4 & -1.50 & 100.13 & 772 & $\mathrm{Mg} / \mathrm{Ca}$ & 2 & 28.48 & 0.15 & 0.11 & - & -0.55 & -0.19 & 2 & 28.48 & 0.15 & 0.11 & - & -0.55 & -0.19 \\
\hline V21-30 & -1.22 & -89.68 & 617 & Alkenone & 3 & - & - & - & - & - & - & 1 & - & - & - & - & - & - \\
\hline V21-30 & -1.22 & -89.68 & 617 & $\mathrm{Mg} / \mathrm{Ca}$ & 5 & 21.43 & 0.43 & 0.18 & -3.39 & -4.24 & -4.54 & 1 & 21.55 & 0.00 & - & -3.27 & -4.12 & -4.42 \\
\hline GIK16773-1 & -0.97 & -9.44 & 4662 & Foram & 2 & 27.14 & 0.07 & 0.05 & -0.27 & -0.52 & -0.30 & 1 & 27.18 & 0.00 & - & -0.22 & -0.48 & -0.26 \\
\hline V19-27 & -0.47 & -82.67 & 1373 & Alkenone & 1 & - & - & - & - & - & - & 1 & - & - & - & - & - & - \\
\hline ODP1240 & 0.02 & -86.45 & 2921 & $\mathrm{Mg} / \mathrm{Ca}$ & 8 & 25.59 & 0.55 & 0.20 & 1.97 & 1.47 & 1.59 & 4 & 25.61 & 0.54 & 0.27 & 1.98 & 1.49 & 1.61 \\
\hline TR163-22 & 0.52 & -92.40 & 2830 & $\mathrm{Mg} / \mathrm{Ca}$ & 3 & 24.07 & 0.40 & 0.21 & 1.56 & 1.27 & 1.05 & 2 & 24.15 & 0.30 & 0.21 & 1.64 & 1.35 & 1.13 \\
\hline $\begin{array}{l}\text { ME0005A- } \\
24 J C\end{array}$ & 1.50 & -86.49 & 2941 & Alkenone & 3 & - & - & - & - & - & - & 2 & - & - & - & - & - & - \\
\hline TR163-19 & 2.26 & -90.95 & 2348 & $\mathrm{Mg} / \mathrm{Ca}$ & 1 & 26.20 & - & - & 0.83 & 0.64 & 0.66 & 0 & - & - & - & - & - & - \\
\hline GeoB4905-4 & 2.50 & 9.39 & 1328 & Alkenone & 6 & - & - & - & - & - & - & 3 & - & - & - & - & - & - \\
\hline GeoB4905-4 & 2.50 & 9.39 & 1328 & $\mathrm{Mg} / \mathrm{Ca}$ & 9 & 26.41 & 1.62 & 0.59 & 0.29 & -0.19 & 0.56 & 6 & 26.50 & 1.62 & 0.67 & 0.38 & -0.10 & 0.65 \\
\hline
\end{tabular}




\begin{tabular}{|c|c|c|c|c|c|c|c|c|c|c|c|c|c|c|c|c|c|c|}
\hline MD03-2707 & 2.50 & 9.40 & 1295 & $\mathrm{Mg} / \mathrm{Ca}$ & 24 & 26.87 & 1.99 & 0.42 & 0.75 & 0.27 & 1.02 & 12 & 26.91 & 1.42 & 0.48 & 0.79 & 0.31 & 1.07 \\
\hline MD85674 & 3.18 & 50.43 & 4875 & Alkenone & 2 & - & - & - & - & - & - & 0 & - & - & - & - & - & - \\
\hline $\begin{array}{l}\text { KNR176- } \\
\text { JPC32 }\end{array}$ & 4.85 & -77.96 & 2200 & Alkenone & 4 & - & - & - & - & - & - & 2 & - & - & - & - & - & - \\
\hline V30-36 & 5.35 & -27.32 & 4245 & Foram & 1 & 27.25 & - & - & -0.32 & -0.27 & 0.02 & 1 & 27.25 & 0.00 & - & -0.32 & -0.27 & 0.02 \\
\hline GIK18287-3 & 5.60 & 110.60 & 598 & Foram & 3 & 28.55 & 0.28 & 0.15 & -0.30 & -0.30 & -0.15 & 1 & 28.66 & 0.00 & - & -0.19 & -0.19 & -0.04 \\
\hline $18287-3$ & 5.65 & 110.65 & 598 & Alkenone & 3 & - & - & - & - & - & - & 1 & - & - & - & - & - & - \\
\hline GIK18287-3 & 5.98 & 110.65 & 598 & Alkenone & 3 & - & - & - & - & - & - & 1 & - & - & - & - & - & - \\
\hline 17964 & 6.16 & 112.21 & 1556 & Alkenone & 2 & - & - & - & - & - & - & 0 & - & - & - & - & - & - \\
\hline MD98-2181 & 6.30 & 125.83 & 2114 & $\mathrm{Mg} / \mathrm{Ca}$ & 22 & 29.55 & 1.38 & 0.35 & 0.58 & 0.51 & 1.01 & 12 & 29.51 & 1.13 & 0.40 & 0.54 & 0.46 & 0.96 \\
\hline MD01-2390 & 6.63 & 113.40 & 1545 & Foram & 2 & 28.92 & 0.08 & 0.06 & 0.10 & 0.10 & 0.19 & 1 & 28.88 & 0.00 & - & 0.05 & 0.06 & 0.15 \\
\hline MD01-2390 & 6.64 & 113.41 & 1545 & $\mathrm{Mg} / \mathrm{Ca}$ & 3 & 28.28 & 1.15 & 0.65 & -0.55 & -0.54 & -0.45 & 1 & 29.03 & 0.00 & - & 0.20 & 0.21 & 0.30 \\
\hline $\begin{array}{l}\text { ODP1242/ME } \\
0005 \mathrm{~A}-43 \mathrm{JC}\end{array}$ & 7.86 & -83.61 & 1366 & $\mathrm{Mg} / \mathrm{Ca}$ & 9 & 26.47 & 1.99 & 0.58 & -1.37 & -1.46 & -1.27 & 4 & 26.53 & 0.36 & 0.17 & -1.32 & -1.40 & -1.22 \\
\hline MD02-2529 & 8.21 & -84.12 & 1619 & Alkenone & 3 & - & - & - & - & - & - & 2 & - & - & - & - & - & - \\
\hline MD97-2151 & 8.73 & 109.87 & 1598 & Alkenone & 10 & - & - & - & - & - & - & 5 & - & - & - & - & - & - \\
\hline MD972151 & 8.73 & 109.87 & 283 & Foram & 3 & 28.69 & 0.70 & 0.39 & 0.05 & 0.06 & 0.16 & 1 & 28.90 & 0.00 & - & 0.26 & 0.27 & 0.37 \\
\hline MD97-2141 & 8.80 & 121.30 & 3633 & $\mathrm{Mg} / \mathrm{Ca}$ & 15 & 29.04 & 1.79 & 0.53 & 0.30 & 0.32 & 0.35 & 7 & 29.19 & 1.40 & 0.61 & 0.45 & 0.47 & 0.50 \\
\hline $18252-3$ & 9.23 & 109.38 & 1273 & Alkenone & 3 & - & - & - & - & - & - & 1 & - & - & - & - & - & - \\
\hline MD01-2392 & 9.85 & 110.21 & 1966 & Alkenone & 1 & - & - & - & - & - & - & 1 & - & - & - & - & - & - \\
\hline MD77194 & 10.47 & 75.23 & 1222 & Alkenone & 2 & - & - & - & - & - & - & 1 & - & - & - & - & - & - \\
\hline PL07-39PC & 10.70 & -65.94 & 790 & $\mathrm{Mg} / \mathrm{Ca}$ & 4 & 26.40 & 1.00 & 0.42 & -0.92 & -1.93 & -1.12 & 2 & 26.10 & 0.40 & 0.28 & -1.22 & -2.23 & -1.42 \\
\hline ODP $1002 C$ & 10.71 & -65.17 & 893 & Alkenone & 1 & - & - & - & - & - & - & 1 & - & - & - & - & - & - \\
\hline TY93-905 & 11.07 & 51.95 & 1567 & Alkenone & 7 & - & - & - & - & - & - & 4 & - & - & - & - & - & - \\
\hline VM28-122 & 11.57 & -78.42 & 3623 & $\mathrm{Mg} / \mathrm{Ca}$ & 1 & 27.60 & - & - & -0.49 & -0.49 & -0.68 & 1 & 27.60 & 0.00 & - & -0.49 & -0.49 & -0.68 \\
\hline M35003-4 & 12.08 & -61.25 & 1299 & Alkenone & 4 & - & - & - & - & - & - & 2 & - & - & - & - & - & - \\
\hline M35003-4 & 12.09 & -61.24 & 1299 & Foram & 3 & 28.32 & 0.15 & 0.07 & 0.15 & 0.11 & 0.05 & 2 & 28.32 & 0.14 & 0.10 & 0.14 & 0.11 & 0.05 \\
\hline MD972142 & 12.69 & 119.47 & 1557 & Foram & 4 & 28.75 & 0.17 & 0.08 & -0.21 & -0.12 & 0.09 & 3 & 28.74 & 0.18 & 0.09 & -0.22 & -0.13 & 0.07 \\
\hline ODP999 & 12.75 & -78.73 & 2827 & $\mathrm{Mg} / \mathrm{Ca}$ & 1 & 28.30 & - & - & 0.23 & 0.29 & 0.04 & 1 & 28.30 & 0.00 & - & 0.23 & 0.29 & 0.04 \\
\hline MD012394 & 13.78 & 110.25 & 2097 & Foram & 5 & 28.25 & 0.56 & 0.20 & 0.27 & -0.03 & -0.24 & 2 & 28.26 & 0.04 & 0.03 & 0.28 & -0.02 & -0.23 \\
\hline $74 \mathrm{KL}$ & 14.32 & 57.35 & 3212 & Alkenone & 2 & - & - & - & - & - & - & 1 & - & - & - & - & - & - \\
\hline SCS90-36 & 18.00 & 111.49 & 2050 & Alkenone & 2 & - & - & - & - & - & - & 1 & - & - & - & - & - & - \\
\hline
\end{tabular}




\begin{tabular}{|c|c|c|c|c|c|c|c|c|c|c|c|c|c|c|c|c|c|c|}
\hline BOFS $31 \mathrm{~K}$ & 19.00 & -20.17 & 3300 & Alkenone & 1 & - & - & - & - & - & - & 0 & - & - & - & - & - & - \\
\hline BOFS31K & 19.00 & -20.17 & 3300 & $\mathrm{Mg} / \mathrm{Ca}$ & 1 & 17.90 & - & - & -6.64 & -6.40 & -6.72 & 0 & - & - & - & - & - & - \\
\hline MD05-2904 & 19.45 & 116.25 & 2066 & $\mathrm{Mg} / \mathrm{Ca}$ & 4 & 27.16 & 0.54 & 0.24 & -1.56 & -1.59 & -1.60 & 2 & 27.05 & 0.49 & 0.35 & -1.68 & -1.71 & -1.72 \\
\hline ODP1145 & 19.58 & 117.63 & 3175 & $\mathrm{Mg} / \mathrm{Ca}$ & 1 & 27.80 & - & - & -0.95 & -1.00 & -0.98 & 1 & 27.80 & 0.00 & - & -0.95 & -1.00 & -0.98 \\
\hline $17938-2$ & 19.78 & 117.53 & 284 & Foram & 1 & 28.36 & - & - & -0.36 & -0.44 & -0.42 & 0 & - & - & - & - & - & - \\
\hline MD972148 & 19.80 & 117.54 & 4872 & Foram & 3 & 27.38 & 0.86 & 0.48 & -1.34 & -1.42 & -1.40 & 1 & 26.83 & 0.00 & - & -1.89 & -1.97 & -1.95 \\
\hline S093-126KL & 19.97 & 90.03 & 1253 & Alkenone & 2 & - & - & - & - & - & - & 0 & - & - & - & - & - & - \\
\hline $17940-2$ & 20.12 & 117.38 & 1727 & Alkenone & 8 & - & - & - & - & - & - & 8 & - & - & - & - & - & - \\
\hline MD97-2146 & 20.12 & 117.38 & 172 & Foram & 21 & 28.46 & 0.88 & 0.22 & -0.21 & -0.25 & -0.26 & 10 & 28.47 & 0.88 & 0.26 & -0.21 & -0.24 & -0.25 \\
\hline GeoB7926-2 & 20.22 & -18.45 & 2500 & Alkenone & 2 & - & - & - & - & - & - & 1 & - & - & - & - & - & - \\
\hline ODP658C & 20.75 & -18.58 & 2262 & Alkenone & 8 & - & - & - & - & - & - & 4 & - & - & - & - & - & - \\
\hline PC17 & 21.36 & -158.19 & 503 & Alkenone & 3 & - & - & - & - & - & - & 1 & - & - & - & - & - & - \\
\hline LAPAZ21P & 22.99 & -109.47 & 624 & Alkenone & 1 & - & - & - & - & - & - & 1 & - & - & - & - & - & - \\
\hline SO90-93KL & 23.58 & 64.22 & 1802 & Alkenone & 3 & - & - & - & - & - & - & 2 & - & - & - & - & - & - \\
\hline RN96-PC1 & 24.97 & 122.93 & 1676 & Foram & 4 & 28.14 & 0.33 & 0.15 & -0.23 & -0.48 & -0.03 & 1 & 28.24 & 0.00 & - & -0.13 & -0.38 & 0.07 \\
\hline 255 & 25.20 & 123.12 & 1575 & Foram & 5 & 28.67 & 0.45 & 0.20 & 0.31 & 0.23 & 0.48 & 3 & 28.79 & 0.34 & 0.17 & 0.43 & 0.34 & 0.59 \\
\hline RN88-PC5 & 25.26 & 125.15 & 2051 & Foram & 2 & 27.85 & 0.38 & 0.27 & -0.67 & -0.74 & -0.64 & 2 & 27.85 & 0.38 & 0.27 & -0.68 & -0.74 & -0.64 \\
\hline RN93-PC6 & 25.68 & 124.36 & 1849 & Foram & 2 & 28.04 & 0.16 & 0.11 & -0.36 & -0.48 & -0.34 & 0 & - & - & - & - & - & - \\
\hline RN93-PC4 & 26.55 & 125.07 & 1440 & Foram & 2 & 27.97 & 0.02 & 0.01 & -0.35 & -0.48 & -0.39 & 1 & 27.96 & 0.00 & - & -0.36 & -0.49 & -0.40 \\
\hline E017 & 26.57 & 126.03 & 1826 & Foram & 3 & 28.58 & 0.33 & 0.17 & 0.19 & 0.07 & 0.14 & 1 & 28.63 & 0.00 & - & 0.23 & 0.12 & 0.19 \\
\hline MD012404 & 26.65 & 125.81 & 1397 & Foram & 9 & 28.22 & 0.96 & 0.30 & -0.15 & -0.23 & -0.14 & 4 & 28.18 & 0.96 & 0.40 & -0.18 & -0.27 & -0.18 \\
\hline MD98-2193 & 27.40 & 126.27 & 1614 & Foram & 1 & 28.10 & - & - & -0.15 & -0.28 & -0.18 & 0 & - & - & - & - & - & - \\
\hline GeoB5546-2 & 27.54 & -13.74 & 1072 & Alkenone & 3 & - & - & - & - & - & - & 1 & - & - & - & - & - & - \\
\hline RN93-PC3 & 27.68 & 126.42 & 1292 & Foram & 1 & 28.08 & - & - & -0.10 & -0.30 & -0.20 & 1 & 28.08 & 0.00 & - & -0.10 & -0.30 & -0.20 \\
\hline GeoB5844-2 & 27.71 & 34.68 & 963 & Alkenone & 5 & - & - & - & - & - & - & 3 & - & - & - & - & - & - \\
\hline A7 & 27.82 & 126.97 & 1264 & Foram & 7 & 28.41 & 0.31 & 0.12 & 0.23 & 0.03 & 0.13 & 4 & 28.46 & 0.20 & 0.09 & 0.28 & 0.08 & 0.18 \\
\hline A7 & 27.82 & 126.98 & 1264 & $\mathrm{Mg} / \mathrm{Ca}$ & 7 & 25.96 & 1.49 & 0.59 & -2.22 & -2.42 & -2.32 & 4 & 26.03 & 1.19 & 0.54 & -2.15 & -2.35 & -2.25 \\
\hline RN93-PC1 & 28.56 & 127.22 & 1022 & Foram & 1 & 27.82 & - & - & -0.15 & -0.44 & -0.34 & 1 & 27.82 & 0.00 & - & -0.15 & -0.44 & -0.34 \\
\hline RN92-PC3 & 28.88 & 130.67 & 2510 & Foram & 2 & 26.99 & 1.35 & 0.96 & -1.09 & -1.32 & -1.29 & 0 & - & - & - & - & - & - \\
\hline MD02-2575 & 29.02 & -87.12 & 847 & $\mathrm{Mg} / \mathrm{Ca}$ & 5 & 26.59 & 1.59 & 0.62 & -2.21 & -2.65 & -2.99 & 2 & 27.09 & 0.39 & 0.28 & -1.73 & -2.16 & -2.49 \\
\hline GeoB6007 & 30.85 & -10.27 & 583 & Alkenone & 33 & - & - & - & - & - & - & 17 & - & - & - & - & - & - \\
\hline RN94-PC3 & 30.92 & 131.85 & 1536 & Foram & 2 & 27.80 & 0.49 & 0.34 & 0.00 & -0.27 & -0.04 & 2 & 27.80 & 0.49 & 0.35 & 0.00 & -0.27 & -0.04 \\
\hline
\end{tabular}




\begin{tabular}{|c|c|c|c|c|c|c|c|c|c|c|c|c|c|c|c|c|c|c|}
\hline ST.19 & 31.10 & 138.67 & 3336 & Alkenone & 1 & - & - & - & - & - & - & 0 & - & - & - & - & - & - \\
\hline B-3GC & 31.48 & 128.52 & 555 & Foram & 6 & 27.56 & 0.40 & 0.13 & 0.70 & 0.20 & 0.33 & 3 & 27.50 & 0.24 & 0.13 & 0.64 & 0.14 & 0.27 \\
\hline KY07-04-PC1 & 31.63 & 128.95 & 758 & $\mathrm{Mg} / \mathrm{Ca}$ & 12 & 26.29 & 2.18 & 0.67 & -0.73 & -1.07 & -0.94 & 7 & 26.17 & 1.21 & 0.63 & -0.85 & -1.20 & -1.06 \\
\hline RN92-PC4 & 31.67 & 128.72 & 710 & Foram & 3 & 26.63 & 1.67 & 0.95 & -0.22 & -0.73 & -0.60 & 1 & 27.14 & 0.00 & - & 0.28 & -0.22 & -0.09 \\
\hline ODP1012B & 32.28 & -118.38 & 1772 & Alkenone & 1 & - & - & - & - & - & - & 1 & - & - & - & - & - & - \\
\hline ST.14 & 32.67 & 138.46 & 3252 & Alkenone & 1 & - & - & - & - & - & - & 1 & - & - & - & - & - & - \\
\hline $\begin{array}{l}\text { KNR140- } \\
\text { 2_51GGC }\end{array}$ & 32.78 & -76.28 & 1790 & Foram & 3 & 27.86 & 0.27 & 0.14 & 0.06 & -0.11 & -0.54 & 1 & 27.91 & 0.00 & - & 0.11 & -0.06 & -0.49 \\
\hline ODP969E & 33.84 & 24.88 & 2201 & Alkenone & 3 & - & - & - & - & - & - & 2 & - & - & - & - & - & - \\
\hline ODP 969 & 33.84 & 24.88 & 2200 & Foram & 2 & 25.07 & 0.02 & 0.01 & 0.31 & 0.07 & -0.16 & 1 & 25.08 & 0.00 & - & 0.32 & 0.08 & -0.15 \\
\hline ODP967D & 34.07 & 32.73 & 2551 & Alkenone & 3 & - & - & - & - & - & - & 1 & - & - & - & - & - & - \\
\hline ODP1017E & 34.54 & -121.11 & 955 & Alkenone & 2 & - & - & - & - & - & - & 1 & - & - & - & - & - & - \\
\hline SSDP-102 & 34.95 & 128.88 & 40 & Alkenone & 16 & - & - & - & - & - & - & 10 & - & - & - & - & - & - \\
\hline $\begin{array}{l}\text { TTR17- } \\
\text { MS419G }\end{array}$ & 35.45 & -4.62 & 410 & Foram & 1 & 16.57 & - & - & -3.92 & -4.86 & -5.10 & 1 & 16.57 & 0.00 & - & -3.91 & -4.86 & -5.10 \\
\hline KS310 & 35.55 & -1.57 & 1900 & Foram & 1 & 23.72 & - & - & 1.37 & 0.68 & 0.48 & 0 & - & - & - & - & - & - \\
\hline MD81-LC21 & 35.66 & 26.58 & 1522 & Foram & 5 & 24.51 & 0.45 & 0.17 & 0.76 & 0.59 & -0.17 & 3 & 24.45 & 0.18 & 0.09 & 0.70 & 0.52 & -0.24 \\
\hline ODP 973 & 35.78 & 18.95 & 3695 & Foram & 2 & 25.07 & 1.75 & 1.24 & -0.18 & -0.70 & -0.98 & 1 & 24.19 & 0.00 & - & -1.06 & -1.58 & -1.86 \\
\hline ODP977 & 36.03 & -1.96 & 1984 & Alkenone & 1 & - & - & - & - & - & - & 1 & - & - & - & - & - & - \\
\hline MD95-2043 & 36.14 & -2.62 & 1841 & Alkenone & 9 & - & - & - & - & - & - & 4 & - & - & - & - & - & - \\
\hline MD 95-2043 & 36.14 & -2.62 & 1841 & Foram & 4 & 22.51 & 0.26 & 0.19 & 0.62 & -1.42 & -0.15 & 2 & 20.39 & 1.05 & 0.74 & -1.50 & -3.54 & -2.27 \\
\hline MD95-2043 & 36.14 & -2.62 & 1841 & Dinocyst & 5 & 21.11 & 3.23 & 1.19 & -0.78 & -2.82 & -1.55 & 2 & 22.36 & 0.13 & 0.09 & 0.47 & -1.57 & -0.30 \\
\hline ODP 977 & 36.19 & -1.57 & 1984 & Foram & 2 & 21.98 & 2.41 & 1.70 & -0.83 & -1.95 & -1.47 & 1 & 23.19 & 0.00 & - & 0.38 & -0.74 & -0.26 \\
\hline $\begin{array}{l}\text { ODP Leg } 161 \\
\text { Site976 HoleC }\end{array}$ & 36.21 & -4.31 & 1108 & Dinocyst & 2 & 20.62 & 0.00 & 0.00 & 0.01 & -0.49 & -1.05 & 1 & 20.62 & 0.00 & - & 0.01 & -0.49 & -1.05 \\
\hline ODP 964 & 36.26 & 17.74 & 3657 & Foram & 1 & 24.82 & - & - & -0.35 & -0.73 & -1.02 & 1 & 24.82 & 0.00 & - & -0.35 & -0.73 & -1.02 \\
\hline M39-008 & 36.38 & -7.08 & 576 & Alkenone & 2 & - & - & - & - & - & - & 1 & - & - & - & - & - & - \\
\hline GeoB5901-2 & 36.38 & -7.07 & 574 & Alkenone & 29 & - & - & - & - & - & - & 14 & - & - & - & - & - & - \\
\hline RL11 & 36.75 & 17.72 & 3376 & Alkenone & 2 & - & - & - & - & - & - & 1 & - & - & - & - & - & - \\
\hline $\begin{array}{l}\text { CH07-98- } \\
\text { GGC19 }\end{array}$ & 36.87 & -74.57 & 1049 & Alkenone & 13 & - & - & - & - & - & - & 6 & - & - & - & - & - & - \\
\hline MD04-2747 & 36.95 & 11.67 & 770 & Dinocyst & 2 & 23.59 & 0.05 & 0.03 & -0.12 & -0.87 & -1.58 & 1 & 23.57 & 0.00 & - & -0.14 & -0.90 & -1.60 \\
\hline
\end{tabular}




\begin{tabular}{|c|c|c|c|c|c|c|c|c|c|c|c|c|c|c|c|c|c|c|}
\hline $\mathrm{CQ}$ & & & & & & & & & & & & & & & & & & \\
\hline $\begin{array}{l}\text { MD04- } \\
2797 C Q\end{array}$ & 36.95 & 11.67 & 771 & Foram & 2 & 21.48 & 2.10 & 1.48 & -2.22 & -2.98 & -3.69 & 1 & 22.53 & 0.00 & - & -1.18 & -1.93 & -2.64 \\
\hline $\begin{array}{l}\text { M40-4-SL78 } \\
\text { /78MUC8 }\end{array}$ & 37.04 & 13.19 & 470 & Alkenone & 2 & - & - & - & - & - & - & 2 & - & - & - & - & - & - \\
\hline SU81-18 & 37.77 & -10.18 & 3135 & Alkenone & 2 & - & - & - & - & - & - & 0 & - & - & - & - & - & - \\
\hline MD95-2042 & 37.80 & -10.17 & 3146 & Alkenone & 3 & - & - & - & - & - & - & 1 & - & - & - & - & - & - \\
\hline MD01-2334 & 37.80 & -10.17 & 3166 & $\mathrm{Mg} / \mathrm{Ca}$ & 2 & 16.22 & 0.70 & 0.49 & -3.72 & -4.21 & -4.39 & 2 & 16.22 & 0.70 & 0.49 & -3.73 & -4.21 & -4.39 \\
\hline MD01-2443 & 37.88 & -10.18 & 2925 & Alkenone & 6 & - & - & - & - & - & - & 3 & - & - & - & - & - & - \\
\hline BS79-33 & 38.26 & 14.03 & 1282 & Alkenone & 1 & - & - & - & - & - & - & 1 & - & - & - & - & - & - \\
\hline BS79-38 & 38.41 & 13.58 & 1489 & Alkenone & 3 & - & - & - & - & - & - & 2 & - & - & - & - & - & - \\
\hline D13882 & 38.63 & -9.45 & 88 & Alkenone & 8 & - & - & - & - & - & - & 4 & - & - & - & - & - & - \\
\hline ODP 975 & 38.89 & 4.50 & 2426 & Foram & 2 & 23.11 & 0.43 & 0.30 & -1.32 & -1.78 & -1.62 & 1 & 23.32 & 0.00 & - & -1.10 & -1.57 & -1.41 \\
\hline ODP 974 & 40.35 & 12.13 & 3493 & Foram & 2 & 19.53 & 0.07 & 0.05 & -4.41 & -5.18 & -5.21 & 2 & 19.53 & 0.06 & 0.05 & -4.42 & -5.19 & -5.22 \\
\hline MD95-2040 & 40.58 & -9.86 & 2465 & Foram & 4 & 17.89 & 2.01 & 0.89 & -0.69 & -0.94 & -1.60 & 2 & 18.20 & 1.14 & 0.81 & -0.38 & -0.63 & -1.29 \\
\hline MD01-2430 & 40.80 & 27.73 & 580 & Dinocyst & 2 & 13.48 & 0.43 & 0.30 & - & -11.53 & -9.50 & 0 & - & - & - & - & - & - \\
\hline M44-KL71 & 40.84 & 27.76 & 566 & Alkenone & 5 & - & - & - & - & - & - & 3 & - & - & - & - & - & - \\
\hline AD91-17 & 40.87 & 18.64 & 844 & Alkenone & 3 & - & - & - & - & - & - & 1 & - & - & - & - & - & - \\
\hline $\mathrm{M} 40 / 480 \mathrm{SL}$ & 40.95 & 11.00 & 1881 & Foram & 2 & 19.86 & 0.07 & 0.05 & -3.37 & -4.52 & -4.50 & 1 & 19.82 & 0.00 & - & -3.41 & -4.56 & -4.54 \\
\hline ODP1019C & 41.68 & -124.93 & 980 & Alkenone & 9 & - & - & - & - & - & - & 3 & - & - & - & - & - & - \\
\hline CH69-K09 & 41.76 & -47.35 & 4100 & Foram & 3 & 15.82 & 2.14 & 1.17 & -4.00 & -5.05 & -5.58 & 2 & 16.09 & 2.14 & 1.51 & -3.73 & -4.78 & -5.31 \\
\hline IN68-9 & 41.78 & 17.90 & 1234 & Foram & 2 & 22.90 & 0.18 & 0.13 & 0.18 & -1.03 & -1.49 & 2 & 22.90 & 0.18 & 0.13 & 0.18 & -1.03 & -1.49 \\
\hline MD99-2346 & 42.07 & 4.15 & 2100 & Foram & 4 & 18.82 & 0.78 & 0.32 & -2.28 & -2.91 & -3.34 & 2 & 19.01 & 0.48 & 0.34 & -2.10 & -2.72 & -3.15 \\
\hline $\begin{array}{l}\mathrm{M} 40 / 4 \\
82-2-\mathrm{SL}\end{array}$ & 42.31 & 3.77 & 1070 & Foram & 10 & 19.00 & 1.30 & 0.40 & -1.75 & -2.65 & -3.33 & 5 & 19.08 & 1.30 & 0.51 & -1.68 & -2.56 & -3.25 \\
\hline W8709A-8TC & 42.54 & -127.68 & 3111 & Alkenone & 1 & - & - & - & - & - & - & 0 & - & - & - & - & - & - \\
\hline $\begin{array}{l}\text { OCE326- } \\
\text { GGC26 }\end{array}$ & 43.48 & -54.87 & 3975 & Alkenone & 7 & - & - & - & - & - & - & 3 & - & - & - & - & - & - \\
\hline $\begin{array}{l}\text { OCE326- } \\
\text { GGC26 }\end{array}$ & 43.48 & -54.87 & 3975 & $\mathrm{Mg} / \mathrm{Ca}$ & 1 & 13.67 & - & - & -3.14 & -3.68 & -4.76 & 1 & 13.67 & 0.00 & - & -3.14 & -3.68 & -4.76 \\
\hline $\begin{array}{l}\text { OCE326- } \\
\text { GGC30 }\end{array}$ & 43.88 & -62.80 & 250 & Alkenone & 15 & - & - & - & - & - & - & 8 & - & - & - & - & - & - \\
\hline
\end{tabular}




\begin{tabular}{|c|c|c|c|c|c|c|c|c|c|c|c|c|c|c|c|c|c|c|}
\hline $\begin{array}{l}\text { HU-90-031- } \\
044\end{array}$ & 44.49 & -55.19 & 1381 & Dinocyst & 1 & 14.84 & - & - & -0.04 & -0.85 & -1.66 & 1 & 14.84 & 0.00 & - & -0.04 & -0.85 & -1.66 \\
\hline MD01-2412 & 44.53 & 145.04 & 1225 & Alkenone & 14 & - & - & - & - & - & - & 7 & - & - & - & - & - & - \\
\hline MD-95-2033 & 44.66 & -55.62 & 1412 & Dinocyst & 4 & 14.26 & 1.75 & 0.80 & -0.40 & -1.43 & -2.24 & 2 & 13.61 & 0.35 & 0.25 & -1.05 & -2.08 & -2.89 \\
\hline $\begin{array}{l}\text { MR00-K03- } \\
\text { PC01 }\end{array}$ & 46.19 & 152.32 & 2793 & Alkenone & 4 & - & - & - & - & - & - & 1 & - & - & - & - & - & - \\
\hline $\begin{array}{l}\text { HU-2003-033- } \\
011\end{array}$ & 48.15 & -51.50 & 241 & Dinocyst & 5 & 2.11 & 1.67 & 0.81 & -7.45 & -8.09 & -9.14 & 3 & 2.15 & 1.62 & 0.81 & -7.41 & -8.05 & -9.10 \\
\hline GGC15 & 48.17 & 151.34 & 1980 & Alkenone & 1 & - & - & - & - & - & - & 0 & - & - & - & - & - & - \\
\hline MD-99-2220 & 48.60 & -68.63 & 320 & Dinocyst & 6 & 7.42 & 2.67 & 1.11 & - & -6.02 & - & 2 & 7.91 & 1.35 & 0.96 & - & -5.53 & - \\
\hline $\begin{array}{l}\text { BENGAL } \\
13078 / 16\end{array}$ & 48.83 & -16.45 & 4844 & Foram & 2 & 19.90 & 1.93 & 1.36 & 3.31 & 3.12 & 2.88 & 1 & 20.86 & 0.00 & - & 4.28 & 4.08 & 3.84 \\
\hline JT96-09 & 48.90 & -126.88 & 920 & Alkenone & 2 & - & - & - & - & - & - & 0 & - & - & - & - & - & - \\
\hline $\begin{array}{l}\text { HU-90-031- } \\
019\end{array}$ & 49.29 & -63.99 & 322 & Dinocyst & 4 & 7.99 & 0.60 & 0.28 & -3.82 & -4.75 & -5.20 & 2 & 8.07 & 0.56 & 0.40 & -3.74 & -4.67 & -5.12 \\
\hline PC4 & 49.49 & 146.13 & 664 & Alkenone & 3 & - & - & - & - & - & - & 1 & - & - & - & - & - & - \\
\hline $\begin{array}{l}\text { HU-91-045- } \\
094\end{array}$ & 50.20 & -45.69 & 3448 & Dinocyst & 2 & 9.29 & 2.20 & 1.56 & -2.25 & -2.25 & -2.20 & 1 & 10.39 & 0.00 & - & -1.15 & -1.15 & -1.10 \\
\hline PC2 & 50.40 & 148.32 & 1258 & Alkenone & 2 & - & - & - & - & - & - & 2 & - & - & - & - & - & - \\
\hline MD01-2416 & 51.00 & 168.00 & 2317 & $\mathrm{Mg} / \mathrm{Ca}$ & 12 & 4.95 & 2.35 & 0.70 & -4.21 & -4.23 & -4.62 & 4 & 4.84 & 1.10 & 0.46 & -4.32 & -4.34 & -4.73 \\
\hline $\mathrm{CH} 77-02$ & 52.07 & -36.08 & 3744 & Foram & 8 & 9.98 & 1.93 & 0.61 & -2.25 & -1.65 & -2.20 & 5 & 9.68 & 0.31 & 0.12 & -2.56 & -1.95 & -2.49 \\
\hline $\begin{array}{l}\text { HU-91-045- } \\
080\end{array}$ & 53.06 & -33.53 & 3024 & Dinocyst & 1 & 15.35 & - & - & 3.29 & 3.69 & 3.46 & 1 & 15.35 & 0.00 & - & 3.29 & 3.69 & 3.46 \\
\hline $\begin{array}{l}\text { HU-91-045- } \\
085\end{array}$ & 53.98 & -38.64 & 3603 & Dinocyst & 5 & 13.44 & 3.97 & 1.75 & 2.98 & 2.70 & 2.27 & 3 & 12.31 & 2.19 & 1.14 & 1.85 & 1.57 & 1.13 \\
\hline NEAP17K & 54.68 & -28.35 & 2734 & Foram & 9 & 12.82 & 2.74 & 0.96 & 0.54 & 0.41 & 0.33 & 4 & 13.14 & 2.59 & 1.07 & 0.86 & 0.73 & 0.65 \\
\hline NA87-22 & 55.50 & -14.07 & 2161 & Foram & 6 & 14.03 & 2.24 & 0.75 & 0.21 & 0.18 & 0.22 & 2 & 14.17 & 0.25 & 0.18 & 0.34 & 0.32 & 0.36 \\
\hline MD-99-2254 & 56.80 & -30.66 & 2440 & Dinocyst & 7 & 9.07 & 7.40 & 2.56 & -1.79 & -2.15 & -2.18 & 4 & 8.20 & 2.38 & 1.12 & -2.65 & -3.02 & -3.04 \\
\hline MD99-2251 & 57.43 & -27.90 & 2620 & $\mathrm{Mg} / \mathrm{Ca}$ & 17 & 10.17 & 4.17 & 1.14 & -1.04 & -1.41 & -1.44 & 7 & 10.86 & 2.02 & 0.73 & -0.35 & -0.72 & -0.75 \\
\hline IOW225517 & 57.67 & 7.09 & 293 & Alkenone & 11 & - & - & - & - & - & - & 5 & - & - & - & - & - & - \\
\hline IOW225514 & 57.84 & 8.70 & 420 & Alkenone & 16 & - & - & - & - & - & - & 8 & - & - & - & - & - & - \\
\hline MD-99-2227 & 58.21 & -48.37 & 3460 & Dinocyst & 4 & 9.05 & 3.94 & 1.66 & 1.72 & 2.08 & 1.77 & 2 & 9.88 & 1.70 & 1.20 & 2.56 & 2.92 & 2.61 \\
\hline
\end{tabular}




\begin{tabular}{|c|c|c|c|c|c|c|c|c|c|c|c|c|c|c|c|c|c|c|}
\hline $\begin{array}{l}\text { HU-90-013- } \\
013\end{array}$ & 58.21 & -48.31 & 3380 & Dinocyst & 5 & 8.00 & 1.54 & 0.66 & 0.67 & 1.03 & 0.72 & 3 & 8.22 & 1.06 & 0.58 & 0.90 & 1.26 & 0.94 \\
\hline $\begin{array}{l}\text { HU-84-030- } \\
021 \text { TWC }\end{array}$ & 58.37 & -57.51 & 2853 & Dinocyst & 7 & 7.67 & 3.38 & 1.25 & 1.47 & 1.65 & 1.95 & 3 & 6.91 & 2.66 & 1.34 & 0.71 & 0.89 & 1.19 \\
\hline $\begin{array}{l}\text { HU-84-030- } \\
021 \text { PC }\end{array}$ & 58.37 & -57.51 & 2853 & Dinocyst & 8 & 6.44 & 5.11 & 1.55 & 0.24 & 0.42 & 0.72 & 3 & 7.36 & 3.55 & 1.77 & 1.15 & 1.33 & 1.63 \\
\hline MD95-2015 & 58.76 & -25.96 & 2630 & Alkenone & 11 & - & - & - & - & - & - & 6 & - & - & - & - & - & - \\
\hline MD95-2015 & 58.76 & -25.96 & 2630 & Foram & 3 & 11.60 & 0.24 & 0.13 & 0.35 & -0.08 & -0.11 & 1 & 11.51 & 0.00 & - & 0.27 & -0.17 & -0.20 \\
\hline $\begin{array}{l}\text { HU-91-045- } \\
072\end{array}$ & 58.94 & -28.74 & 2237 & Dinocyst & 11 & 9.91 & 2.30 & 0.84 & -0.73 & -1.15 & -1.14 & 5 & 9.63 & 1.70 & 0.84 & -1.02 & -1.43 & -1.42 \\
\hline HM03-133-25 & 60.11 & -6.07 & 1156 & Dinocyst & 9 & 9.36 & 5.58 & 2.21 & -2.21 & -2.12 & -2.24 & 4 & 9.25 & 4.67 & 2.58 & -2.31 & -2.23 & -2.35 \\
\hline ODP984 & 61.00 & -25.00 & 1648 & $\mathrm{Mg} / \mathrm{Ca}$ & 11 & 8.55 & 1.80 & 0.58 & -2.38 & -2.48 & -2.29 & 6 & 8.75 & 0.40 & 0.45 & -2.17 & -2.28 & -2.09 \\
\hline LINK14 & 61.80 & -6.30 & 346 & Foram & 3 & 9.75 & 0.34 & 0.18 & -0.76 & -0.95 & -1.16 & 1 & 9.54 & 0.00 & - & -0.96 & -1.16 & -1.37 \\
\hline RAPID-12-1K & 62.09 & -17.82 & 1938 & $\mathrm{Mg} / \mathrm{Ca}$ & 5 & 9.90 & 2.05 & 0.79 & -1.39 & -1.39 & -1.34 & 3 & 10.35 & 1.37 & 0.69 & -0.94 & -0.94 & -0.89 \\
\hline MD99-2284 & 62.37 & -0.98 & 1500 & Foram & 8 & 10.01 & 2.35 & 0.77 & -1.22 & -1.52 & -1.45 & 4 & 9.68 & 2.14 & 0.94 & -1.56 & -1.85 & -1.79 \\
\hline MD-99-2269 & 66.64 & -20.86 & 365 & Dinocyst & 20 & 6.36 & 2.42 & 0.63 & -0.23 & -0.92 & 0.75 & 10 & 6.34 & 1.10 & 0.36 & -0.25 & -0.94 & 0.74 \\
\hline MD95-2011 & 66.97 & 7.63 & 1048 & Alkenone & 8 & - & - & - & - & - & - & 4 & - & - & - & - & - & - \\
\hline MD95-2011 & 66.97 & 7.63 & 1048 & Foram & 10 & 10.04 & 1.16 & 0.36 & -0.98 & -1.42 & -1.38 & 6 & 10.12 & 1.15 & 0.39 & -0.90 & -1.34 & -1.30 \\
\hline JR51-GC35 & 67.00 & -17.96 & 420 & Alkenone & 11 & - & - & - & - & - & - & 5 & - & - & - & - & - & - \\
\hline M23323 & 67.77 & 5.92 & 1286 & Dinocyst & 10 & 8.49 & 2.38 & 0.64 & -2.03 & -2.24 & -2.10 & 5 & 8.73 & 1.10 & 0.45 & -1.79 & -2.00 & -1.86 \\
\hline JM-96-1207 & 68.10 & -29.35 & 404 & Dinocyst & 9 & 6.70 & 2.13 & 0.69 & 5.55 & 6.91 & - & 5 & 6.91 & 1.31 & 0.48 & 5.76 & 7.12 & - \\
\hline 2005-804-006 & 68.99 & -106.57 & 118 & Dinocyst & 6 & 1.23 & 0.96 & 0.40 & - & 0.97 & - & 3 & 1.21 & 0.77 & 0.39 & - & 0.95 & - \\
\hline PL-96-112 & 71.27 & 42.61 & 286 & Dinocyst & 9 & 7.02 & 3.63 & 1.25 & 0.28 & 0.31 & 0.48 & 5 & 7.30 & 2.89 & 1.11 & 0.56 & 0.59 & 0.76 \\
\hline HLY0501-05 & 72.69 & -157.52 & 415 & Dinocyst & 6 & 2.34 & 2.38 & 0.15 & 1.94 & 2.07 & 2.23 & 2 & 2.57 & 0.36 & 0.25 & 2.16 & 2.29 & 2.46 \\
\hline 2004-804-009 & 74.19 & -81.20 & 781 & Dinocyst & 6 & 2.93 & 4.25 & 1.54 & - & 0.95 & - & 3 & 2.34 & 2.35 & 1.19 & - & 0.36 & - \\
\hline 2005-804-004 & 74.27 & -91.09 & 350 & Dinocyst & 13 & 0.21 & 1.92 & 0.48 & - & 0.15 & - & 7 & 0.03 & 0.27 & 0.10 & - & -0.03 & - \\
\hline M23258-2 & 75.00 & 13.97 & 1768 & Alkenone & 4 & - & - & - & - & - & - & 2 & - & - & - & - & - & - \\
\hline $23258-2$ & 75.00 & 13.97 & 1768 & Foram & 17 & 4.99 & 3.53 & 0.89 & -0.96 & -0.96 & -1.79 & 9 & 4.97 & 2.73 & 0.94 & -0.98 & -0.97 & -1.80 \\
\hline $\begin{array}{l}\text { HU-91-039- } \\
008\end{array}$ & 77.27 & -74.32 & 663 & Dinocyst & 4 & 2.56 & 1.15 & 0.54 & 1.51 & 2.10 & - & 4 & 2.56 & 1.15 & 0.54 & 1.51 & 2.10 & - \\
\hline
\end{tabular}


Table 4: Summary inforamtion for individual winter records

\begin{tabular}{|c|c|c|c|c|c|c|c|c|c|c|c|c|c|c|c|c|c|c|}
\hline \multicolumn{5}{|c|}{ WINTER } & \multicolumn{7}{|c|}{ 5.5-6.5 ka BP } & \multicolumn{7}{|c|}{ 5.75-6.25 ka BP } \\
\hline $\begin{array}{l}\text { 믐 } \\
\text { ப் }\end{array}$ & 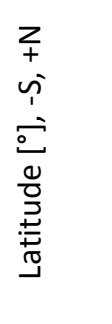 & $\begin{array}{l}山 \\
+ \\
3 \\
1 \\
1 \\
\vdots \\
0 \\
0 \\
\frac{0}{0} \\
+\frac{1}{00} \\
\frac{1}{0} \\
0\end{array}$ & 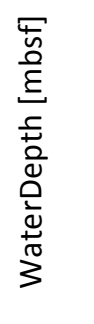 & 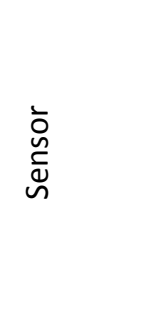 & 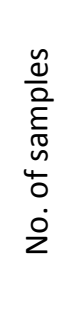 & 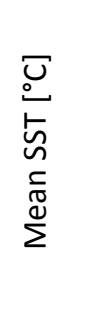 & 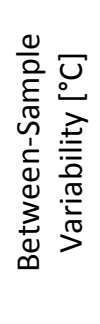 & 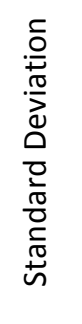 & 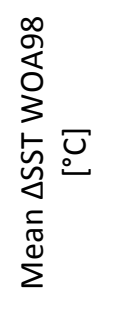 & 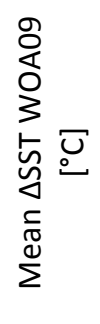 & 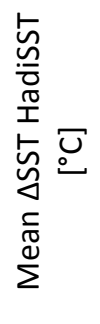 & 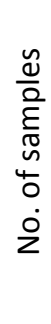 & 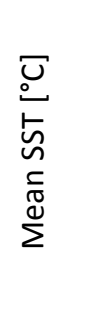 & 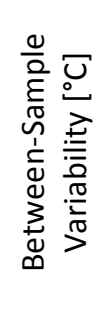 & 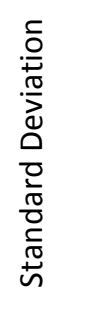 & 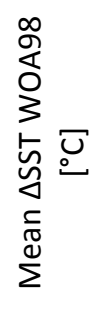 & 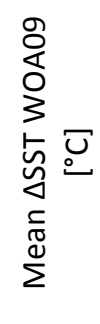 & 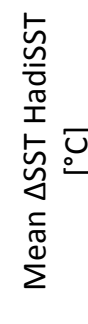 \\
\hline MD94-103 & -45.58 & 86.53 & 3560 & Alkenone & 4 & - & - & - & - & - & - & 3 & - & - & - & - & - & - \\
\hline MD97-2120 & -45.53 & 174.93 & 1210 & Alkenone & 7 & - & - & - & - & - & - & 0 & - & - & - & - & - & - \\
\hline MD97-2120 & -45.53 & 174.93 & 1210 & $\mathrm{Mg} / \mathrm{Ca}$ & 1 & - & - & - & - & - & - & 1 & - & - & - & - & - & - \\
\hline S0136-GC11 & -43.44 & 167.85 & 1556 & Alkenone & 3 & - & - & - & - & - & - & 1 & - & - & - & - & - & - \\
\hline TN057-21PC & -41.13 & 8.80 & 4981 & Alkenone & 6 & - & - & - & - & - & - & 3 & - & - & - & - & - & - \\
\hline ODP1233 & -41.01 & -74.45 & 838 & Alkenone & 3 & - & - & - & - & - & - & 2 & - & - & - & - & - & - \\
\hline GeoB3313-1 & -41.00 & -74.45 & 852 & Alkenone & 10 & - & - & - & - & - & - & 4 & - & - & - & - & - & - \\
\hline MD97-2121 & -40.38 & 177.99 & 3014 & Alkenone & 15 & - & - & - & - & - & - & 8 & - & - & - & - & - & - \\
\hline MD03-2611 & -36.73 & 136.55 & 2420 & Alkenone & 3 & - & - & - & - & - & - & 1 & - & - & - & - & - & - \\
\hline GIK17748-2 & -32.75 & -72.03 & 2545 & Alkenone & 2 & - & - & - & - & - & - & 1 & - & - & - & - & - & - \\
\hline $\begin{array}{l}\text { GIK17748-2 } \\
\text { (SO80-4) }\end{array}$ & -32.75 & -72.03 & 2545 & Foram & 2 & 11.50 & 1.18 & 0.83 & -1.72 & -2.04 & -1.83 & 1 & 10.92 & 0.00 & - & -2.31 & -2.62 & -2.41 \\
\hline GeoB7139-2 & -30.20 & -71.98 & 3270 & Alkenone & 2 & - & - & - & - & - & - & 1 & - & - & - & - & - & - \\
\hline GeoB7139-2 & -30.20 & -71.98 & 3269 & Foram & 2 & 10.92 & 0.03 & 0.02 & -2.71 & -2.64 & -2.73 & 1 & 10.94 & 0.00 & - & -2.69 & -2.62 & -2.71 \\
\hline ODP1084B & -25.51 & 13.28 & 1992 & $\mathrm{Mg} / \mathrm{Ca}$ & 8 & - & - & - & - & - & - & 3 & - & - & - & - & - & - \\
\hline GeoB7112-5 & -24.03 & -70.82 & 2507 & Foram & 3 & 10.89 & 0.53 & 0.27 & -4.46 & -4.66 & -4.27 & 2 & 10.78 & 0.35 & 0.25 & -4.59 & -4.78 & -4.39 \\
\hline GeoB1710-3 & -23.43 & 11.69 & 1045 & Alkenone & 1 & - & - & - & - & - & - & 1 & - & - & - & - & - & - \\
\hline GeoB1711-4 & -23.31 & 12.37 & 1967 & Alkenone & 1 & - & - & - & - & - & - & 1 & - & - & - & - & - & - \\
\hline GeoB1712-4 & -23.26 & 12.81 & 998 & Alkenone & 2 & - & - & - & - & - & - & 2 & - & - & - & - & - & - \\
\hline MD97-2125 & -22.57 & 161.73 & 1684 & $\mathrm{Mg} / \mathrm{Ca}$ & 2 & - & - & - & - & - & - & 0 & - & - & - & - & - & - \\
\hline MD79257 & -20.40 & 36.33 & 1262 & Alkenone & 2 & - & - & - & - & - & - & 1 & - & - & - & - & - & - \\
\hline
\end{tabular}




\begin{tabular}{|c|c|c|c|c|c|c|c|c|c|c|c|c|c|c|c|c|c|c|}
\hline GeoB1023-5 & -17.16 & 11.01 & 1978 & Alkenone & 3 & - & - & - & - & - & - & 1 & - & - & - & - & - & - \\
\hline MD01-2378 & -13.08 & 121.79 & 1783 & $\mathrm{Mg} / \mathrm{Ca}$ & 8 & - & - & - & - & - & - & 4 & - & - & - & - & - & - \\
\hline ODP1078C & -11.92 & 13.40 & 426 & Alkenone & 3 & - & - & - & - & - & - & 1 & - & - & - & - & - & - \\
\hline MD05-2928 & -11.29 & 148.86 & 2250 & Alkenone & 3 & - & - & - & - & - & - & 1 & - & - & - & - & - & - \\
\hline MD98-2170 & -10.59 & 125.39 & 832 & $\mathrm{Mg} / \mathrm{Ca}$ & 4 & - & - & - & - & - & - & 1 & - & - & - & - & - & - \\
\hline MD98-2165 & -9.65 & 118.34 & 2100 & $\mathrm{Mg} / \mathrm{Ca}$ & 5 & - & - & - & - & - & - & 2 & - & - & - & - & - & - \\
\hline SO139-74KL & -6.54 & 103.83 & 1690 & Alkenone & 24 & - & - & - & - & - & - & 12 & - & - & - & - & - & - \\
\hline GeoB10038-4 & -5.94 & 103.25 & 1819 & Alkenone & 2 & - & - & - & - & - & - & 1 & - & - & - & - & - & - \\
\hline GeoB10038-4 & -5.93 & 103.25 & 1819 & $\mathrm{Mg} / \mathrm{Ca}$ & 1 & - & - & - & - & - & - & 1 & - & - & - & - & - & - \\
\hline GeoB6518-1 & -5.59 & 11.22 & 962 & Alkenone & 6 & - & - & - & - & - & - & 2 & - & - & - & - & - & - \\
\hline $\mathrm{RC} 24-16$ & -5.04 & -10.19 & 3543 & Foram & 2 & 24.04 & 0.09 & 0.06 & 0.39 & 0.06 & -0.05 & 0 & - & - & - & - & - & - \\
\hline MD98-2176 & -5.00 & 133.45 & 2382 & $\mathrm{Mg} / \mathrm{Ca}$ & 24 & - & - & - & - & - & - & 11 & - & - & - & - & - & - \\
\hline MD98-2162 & -4.69 & 117.90 & 1855 & $\mathrm{Mg} / \mathrm{Ca}$ & 2 & - & - & - & - & - & - & 1 & - & - & - & - & - & - \\
\hline GeoB 3129-1 & -4.61 & -36.64 & 830 & Alkenone & 3 & - & - & - & - & - & - & 1 & - & - & - & - & - & - \\
\hline $\begin{array}{l}\text { GeoB3129/ } \\
3911\end{array}$ & -4.61 & -36.64 & 830 & $\mathrm{Mg} / \mathrm{Ca}$ & 3 & - & - & - & - & - & - & 1 & - & - & - & - & - & - \\
\hline GeoB3910-2 & -4.02 & -36.35 & 2362 & Alkenone & 2 & - & - & - & - & - & - & 1 & - & - & - & - & - & - \\
\hline V19-30 & -3.38 & -83.52 & 3091 & Alkenone & 1 & - & - & - & - & - & - & 1 & - & - & - & - & - & - \\
\hline V19-28 & -2.51 & -84.65 & 2720 & Alkenone & 1 & - & - & - & - & - & - & 1 & - & - & - & - & - & - \\
\hline V19-28 & -2.37 & -84.65 & 2720 & $\mathrm{Mg} / \mathrm{Ca}$ & 1 & - & - & - & - & - & - & 1 & - & - & - & - & - & - \\
\hline GeoB10029-4 & -1.50 & 100.13 & 772 & $\mathrm{Mg} / \mathrm{Ca}$ & 2 & - & - & - & - & - & - & 2 & - & - & - & - & - & - \\
\hline V21-30 & -1.22 & -89.68 & 617 & Alkenone & 3 & - & - & - & - & - & - & 1 & - & - & - & - & - & - \\
\hline V21-30 & -1.22 & -89.68 & 617 & $\mathrm{Mg} / \mathrm{Ca}$ & 5 & - & - & - & - & - & - & 1 & - & - & - & - & - & - \\
\hline GIK16773-1 & -0.97 & -9.44 & 4662 & Foram & 2 & 24.03 & 0.25 & 0.17 & 0.85 & 0.51 & 0.81 & 1 & 23.91 & 0.00 & - & 0.73 & 0.39 & 0.69 \\
\hline V19-27 & -0.47 & -82.67 & 1373 & Alkenone & 1 & - & - & - & - & - & - & 1 & - & - & - & - & - & - \\
\hline ODP1240 & 0.02 & -86.45 & 2921 & $\mathrm{Mg} / \mathrm{Ca}$ & 8 & - & - & - & - & - & - & 4 & - & - & - & - & - & - \\
\hline TR163-22 & 0.52 & -92.40 & 2830 & $\mathrm{Mg} / \mathrm{Ca}$ & 3 & - & - & - & - & - & - & 2 & - & - & - & - & - & - \\
\hline $\begin{array}{l}\text { ME0005A- } \\
24 J C\end{array}$ & 1.50 & -86.49 & 2941 & Alkenone & 3 & - & - & - & - & - & - & 2 & - & - & - & - & - & - \\
\hline TR163-19 & 2.26 & -90.95 & 2348 & $\mathrm{Mg} / \mathrm{Ca}$ & 1 & - & - & - & - & - & - & 0 & - & - & - & - & - & - \\
\hline GeoB4905-4 & 2.50 & 9.39 & 1328 & Alkenone & 6 & - & - & - & - & - & - & 3 & - & - & - & - & - & - \\
\hline GeoB4905-4 & 2.50 & 9.39 & 1328 & $\mathrm{Mg} / \mathrm{Ca}$ & 9 & - & - & - & - & - & - & 6 & - & - & - & - & - & - \\
\hline
\end{tabular}




\begin{tabular}{|c|c|c|c|c|c|c|c|c|c|c|c|c|c|c|c|c|c|c|}
\hline MD03-2707 & 2.50 & 9.40 & 1295 & $\mathrm{Mg} / \mathrm{Ca}$ & 24 & - & - & - & - & - & - & 12 & - & - & - & - & - & - \\
\hline MD85674 & 3.18 & 50.43 & 4875 & Alkenone & 2 & - & - & - & - & - & - & 0 & - & - & - & - & - & - \\
\hline $\begin{array}{l}\text { KNR176- } \\
\text { JPC32 }\end{array}$ & 4.85 & -77.96 & 2200 & Alkenone & 4 & - & - & - & - & - & - & 2 & - & - & - & - & - & - \\
\hline V30-36 & 5.35 & -27.32 & 4245 & Foram & 1 & 26.82 & - & - & -0.12 & -0.10 & 0.30 & 1 & 26.82 & 0.00 & - & -0.12 & -0.10 & 0.30 \\
\hline GIK18287-3 & 5.60 & 110.60 & 598 & Foram & 3 & 26.32 & 1.97 & 1.06 & -0.53 & -0.79 & -0.36 & 1 & 27.07 & 0.00 & - & 0.21 & -0.04 & 0.39 \\
\hline $18287-3$ & 5.65 & 110.65 & 598 & Alkenone & 3 & - & - & - & - & - & - & 1 & - & - & - & - & - & - \\
\hline GIK18287-3 & 5.98 & 110.65 & 598 & Alkenone & 3 & - & - & - & - & - & - & 1 & - & - & - & - & - & - \\
\hline 17964 & 6.16 & 112.21 & 1556 & Alkenone & 2 & - & - & - & - & - & - & 0 & - & - & - & - & - & - \\
\hline MD98-2181 & 6.30 & 125.83 & 2114 & $\mathrm{Mg} / \mathrm{Ca}$ & 22 & - & - & - & - & - & - & 12 & - & - & - & - & - & - \\
\hline MD01-2390 & 6.63 & 113.40 & 1545 & Foram & 2 & 27.32 & 0.26 & 0.19 & 0.42 & 0.18 & 0.39 & 1 & 27.19 & 0.00 & - & 0.30 & 0.05 & 0.26 \\
\hline MD01-2390 & 6.64 & 113.41 & 1545 & $\mathrm{Mg} / \mathrm{Ca}$ & 3 & - & - & - & - & - & - & 1 & - & - & - & - & - & - \\
\hline $\begin{array}{l}\text { ODP1242/ME } \\
0005 \mathrm{~A}-43 \mathrm{JC}\end{array}$ & 7.86 & -83.61 & 1366 & $\mathrm{Mg} / \mathrm{Ca}$ & 9 & - & - & - & - & - & - & 4 & - & - & - & - & - & - \\
\hline MD02-2529 & 8.21 & -84.12 & 1619 & Alkenone & 3 & - & - & - & - & - & - & 2 & - & - & - & - & - & - \\
\hline MD97-2151 & 8.73 & 109.87 & 1598 & Alkenone & 10 & - & - & - & - & - & - & 5 & - & - & - & - & - & - \\
\hline MD972151 & 8.73 & 109.87 & 283 & Foram & 3 & 26.34 & 0.66 & 0.37 & -0.13 & -0.25 & 0.29 & 1 & 26.57 & 0.00 & - & 0.10 & -0.02 & 0.52 \\
\hline MD97-2141 & 8.80 & 121.30 & 3633 & $\mathrm{Mg} / \mathrm{Ca}$ & 15 & - & - & - & - & - & - & 7 & - & - & - & - & - & - \\
\hline $18252-3$ & 9.23 & 109.38 & 1273 & Alkenone & 3 & - & - & - & - & - & - & 1 & - & - & - & - & - & - \\
\hline MD01-2392 & 9.85 & 110.21 & 1966 & Alkenone & 1 & - & - & - & - & - & - & 1 & - & - & - & - & - & - \\
\hline MD77194 & 10.47 & 75.23 & 1222 & Alkenone & 2 & - & - & - & - & - & - & 1 & - & - & - & - & - & - \\
\hline PL07-39PC & 10.70 & -65.94 & 790 & $\mathrm{Mg} / \mathrm{Ca}$ & 4 & - & - & - & - & - & - & 2 & - & - & - & - & - & - \\
\hline ODP 1002C & 10.71 & -65.17 & 893 & Alkenone & 1 & - & - & - & - & - & - & 1 & - & - & - & - & - & - \\
\hline TY93-905 & 11.07 & 51.95 & 1567 & Alkenone & 7 & - & - & - & - & - & - & 4 & - & - & - & - & - & - \\
\hline VM28-122 & 11.57 & -78.42 & 3623 & $\mathrm{Mg} / \mathrm{Ca}$ & 1 & - & - & - & - & - & - & 1 & - & - & - & - & - & - \\
\hline M35003-4 & 12.08 & -61.25 & 1299 & Alkenone & 4 & - & - & - & - & - & - & 2 & - & - & - & - & - & - \\
\hline M35003-4 & 12.09 & -61.24 & 1299 & Foram & 3 & 26.61 & 0.22 & 0.11 & 0.20 & 0.13 & 0.11 & 2 & 26.55 & 0.04 & 0.04 & 0.13 & 0.07 & 0.05 \\
\hline MD972142 & 12.69 & 119.47 & 1557 & Foram & 4 & 26.26 & 1.31 & 0.55 & -0.96 & -0.85 & -0.69 & 3 & 26.21 & 1.32 & 0.67 & -1.01 & -0.90 & -0.74 \\
\hline ODP999 & 12.75 & -78.73 & 2827 & $\mathrm{Mg} / \mathrm{Ca}$ & 1 & - & - & - & - & - & - & 1 & - & - & - & - & - & - \\
\hline MD012394 & 13.78 & 110.25 & 2097 & Foram & 5 & 25.05 & 1.34 & 0.60 & -0.72 & -1.06 & 0.13 & 2 & 25.33 & 0.84 & 0.59 & -0.44 & -0.78 & 0.41 \\
\hline $74 \mathrm{KL}$ & 14.32 & 57.35 & 3212 & Alkenone & 2 & - & - & - & - & - & - & 1 & - & - & - & - & - & - \\
\hline SCS90-36 & 18.00 & 111.49 & 2050 & Alkenone & 2 & - & - & - & - & - & - & 1 & - & - & - & - & - & - \\
\hline
\end{tabular}




\begin{tabular}{|c|c|c|c|c|c|c|c|c|c|c|c|c|c|c|c|c|c|c|}
\hline BOFS 31K & 19.00 & -20.17 & 3300 & Alkenone & 1 & - & - & - & - & - & - & 0 & - & - & - & - & - & - \\
\hline BOFS31K & 19.00 & -20.17 & 3300 & $\mathrm{Mg} / \mathrm{Ca}$ & 1 & - & - & - & - & - & - & 0 & - & - & - & - & - & - \\
\hline MD05-2904 & 19.45 & 116.25 & 2066 & $\mathrm{Mg} / \mathrm{Ca}$ & 4 & - & - & - & - & - & - & 2 & - & - & - & - & - & - \\
\hline ODP1145 & 19.58 & 117.63 & 3175 & $\mathrm{Mg} / \mathrm{Ca}$ & 1 & - & - & - & - & - & - & 1 & - & - & - & - & - & - \\
\hline $17938-2$ & 19.78 & 117.53 & 284 & Foram & 1 & 22.42 & - & - & -1.39 & -1.73 & -1.00 & 0 & - & - & - & - & - & - \\
\hline MD972148 & 19.80 & 117.54 & 4872 & Foram & 3 & 23.59 & 0.55 & 0.27 & -0.21 & -0.56 & 0.17 & 1 & 23.56 & 0.00 & - & -0.24 & -0.59 & 0.14 \\
\hline S093-126KL & 19.97 & 90.03 & 1253 & Alkenone & 2 & - & - & - & - & - & - & 0 & - & - & - & - & - & - \\
\hline $17940-2$ & 20.12 & 117.38 & 1727 & Alkenone & 8 & - & - & - & - & - & - & 8 & - & - & - & - & - & - \\
\hline MD97-2146 & 20.12 & 117.38 & 172 & Foram & 21 & 23.74 & 2.39 & 0.65 & 0.18 & 0.11 & 0.98 & 10 & 23.66 & 2.39 & 0.72 & 0.10 & 0.03 & 0.90 \\
\hline GeoB7926-2 & 20.22 & -18.45 & 2500 & Alkenone & 2 & - & - & - & - & - & - & 1 & - & - & - & - & - & - \\
\hline ODP658C & 20.75 & -18.58 & 2262 & Alkenone & 8 & - & - & - & - & - & - & 4 & - & - & - & - & - & - \\
\hline PC17 & 21.36 & -158.19 & 503 & Alkenone & 3 & - & - & - & - & - & - & 1 & - & - & - & - & - & - \\
\hline LAPAZ21P & 22.99 & -109.47 & 624 & Alkenone & 1 & - & - & - & - & - & - & 1 & - & - & - & - & - & - \\
\hline SO90-93KL & 23.58 & 64.22 & 1802 & Alkenone & 3 & - & - & - & - & - & - & 2 & - & - & - & - & - & - \\
\hline RN96-PC1 & 24.97 & 122.93 & 1676 & Foram & 4 & 23.44 & 1.19 & 0.90 & 0.57 & -0.49 & 2.87 & 1 & 24.58 & 0.00 & - & 1.71 & 0.65 & 4.01 \\
\hline 255 & 25.20 & 123.12 & 1575 & Foram & 5 & 24.44 & 1.95 & 0.75 & 1.93 & 2.38 & 4.46 & 3 & 24.91 & 0.94 & 0.49 & 2.40 & 2.85 & 4.93 \\
\hline RN88-PC5 & 25.26 & 125.15 & 2051 & Foram & 2 & 23.23 & 0.04 & 0.03 & 0.98 & 1.17 & 1.81 & 2 & 23.23 & 0.04 & 0.03 & 0.98 & 1.17 & 1.81 \\
\hline RN93-PC6 & 25.68 & 124.36 & 1849 & Foram & 2 & 22.94 & 0.35 & 0.25 & 1.19 & 0.97 & 1.99 & 0 & - & - & - & - & - & - \\
\hline RN93-PC4 & 26.55 & 125.07 & 1440 & Foram & 2 & 23.43 & 0.41 & 0.29 & 2.55 & 2.33 & 3.33 & 1 & 23.64 & 0.00 & - & 2.76 & 2.54 & 3.54 \\
\hline E017 & 26.57 & 126.03 & 1826 & Foram & 3 & 23.97 & 1.08 & 0.62 & 2.70 & 2.87 & 3.43 & 1 & 24.34 & 0.00 & - & 3.07 & 3.24 & 3.80 \\
\hline MD012404 & 26.65 & 125.81 & 1397 & Foram & 9 & 23.37 & 1.91 & 0.57 & 2.28 & 2.27 & 3.27 & 4 & 23.59 & 1.66 & 0.78 & 2.50 & 2.48 & 3.49 \\
\hline MD98-2193 & 27.40 & 126.27 & 1614 & Foram & 1 & 23.54 & - & - & 3.07 & 3.01 & 4.38 & 0 & - & - & - & - & - & - \\
\hline GeoB5546-2 & 27.54 & -13.74 & 1072 & Alkenone & 3 & - & - & - & - & - & - & 1 & - & - & - & - & - & - \\
\hline RN93-PC3 & 27.68 & 126.42 & 1292 & Foram & 1 & 23.31 & - & - & 3.08 & 2.78 & 4.15 & 1 & 23.31 & 0.00 & - & 3.08 & 2.78 & 4.15 \\
\hline GeoB5844-2 & 27.71 & 34.68 & 963 & Alkenone & 5 & - & - & - & - & - & - & 3 & - & - & - & - & - & - \\
\hline A7 & 27.82 & 126.97 & 1264 & Foram & 7 & 23.71 & 1.26 & 0.53 & 3.32 & 3.18 & 4.55 & 4 & 24.01 & 1.23 & 0.54 & 3.62 & 3.48 & 4.85 \\
\hline A7 & 27.82 & 126.98 & 1264 & $\mathrm{Mg} / \mathrm{Ca}$ & 7 & - & - & - & - & - & - & 4 & - & - & - & - & - & - \\
\hline RN93-PC1 & 28.56 & 127.22 & 1022 & Foram & 1 & 22.43 & - & - & 2.69 & 2.32 & 3.70 & 1 & 22.43 & 0.00 & - & 2.68 & 2.32 & 3.70 \\
\hline RN92-PC3 & 28.88 & 130.67 & 2510 & Foram & 2 & 21.12 & 0.95 & 0.67 & 0.37 & 0.21 & 0.92 & 0 & - & - & - & - & - & - \\
\hline MD02-2575 & 29.02 & -87.12 & 847 & $\mathrm{Mg} / \mathrm{Ca}$ & 5 & - & - & - & - & - & - & 2 & - & - & - & - & - & - \\
\hline RN94-PC3 & 30.92 & 131.85 & 1536 & Foram & 2 & 21.56 & 1.56 & 1.10 & -8.99 & 1.21 & 2.64 & 2 & 21.56 & 1.56 & 1.10 & 1.40 & 1.21 & 2.64 \\
\hline ST.19 & 31.10 & 138.67 & 3336 & Alkenone & 1 & - & - & - & - & - & - & 0 & - & - & - & - & - & - \\
\hline
\end{tabular}




\begin{tabular}{|c|c|c|c|c|c|c|c|c|c|c|c|c|c|c|c|c|c|c|}
\hline B-3GC & 31.48 & 128.52 & 555 & Foram & 6 & 20.40 & 0.92 & 0.33 & 2.64 & 2.59 & 4.39 & 3 & 20.26 & 0.59 & 0.30 & 2.50 & 2.45 & 4.25 \\
\hline KY07-04-PC1 & 31.63 & 128.95 & 758 & $\mathrm{Mg} / \mathrm{Ca}$ & 12 & - & - & - & - & - & - & 7 & - & - & - & - & - & - \\
\hline RN92-PC4 & 31.67 & 128.72 & 710 & Foram & 3 & 20.40 & 0.59 & 0.34 & 2.61 & 2.59 & 4.39 & 1 & 20.61 & 0.00 & - & 2.82 & 2.80 & 4.60 \\
\hline ODP1012B & 32.28 & -118.38 & 1772 & Alkenone & 1 & - & - & - & - & - & - & 1 & - & - & - & - & - & - \\
\hline ST.14 & 32.67 & 138.46 & 3252 & Alkenone & 1 & - & - & - & - & - & - & 1 & - & - & - & - & - & - \\
\hline $\begin{array}{l}\text { KNR140- } \\
2 \_51 G G C\end{array}$ & 32.78 & -76.28 & 1790 & Foram & 3 & 26.32 & 1.62 & 0.82 & 4.71 & 4.54 & 4.67 & 1 & 27.07 & 0.00 & - & 5.46 & 5.29 & 5.42 \\
\hline ODP969E & 33.84 & 24.88 & 2201 & Alkenone & 3 & - & - & - & - & - & - & 2 & - & - & - & - & - & - \\
\hline ODP 969 & 33.84 & 24.88 & 2200 & Foram & 2 & 16.40 & 0.40 & 0.28 & -0.05 & -0.02 & 0.30 & 1 & 16.60 & 0.00 & - & 0.15 & 0.18 & 0.50 \\
\hline ODP967D & 34.07 & 32.73 & 2551 & Alkenone & 3 & - & - & - & - & - & - & 1 & - & - & - & - & - & - \\
\hline ODP1017E & 34.54 & -121.11 & 955 & Alkenone & 2 & - & - & - & - & - & - & 1 & - & - & - & - & - & - \\
\hline SSDP-102 & 34.95 & 128.88 & 40 & Alkenone & 16 & - & - & - & - & - & - & 10 & - & - & - & - & - & - \\
\hline $\begin{array}{l}\text { TTR17- } \\
\text { MS419G }\end{array}$ & 35.45 & -4.62 & 410 & Foram & 1 & 11.33 & - & - & -4.18 & -4.25 & -4.14 & 1 & 11.33 & 0.00 & - & -4.18 & -4.25 & -4.14 \\
\hline KS310 & 35.55 & -1.57 & 1900 & Foram & 1 & 13.33 & - & - & -1.60 & -1.97 & -1.50 & 0 & - & - & - & - & - & - \\
\hline MD81-LC21 & 35.66 & 26.58 & 1522 & Foram & 5 & 15.93 & 0.29 & 0.13 & -0.51 & -0.30 & -0.16 & 3 & 15.84 & 0.10 & 0.05 & -0.60 & -0.39 & -0.24 \\
\hline ODP 973 & 35.78 & 18.95 & 3695 & Foram & 2 & 15.94 & 1.30 & 0.92 & 0.24 & 0.30 & 0.63 & 1 & 15.28 & 0.00 & - & -0.42 & -0.36 & -0.03 \\
\hline ODP977 & 36.03 & -1.96 & 1984 & Alkenone & 1 & - & - & - & - & - & - & 1 & - & - & - & - & - & - \\
\hline MD95-2043 & 36.14 & -2.62 & 1841 & Alkenone & 9 & - & - & - & - & - & - & 4 & - & - & - & - & - & - \\
\hline MD 95-2043 & 36.14 & -2.62 & 1841 & Foram & 4 & 14.67 & 0.20 & 0.11 & -1.33 & -0.15 & -0.32 & 2 & 14.68 & 0.18 & 0.13 & -0.43 & -0.14 & -0.31 \\
\hline MD95-2043 & 36.14 & -2.62 & 1841 & Dinocyst & 5 & 13.79 & 1.57 & 0.57 & -0.44 & -1.03 & -1.20 & 2 & 13.43 & 0.64 & 0.45 & -1.68 & -1.38 & -1.55 \\
\hline ODP 977 & 36.19 & -1.57 & 1984 & Foram & 2 & 13.84 & 1.81 & 1.28 & -0.86 & -0.98 & -0.90 & 1 & 14.75 & 0.00 & - & 0.05 & -0.07 & 0.01 \\
\hline $\begin{array}{l}\text { ODP Leg } 161 \\
\text { Site976 HoleC }\end{array}$ & 36.21 & -4.31 & 1108 & Dinocyst & 2 & 15.43 & 0.00 & 0.00 & 0.00 & -0.69 & -0.04 & 1 & 15.43 & 0.00 & - & 0.00 & -0.69 & -0.04 \\
\hline ODP 964 & 36.26 & 17.74 & 3657 & Foram & 1 & 15.69 & - & - & 0.21 & 0.42 & 0.73 & 1 & 15.69 & 0.00 & - & 0.21 & 0.42 & 0.73 \\
\hline M39-008 & 36.38 & -7.08 & 576 & Alkenone & 2 & - & - & - & - & - & - & 1 & - & - & - & - & - & - \\
\hline GeoB5901-2 & 36.38 & -7.07 & 574 & Alkenone & 29 & - & - & - & - & - & - & 14 & - & - & - & - & - & - \\
\hline RL11 & 36.75 & 17.72 & 3376 & Alkenone & 2 & - & - & - & - & - & - & 1 & - & - & - & - & - & - \\
\hline $\begin{array}{l}\text { CH07-98- } \\
\text { GGC19 }\end{array}$ & 36.87 & -74.57 & 1049 & Alkenone & 13 & - & - & - & - & - & - & 6 & - & - & - & - & - & - \\
\hline $\begin{array}{l}\text { MD04-2747 } \\
\mathrm{CQ}\end{array}$ & 36.95 & 11.67 & 770 & Dinocyst & 2 & 14.47 & 0.04 & 0.03 & -0.13 & -0.37 & -0.18 & 1 & 14.49 & 0.00 & - & -0.11 & -0.35 & -0.16 \\
\hline
\end{tabular}




\begin{tabular}{|c|c|c|c|c|c|c|c|c|c|c|c|c|c|c|c|c|c|c|}
\hline $\begin{array}{l}\text { MD04- } \\
2797 C Q\end{array}$ & 36.95 & 11.67 & 771 & Foram & 2 & 14.39 & 1.10 & 0.78 & -0.21 & -0.45 & -0.26 & 1 & 14.94 & 0.00 & - & 0.34 & 0.10 & 0.29 \\
\hline $\begin{array}{l}\text { M40-4- } \\
\text { SL78/78MUC8 }\end{array}$ & 37.04 & 13.19 & 470 & Alkenone & 2 & - & - & - & - & - & - & 2 & - & - & - & - & - & - \\
\hline SU81-18 & 37.77 & -10.18 & 3135 & Alkenone & 2 & - & - & - & - & - & - & 0 & - & - & - & - & - & - \\
\hline MD95-2042 & 37.80 & -10.17 & 3146 & Alkenone & 3 & - & - & - & - & - & - & 1 & - & - & - & - & - & - \\
\hline MD01-2334 & 37.80 & -10.17 & 3166 & $\mathrm{Mg} / \mathrm{Ca}$ & 2 & - & - & - & - & - & - & 2 & - & - & - & - & - & - \\
\hline MD01-2443 & 37.88 & -10.18 & 2925 & Alkenone & 6 & - & - & - & - & - & - & 3 & - & - & - & - & - & - \\
\hline BS79-33 & 38.26 & 14.03 & 1282 & Alkenone & 1 & - & - & - & - & - & - & 1 & - & - & - & - & - & - \\
\hline BS79-38 & 38.41 & 13.58 & 1489 & Alkenone & 3 & - & - & - & - & - & - & 2 & - & - & - & - & - & - \\
\hline D13882 & 38.63 & -9.45 & 88 & Alkenone & 8 & - & - & - & - & - & - & 4 & - & - & - & - & - & - \\
\hline ODP 975 & 38.89 & 4.50 & 2426 & Foram & 2 & 14.45 & 0.48 & 0.34 & -0.01 & -0.17 & 0.14 & 1 & 14.69 & 0.00 & - & 0.23 & 0.07 & 0.38 \\
\hline ODP 974 & 40.35 & 12.13 & 3493 & Foram & 2 & 12.35 & 0.03 & 0.02 & -1.86 & -1.88 & -1.56 & 2 & 12.35 & 0.04 & 0.03 & -1.86 & -1.88 & -1.56 \\
\hline MD95-2040 & 40.58 & -9.86 & 2465 & Foram & 4 & 13.40 & 1.50 & 0.66 & -0.63 & -0.62 & -0.45 & 2 & 13.64 & 0.93 & 0.66 & -0.39 & -0.38 & -0.21 \\
\hline MD01-2430 & 40.80 & 27.73 & 580 & Dinocyst & 2 & 4.89 & 1.06 & 0.75 & - & -9.55 & -6.05 & 0 & - & - & - & - & - & - \\
\hline M44-KL71 & 40.84 & 27.76 & 566 & Alkenone & 5 & - & - & - & - & - & - & 3 & - & - & - & - & - & - \\
\hline AD91-17 & 40.87 & 18.64 & 844 & Alkenone & 3 & - & - & - & - & - & - & 1 & - & - & - & - & - & - \\
\hline $\mathrm{M} 40 / 480 \mathrm{SL}$ & 40.95 & 11.00 & 1881 & Foram & 2 & 12.34 & 0.02 & 0.01 & -1.55 & -1.68 & -1.48 & 1 & 12.33 & 0.00 & - & -1.57 & -1.69 & -1.49 \\
\hline ODP1019C & 41.68 & -124.93 & 980 & Alkenone & 9 & - & - & - & - & - & - & 3 & - & - & - & - & - & - \\
\hline CH69-K09 & 41.76 & -47.35 & 4100 & Foram & 3 & 10.10 & 1.24 & 0.81 & -2.46 & -2.68 & -2.04 & 2 & 10.26 & 1.52 & 1.07 & -2.30 & -2.52 & -1.88 \\
\hline IN68-9 & 41.78 & 17.90 & 1234 & Foram & 2 & 13.86 & 0.04 & 0.03 & 0.25 & 0.21 & 0.24 & 2 & 13.86 & 0.03 & 0.02 & 0.25 & 0.20 & 0.24 \\
\hline MD99-2346 & 42.07 & 4.15 & 2100 & Foram & 4 & 12.12 & 0.31 & 0.13 & -0.60 & -0.96 & -0.94 & 2 & 12.03 & 0.18 & 0.13 & -0.69 & -1.05 & -1.03 \\
\hline $\begin{array}{l}\mathrm{M} 40 / 4 \\
82-2-\mathrm{SL}\end{array}$ & 42.31 & 3.77 & 1070 & Foram & 10 & 12.11 & 0.92 & 0.25 & -0.44 & -0.98 & -0.93 & 5 & 12.19 & 0.92 & 0.33 & -0.37 & -0.89 & -0.85 \\
\hline W8709A-8TC & 42.54 & -127.68 & 3111 & Alkenone & 1 & - & - & - & - & - & - & 0 & - & - & - & - & - & - \\
\hline $\begin{array}{l}\text { OCE326- } \\
\text { GGC26 }\end{array}$ & 43.48 & -54.87 & 3975 & Alkenone & 7 & - & - & - & - & - & - & 3 & - & - & - & - & - & - \\
\hline $\begin{array}{l}\text { OCE326- } \\
\text { GGC26 }\end{array}$ & 43.48 & -54.87 & 3975 & $\mathrm{Mg} / \mathrm{Ca}$ & 1 & - & - & - & - & - & - & 1 & - & - & - & - & - & - \\
\hline $\begin{array}{l}\text { OCE326- } \\
\text { GGC30 }\end{array}$ & 43.88 & -62.80 & 250 & Alkenone & 15 & - & - & - & - & - & - & 8 & - & - & - & - & - & - \\
\hline HU-90-031- & 44.49 & -55.19 & 1381 & Dinocyst & 1 & 2.74 & 0.00 & 0.00 & -0.24 & -0.26 & -0.24 & 1 & 2.74 & 0.00 & - & -0.24 & -0.26 & -0.24 \\
\hline
\end{tabular}




\begin{tabular}{|c|c|c|c|c|c|c|c|c|c|c|c|c|c|c|c|c|c|c|}
\hline 044 & & & & & & & & & & & & & & & & & & \\
\hline MD01-2412 & 44.53 & 145.04 & 1225 & Alkenone & 14 & - & - & - & - & - & - & 7 & - & - & - & - & - & - \\
\hline MD-95-2033 & 44.66 & -55.62 & 1412 & Dinocyst & 4 & 5.12 & 0.37 & 0.17 & 2.48 & 2.12 & 2.14 & 2 & 5.05 & 0.36 & 0.26 & 2.41 & 2.05 & 2.07 \\
\hline $\begin{array}{l}\text { MR00-K03- } \\
\text { PC01 }\end{array}$ & 46.19 & 152.32 & 2793 & Alkenone & 4 & - & - & - & - & - & - & 1 & - & - & - & - & - & - \\
\hline $\begin{array}{l}\text { HU-2003-033- } \\
011\end{array}$ & 48.15 & -51.50 & 241 & Dinocyst & 5 & -0.79 & 1.89 & 1.01 & 0.13 & 0.06 & -1.22 & 3 & -0.93 & 1.93 & 1.10 & -0.01 & -0.08 & -1.36 \\
\hline GGC15 & 48.17 & 151.34 & 1980 & Alkenone & 1 & - & - & - & - & - & - & 0 & - & - & - & - & - & - \\
\hline MD-99-2220 & 48.60 & -68.63 & 320 & Dinocyst & 6 & 1.60 & 2.92 & 1.47 & - & 2.53 & - & 2 & 2.06 & 3.02 & 2.14 & - & 2.99 & - \\
\hline $\begin{array}{l}\text { BENGAL } \\
13078 / 16\end{array}$ & 48.83 & -16.45 & 4844 & Foram & 2 & 14.23 & 3.00 & 2.12 & 2.71 & 2.53 & 2.48 & 1 & 15.73 & 0.00 & - & 4.21 & 4.03 & 3.98 \\
\hline JT96-09 & 48.90 & -126.88 & 920 & Alkenone & 2 & - & - & - & - & - & - & 0 & - & - & - & - & - & - \\
\hline $\begin{array}{l}\text { HU-90-031- } \\
019\end{array}$ & 49.29 & -63.99 & 322 & Dinocyst & 4 & -0.35 & 0.19 & 0.08 & -0.08 & 0.56 & -0.04 & 2 & -0.41 & 0.06 & 0.04 & -0.14 & 0.50 & -0.10 \\
\hline PC4 & 49.49 & 146.13 & 664 & Alkenone & 3 & - & - & - & - & - & - & 1 & - & - & - & - & - & - \\
\hline $\begin{array}{l}\text { HU-91-045- } \\
094\end{array}$ & 50.20 & -45.69 & 3448 & Dinocyst & 2 & 4.30 & 0.89 & 0.63 & -1.01 & -0.97 & -0.57 & 1 & 4.75 & 0.00 & - & -0.57 & -0.52 & -0.12 \\
\hline $\mathrm{PC} 2$ & 50.40 & 148.32 & 1258 & Alkenone & 2 & - & - & - & - & - & - & 2 & - & - & - & - & - & - \\
\hline MD01-2416 & 51.00 & 168.00 & 2317 & $\mathrm{Mg} / \mathrm{Ca}$ & 12 & - & - & - & - & - & - & 4 & - & - & - & - & - & - \\
\hline $\mathrm{CH} 77-02$ & 52.07 & -36.08 & 3744 & Foram & 8 & 6.47 & 1.72 & 0.59 & -0.59 & -0.24 & -0.38 & 5 & 6.22 & 0.37 & 0.15 & -0.84 & -0.50 & -0.63 \\
\hline $\begin{array}{l}\text { HU-91-045- } \\
080\end{array}$ & 53.06 & -33.53 & 3024 & Dinocyst & 1 & 9.61 & - & - & 2.60 & 2.91 & 2.70 & 1 & 9.61 & 0.00 & - & 2.60 & 2.91 & 2.70 \\
\hline $\begin{array}{l}\text { HU-91-045- } \\
085\end{array}$ & 53.98 & -38.64 & 3603 & Dinocyst & 5 & 8.28 & 2.88 & 1.27 & 2.94 & 2.40 & 2.51 & 3 & 7.47 & 1.69 & 0.86 & 2.13 & 1.59 & 1.70 \\
\hline NEAP17K & 54.68 & -28.35 & 2734 & Foram & 9 & 8.91 & 1.50 & 0.55 & 0.80 & 0.88 & 1.08 & 4 & 9.12 & 1.50 & 0.70 & 1.02 & 1.09 & 1.29 \\
\hline NA87-22 & 55.50 & -14.07 & 2161 & Foram & 6 & 9.83 & 0.98 & 0.32 & 0.04 & -0.03 & -0.02 & 2 & 9.82 & 0.04 & 0.03 & 0.03 & -0.04 & -0.03 \\
\hline MD-99-2254 & 56.80 & -30.66 & 2440 & Dinocyst & 7 & 4.04 & 2.25 & 1.46 & -2.81 & -3.00 & -3.12 & 4 & 3.48 & 0.72 & 0.33 & -3.37 & -3.56 & -3.68 \\
\hline MD99-2251 & 57.43 & -27.90 & 2620 & $\mathrm{Mg} / \mathrm{Ca}$ & 17 & - & - & - & - & - & - & 7 & - & - & - & - & - & - \\
\hline IOW225517 & 57.67 & 7.09 & 293 & Alkenone & 11 & - & - & - & - & - & - & 5 & - & - & - & - & - & - \\
\hline IOW225514 & 57.84 & 8.70 & 420 & Alkenone & 16 & - & - & - & - & - & - & 8 & - & - & - & - & - & - \\
\hline MD-99-2227 & 58.21 & -48.37 & 3460 & Dinocyst & 4 & 2.44 & 4.01 & 1.88 & -0.72 & -0.51 & -0.56 & 2 & 2.91 & 3.61 & 2.55 & -0.24 & -0.03 & -0.09 \\
\hline HU-90-013- & 58.21 & -48.31 & 3380 & Dinocyst & 5 & 2.81 & 1.31 & 0.54 & -0.35 & -0.14 & -0.20 & 3 & 3.12 & 0.90 & 0.46 & -0.04 & 0.17 & 0.12 \\
\hline
\end{tabular}




\begin{tabular}{|c|c|c|c|c|c|c|c|c|c|c|c|c|c|c|c|c|c|c|}
\hline 013 & & & & & & & & & & & & & & & & & & \\
\hline $\begin{array}{l}\text { HU-84-030- } \\
021 \text { TWC }\end{array}$ & 58.37 & -57.51 & 2853 & Dinocyst & 7 & 3.01 & 3.10 & 0.98 & 0.06 & 1.05 & 1.83 & 0 & - & - & - & - & - & - \\
\hline $\begin{array}{l}\text { HU-84-030- } \\
021 \text { PC }\end{array}$ & 58.37 & -57.51 & 2853 & Dinocyst & 8 & 2.45 & 3.33 & 1.20 & 0.62 & 0.49 & 1.27 & 3 & 2.98 & 2.09 & 1.05 & 0.59 & 1.02 & 1.80 \\
\hline MD95-2015 & 58.76 & -25.96 & 2630 & Alkenone & 11 & - & - & - & - & - & - & 6 & - & - & - & - & - & - \\
\hline MD95-2015 & 58.76 & -25.96 & 2630 & Foram & 3 & 7.94 & 0.26 & 0.14 & 0.18 & -0.20 & -0.19 & 1 & 7.77 & 0.00 & - & 0.01 & -0.37 & -0.36 \\
\hline $\begin{array}{l}\text { HU-91-045- } \\
072\end{array}$ & 58.94 & -28.74 & 2237 & Dinocyst & 11 & 3.65 & 4.47 & 1.29 & -3.40 & -3.81 & -3.89 & 5 & 3.76 & 1.97 & 0.86 & -3.29 & -3.70 & -3.78 \\
\hline HM03-133-25 & 60.11 & -6.07 & 1156 & Dinocyst & 9 & 5.17 & 4.29 & 1.77 & -3.05 & -2.95 & -2.90 & 4 & 5.12 & 4.57 & 2.14 & -3.10 & -3.00 & -2.95 \\
\hline ODP984 & 61.00 & -25.00 & 1648 & $\mathrm{Mg} / \mathrm{Ca}$ & 11 & - & - & - & - & - & - & 6 & - & - & - & - & - & - \\
\hline LINK14 & 61.80 & -6.30 & 346 & Foram & 3 & 6.24 & 0.64 & 0.32 & -0.66 & -1.48 & -1.20 & 1 & 5.91 & 0.00 & - & -1.00 & -1.81 & -1.53 \\
\hline RAPID-12-1K & 62.09 & -17.82 & 1938 & $\mathrm{Mg} / \mathrm{Ca}$ & 5 & - & - & - & - & - & - & 3 & - & - & - & - & - & - \\
\hline MD99-2284 & 62.37 & -0.98 & 1500 & Foram & 8 & 5.85 & 2.18 & 0.71 & -0.89 & -1.11 & -1.32 & 4 & 5.54 & 2.00 & 0.92 & -1.20 & -1.42 & -1.63 \\
\hline MD-99-2269 & 66.64 & -20.86 & 365 & Dinocyst & 20 & 1.69 & 2.18 & 0.49 & -0.98 & -1.17 & -1.15 & 10 & 1.78 & 1.03 & 0.35 & -0.88 & -1.08 & -1.06 \\
\hline MD95-2011 & 66.97 & 7.63 & 1048 & Alkenone & 8 & - & - & - & - & - & - & 4 & - & - & - & - & - & - \\
\hline MD95-2011 & 66.97 & 7.63 & 1048 & Foram & 10 & 6.04 & 0.77 & 0.26 & -0.58 & -0.69 & -0.49 & 6 & 6.09 & 0.73 & 0.29 & -0.53 & -0.64 & -0.44 \\
\hline JR51-GC35 & 67.00 & -17.96 & 420 & Alkenone & 11 & - & - & - & - & - & - & 5 & - & - & - & - & - & - \\
\hline M23323 & 67.77 & 5.92 & 1286 & Dinocyst & 10 & 3.92 & 2.62 & 0.79 & -2.48 & -2.56 & -2.17 & 5 & 4.11 & 1.22 & 0.60 & -2.28 & -2.36 & -1.97 \\
\hline JM-96-1207 & 68.10 & -29.35 & 404 & Dinocyst & 9 & 0.65 & 1.82 & 0.60 & -0.07 & 1.00 & - & 5 & 0.95 & 1.16 & 0.47 & 0.24 & 1.31 & - \\
\hline 2005-804-006 & 68.99 & -106.57 & 118 & Dinocyst & 6 & -1.34 & 0.66 & 0.25 & - & -0.77 & - & 3 & -1.23 & 0.36 & 0.20 & 97.77 & -0.66 & - \\
\hline PL-96-112 & 71.27 & 42.61 & 286 & Dinocyst & 9 & 1.27 & 2.75 & 1.38 & 0.46 & 0.32 & -0.12 & 5 & 1.13 & 3.87 & 1.60 & 0.32 & 0.18 & -0.26 \\
\hline HLY0501-05 & 72.69 & -157.52 & 415 & Dinocyst & 6 & 0.10 & 4.82 & 1.82 & 1.72 & 1.56 & - & 2 & -0.36 & 1.18 & 0.84 & 1.26 & 1.10 & - \\
\hline 2004-804-009 & 74.19 & -81.20 & 781 & Dinocyst & 6 & -1.38 & 0.48 & 0.18 & - & -0.58 & - & 3 & -1.32 & 0.46 & 0.26 & 97.68 & -0.53 & - \\
\hline 2005-804-004 & 74.27 & -91.09 & 350 & Dinocyst & 13 & -1.76 & 0.52 & 0.18 & - & -0.18 & - & 7 & -1.76 & 0.25 & 0.13 & 97.24 & -0.18 & - \\
\hline M23258-2 & 75.00 & 13.97 & 1768 & Alkenone & 4 & - & - & - & - & - & - & 2 & - & - & - & - & - & - \\
\hline $23258-2$ & 75.00 & 13.97 & 1768 & Foram & 17 & 1.33 & 2.65 & 0.72 & -1.59 & -1.56 & -1.97 & 9 & 1.31 & 2.30 & 0.70 & -1.61 & -1.58 & -1.99 \\
\hline $\begin{array}{l}\text { HU-91-039- } \\
008\end{array}$ & 77.27 & -74.32 & 663 & Dinocyst & 4 & 0.87 & 2.36 & 1.13 & 2.32 & 2.63 & - & 4 & 0.87 & 2.36 & 1.13 & 2.32 & 2.63 & - \\
\hline
\end{tabular}


Table 5: Summary information for gridded reconstructions, 5.5-6.5 ka BP

\begin{tabular}{|c|c|c|c|c|c|c|c|c|c|c|c|c|c|}
\hline \multirow[b]{2}{*}{ 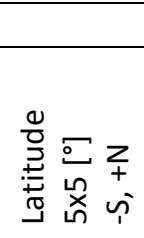 } & \multirow[b]{2}{*}{ 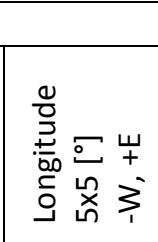 } & \multicolumn{4}{|c|}{ ANNUAL } & \multicolumn{4}{|c|}{ SUMMER } & \multicolumn{4}{|c|}{ WINTER } \\
\hline & & 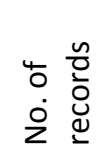 & \begin{tabular}{ll} 
& $n$ \\
0 & 0 \\
0 & \multicolumn{1}{c}{} \\
$\dot{2}$ & $n$
\end{tabular} & 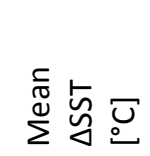 & 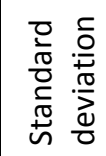 & $\begin{array}{ll}4 & 0 \\
0 & 0 \\
0 & 0 \\
2 & 0\end{array}$ & 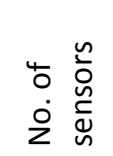 & 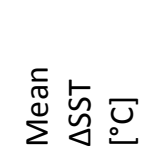 & $\begin{array}{ll}\frac{0}{0} & \frac{1}{0} \\
\frac{\pi}{0} & \frac{\pi}{\pi} \\
\frac{0}{\pi} & .0 \\
\frac{\pi}{n} & \frac{0}{0}\end{array}$ & 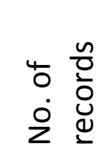 & $\begin{array}{ll} & n \\
0 & 0 \\
0 & 0 \\
\vdots & 0 \\
2 & n\end{array}$ & 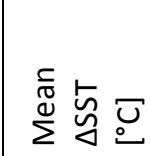 & 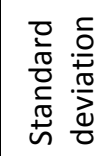 \\
\hline-45.00 & 85.00 & 1 & 1 & 0.56 & 0.54 & - & - & - & - & - & - & - & - \\
\hline-45.00 & 165.00 & 1 & 1 & 1.35 & 0.20 & - & - & - & - & - & - & - & - \\
\hline-45.00 & 175.00 & 1 & 1 & -0.47 & 0.21 & - & - & - & - & - & - & - & - \\
\hline-40.00 & -75.00 & 2 & 1 & 2.06 & 0.17 & - & - & - & - & - & - & - & - \\
\hline-40.00 & 10.00 & 1 & 1 & 6.78 & 0.72 & - & - & - & - & - & - & - & - \\
\hline-40.00 & 135.00 & 1 & 1 & 3.45 & 0.14 & - & - & - & - & - & - & - & - \\
\hline-40.00 & 175.00 & 1 & 1 & 0.81 & 0.40 & - & - & - & - & - & - & - & - \\
\hline-25.00 & -70.00 & 1 & 1 & -5.65 & 0.30 & 1 & 1 & -7.33 & 0.35 & 1 & 1 & -4.46 & 0.27 \\
\hline-25.00 & 10.00 & 1 & 1 & - & - & 1 & 1 & -3.29 & 0.74 & - & - & - & - \\
\hline-20.00 & 10.00 & 1 & 1 & 3.28 & 0.10 & - & - & - & - & - & - & - & - \\
\hline-15.00 & 10.00 & 1 & 1 & 0.07 & 0.12 & - & - & - & - & - & - & - & - \\
\hline-15.00 & 120.00 & - & - & - & - & 1 & 1 & -1.40 & 0.40 & - & - & - & - \\
\hline-10.00 & 105.00 & 1 & 1 & -0.71 & 0.17 & - & - & - & - & - & - & - & - \\
\hline-10.00 & 115.00 & - & - & - & - & 1 & 1 & -2.34 & 0.44 & - & - & - & - \\
\hline-10.00 & 125.00 & - & - & - & - & 1 & 1 & -0.15 & 0.23 & - & - & - & - \\
\hline-10.00 & 150.00 & 1 & 1 & 0.75 & 0.04 & - & - & - & - & - & - & - & - \\
\hline-5.00 & -40.00 & 1 & 1 & 0.82 & 0.08 & 1 & 1 & -0.73 & 0.32 & - & - & - & - \\
\hline-5.00 & 10.00 & 1 & 1 & 0.12 & 0.21 & - & - & - & - & - & - & - & - \\
\hline-5.00 & 130.00 & - & - & - & - & 1 & 1 & 0.15 & 0.43 & - & - & - & - \\
\hline 0.00 & -95.00 & - & - & - & - & 1 & 1 & 1.56 & 0.21 & - & - & - & - \\
\hline 0.00 & -90.00 & 1 & 1 & 1.91 & 0.20 & 1 & 1 & -3.39 & 0.18 & - & - & - & - \\
\hline 0.00 & -85.00 & 1 & 1 & -1.34 & 0.35 & 1 & 1 & 1.97 & 0.20 & - & - & - & - \\
\hline 0.00 & 10.00 & 1 & 1 & -2.49 & 0.13 & 2 & 1 & 0.52 & 0.51 & - & - & - & - \\
\hline 5.00 & -85.00 & 1 & 1 & -0.81 & 0.43 & 1 & 1 & -1.37 & 0.58 & - & - & - & - \\
\hline 5.00 & -80.00 & 1 & 1 & -0.33 & 0.16 & - & - & - & - & - & - & - & - \\
\hline
\end{tabular}




\begin{tabular}{|c|c|c|c|c|c|c|c|c|c|c|c|c|c|}
\hline 5.00 & 110.00 & 2 & 2 & -0.31 & 0.28 & 2 & 2 & -0.43 & 0.40 & 1 & 1 & -0.53 & 1.06 \\
\hline 5.00 & 125.00 & - & - & - & - & 1 & 1 & 0.58 & 0.35 & - & - & - & - \\
\hline 10.00 & -65.00 & - & - & - & - & 1 & 1 & -0.92 & 0.42 & - & - & - & - \\
\hline 10.00 & -60.00 & 2 & 2 & 0.33 & 0.12 & 1 & 1 & 0.15 & 0.07 & 1 & 1 & 0.20 & 0.11 \\
\hline 10.00 & 50.00 & 1 & 1 & -0.82 & 0.18 & - & - & - & - & - & - & - & - \\
\hline 10.00 & 110.00 & 3 & 2 & -0.44 & 0.32 & 1 & 1 & 0.05 & 0.39 & 1 & 1 & -0.13 & 0.37 \\
\hline 10.00 & 120.00 & 1 & 1 & -0.57 & 0.33 & 2 & 2 & 0.05 & 0.31 & 1 & 1 & -0.96 & 0.55 \\
\hline 15.00 & 110.00 & 1 & 1 & -0.41 & 0.39 & 1 & 1 & 0.27 & 0.20 & 1 & 1 & -0.72 & 0.60 \\
\hline 20.00 & -160.00 & 1 & 1 & 0.33 & 0.06 & - & - & - & - & - & - & - & - \\
\hline 20.00 & -20.00 & 1 & 1 & 0.89 & 0.28 & - & - & - & - & - & - & - & - \\
\hline 20.00 & 115.00 & 3 & 2 & -0.25 & 0.34 & 3 & 2 & -1.04 & 0.31 & 2 & 1 & -0.02 & 0.46 \\
\hline 25.00 & -15.00 & 1 & 1 & 1.53 & 0.20 & - & - & - & - & - & - & - & - \\
\hline 25.00 & 35.00 & 1 & 1 & 1.00 & 0.25 & - & - & - & - & - & - & - & - \\
\hline 25.00 & 65.00 & 1 & 1 & 0.97 & 0.16 & - & - & - & - & - & - & - & - \\
\hline 25.00 & 120.00 & 2 & 1 & 0.73 & 0.56 & 2 & 1 & 0.04 & 0.18 & 2 & 1 & 1.25 & 0.83 \\
\hline 25.00 & 125.00 & 3 & 1 & 1.73 & 0.41 & 4 & 2 & -0.49 & 0.30 & 3 & 1 & 2.77 & 0.57 \\
\hline 30.00 & -90.00 & - & - & - & - & 1 & 1 & -2.21 & 0.62 & - & - & - & - \\
\hline 30.00 & -75.00 & 1 & 1 & 2.59 & 0.29 & 1 & 1 & 0.06 & 0.14 & 1 & 1 & 4.71 & 0.82 \\
\hline 30.00 & -10.00 & 1 & 1 & 1.67 & 0.24 & - & - & - & - & - & - & - & - \\
\hline 30.00 & 130.00 & 2 & 1 & 1.71 & 0.40 & 3 & 2 & -0.08 & 0.58 & 2 & 1 & 2.63 & 0.34 \\
\hline 35.00 & -75.00 & 1 & 1 & -0.40 & 0.16 & - & - & - & - & - & - & - & - \\
\hline 35.00 & -10.00 & 3 & 1 & 0.97 & 0.15 & - & - & - & - & - & - & - & - \\
\hline 35.00 & -5.00 & 3 & 3 & -0.08 & 0.48 & 2 & 2 & -0.08 & 0.69 & 2 & 2 & -0.88 & 0.34 \\
\hline 35.00 & 15.00 & 1 & 1 & -2.93 & 0.71 & - & - & - & - & - & - & - & - \\
\hline 35.00 & 25.00 & 2 & 2 & -0.09 & 0.80 & 1 & 1 & 0.76 & 0.17 & 1 & 1 & -0.51 & 0.13 \\
\hline 35.00 & 30.00 & 1 & 1 & -0.89 & 0.92 & - & - & - & - & - & - & - & - \\
\hline 35.00 & 130.00 & 1 & 1 & 2.85 & 0.38 & - & - & - & - & - & - & - & - \\
\hline 40.00 & -125.00 & 1 & 1 & -1.89 & 0.35 & - & - & - & - & - & - & - & - \\
\hline 40.00 & -55.00 & 1 & 1 & 1.15 & 0.25 & - & - & - & - & - & - & - & - \\
\hline 40.00 & -50.00 & 1 & 1 & -2.99 & 0.99 & 1 & 1 & -4.00 & 1.17 & 1 & 1 & -2.46 & 0.81 \\
\hline 40.00 & -10.00 & 2 & 2 & -0.85 & 0.51 & 1 & 1 & -0.69 & 0.89 & 1 & 1 & -0.63 & 0.66 \\
\hline 40.00 & 5.00 & 2 & 1 & -1.16 & 0.25 & 2 & 1 & -2.02 & 0.36 & 2 & 1 & -0.52 & 0.19 \\
\hline
\end{tabular}




\begin{tabular}{|c|c|c|c|c|c|c|c|c|c|c|c|c|c|}
\hline 40.00 & 20.00 & 1 & 1 & -1.02 & 0.06 & - & - & - & - & - & - & - & - \\
\hline 45.00 & -65.00 & 1 & 1 & 5.30 & 0.64 & - & - & - & - & - & - & - & - \\
\hline 45.00 & -55.00 & 2 & 1 & -1.20 & 0.55 & 2 & 1 & -3.93 & 0.80 & 2 & 1 & 1.31 & 0.59 \\
\hline 45.00 & 145.00 & 1 & 1 & 6.03 & 0.50 & - & - & - & - & - & - & - & - \\
\hline 45.00 & 150.00 & 1 & 1 & 3.41 & 0.64 & - & - & - & - & - & - & - & - \\
\hline 50.00 & -70.00 & - & - & - & - & - & - & - & - & - & - & - & - \\
\hline 50.00 & -65.00 & 1 & 1 & -1.54 & 0.21 & 1 & 1 & -3.82 & 0.28 & 1 & 1 & -0.08 & 0.08 \\
\hline 50.00 & -35.00 & 1 & 1 & -1.34 & 0.60 & 1 & 1 & -2.25 & 0.61 & 1 & 1 & -0.59 & 0.59 \\
\hline 50.00 & 145.00 & 1 & 1 & 4.70 & 0.62 & - & - & - & - & - & - & - & - \\
\hline 50.00 & 165.00 & - & - & - & - & 1 & 1 & -4.21 & 0.70 & - & - & - & - \\
\hline 55.00 & -60.00 & 1 & 1 & 1.36 & 1.09 & 1 & 1 & 1.47 & 1.25 & 1 & 1 & 0.62 & 0.98 \\
\hline 55.00 & -60.00 & 1 & 1 & 0.49 & 1.34 & 1 & 1 & 0.24 & 1.55 & 1 & 1 & 0.06 & 1.20 \\
\hline 55.00 & -50.00 & 2 & 1 & 0.24 & 1.20 & 2 & 1 & 1.20 & 1.16 & 2 & 1 & -0.54 & 1.21 \\
\hline 55.00 & -40.00 & 1 & 1 & 2.93 & 1.49 & 1 & 1 & 2.98 & 1.75 & 1 & 1 & 2.94 & 1.27 \\
\hline 55.00 & -30.00 & 2 & 2 & -0.49 & 1.38 & 3 & 3 & -0.76 & 1.55 & 2 & 2 & -1.00 & 1.01 \\
\hline 55.00 & -15.00 & 1 & 1 & 0.20 & 0.47 & 1 & 1 & 0.21 & 0.75 & 1 & 1 & 0.04 & 0.32 \\
\hline 55.00 & 5.00 & 1 & 1 & 4.69 & 0.35 & - & - & - & - & - & - & - & - \\
\hline 55.00 & 10.00 & 1 & 1 & 4.28 & 0.26 & - & - & - & - & - & - & - & - \\
\hline 60.00 & -30.00 & 1 & 1 & -2.50 & 0.55 & 1 & 1 & -0.73 & 0.84 & 1 & 1 & -3.40 & 1.29 \\
\hline 60.00 & -25.00 & 2 & 2 & 1.32 & 0.30 & 2 & 2 & -1.02 & 0.36 & 1 & 1 & 0.18 & 0.14 \\
\hline 60.00 & -20.00 & - & - & - & - & 1 & 1 & -1.39 & 0.79 & - & - & - & - \\
\hline 60.00 & -5.00 & 2 & 2 & -1.82 & 1.13 & 2 & 2 & -1.48 & 1.20 & 2 & 2 & -1.86 & 1.04 \\
\hline 60.00 & 0.00 & 1 & 1 & -1.10 & 0.71 & 1 & 1 & -1.22 & 0.77 & 1 & 1 & -0.89 & 0.71 \\
\hline 65.00 & -30.00 & 1 & 1 & 2.11 & 0.56 & 1 & 1 & 5.55 & 0.69 & 1 & 1 & -0.07 & 0.60 \\
\hline 65.00 & -20.00 & 2 & 2 & 1.58 & 0.83 & 1 & 1 & -0.23 & 0.63 & 1 & 1 & -0.98 & 0.49 \\
\hline 65.00 & 5.00 & 3 & 3 & 0.49 & 0.46 & 2 & 2 & -1.50 & 0.50 & 2 & 2 & -1.53 & 0.52 \\
\hline 70.00 & -160.00 & 1 & 1 & 1.58 & 0.89 & 1 & 1 & 1.94 & 0.15 & 1 & 1 & 1.72 & 1.82 \\
\hline 70.00 & -110.00 & - & - & - & - & - & - & - & - & - & - & - & - \\
\hline 70.00 & 40.00 & 1 & 1 & 0.54 & 1.32 & 1 & 1 & 0.28 & 1.25 & 1 & 1 & 0.46 & 1.38 \\
\hline 75.00 & -80.00 & - & - & - & - & - & - & - & - & - & - & - & - \\
\hline 75.00 & -75.00 & 1 & 1 & 1.35 & 0.59 & 1 & 1 & 1.51 & 0.54 & 1 & 1 & 2.32 & 1.13 \\
\hline 75.00 & 15.00 & 2 & 2 & 0.83 & 0.54 & 1 & 1 & -0.96 & 0.89 & 1 & 1 & -1.59 & 0.72 \\
\hline
\end{tabular}



SI Figure 1: Between-sample variability in reconstructed summer sea surface temperatures (SSTS) (a) Reconstructed summer SST anomalies at individual sites with sample resolution of $<100$ years in 1000-yr window from 5.5 to $6.5 \mathrm{ka}$ BP used for mid-Holocene (MH) reconstructions. The grey bar shows the smaller 500-yr window from 5.75 to $6.25 \mathrm{ka}$ BP. (b) Variability summer SST anomalies within $6 \pm 0.5 \mathrm{ka} \mathrm{BP}$ and $6 \pm 0.25$ ka BP time windows. (c) Comparison of number of data points per record versus standard deviation at all sites.

a) WOA98-WOA09

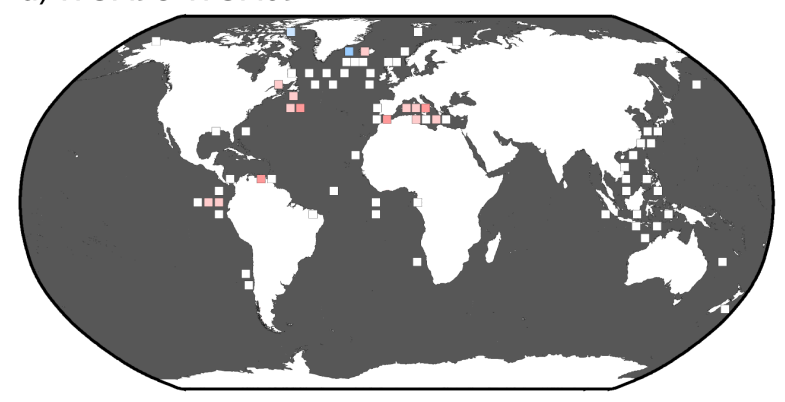

b) WOA09-HadiSST

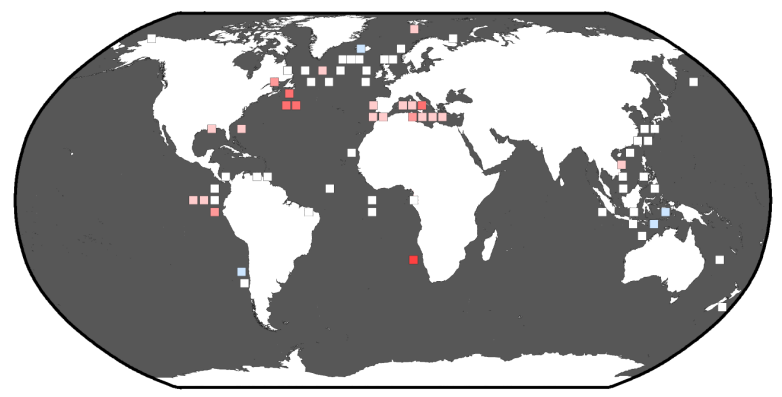

c) WOA98-GHOST
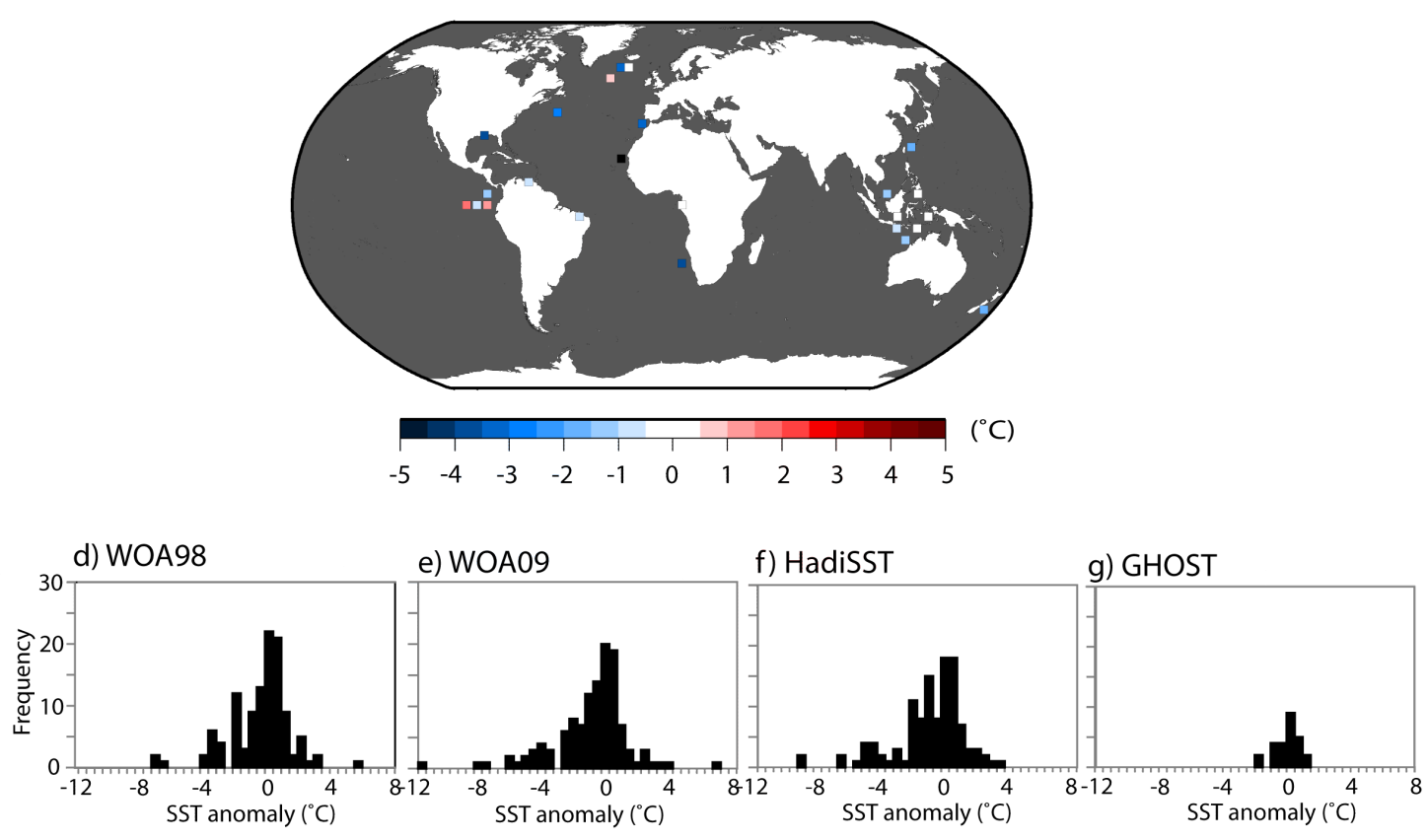
SI Figure 2: Between-sample variability in reconstructed winter sea surface temperatures (SSTS) (a) Reconstructed winter SST anomalies at individual sites with sample resolution of $<100$ years in 1000-yr window from 5.5 to $6.5 \mathrm{ka}$ BP used for mid-Holocene (MH) reconstructions. The grey bar shows the smaller 500-yr window from 5.75 to $6.25 \mathrm{ka}$ BP. (b) Variability winter SST anomalies within $6 \pm 0.5 \mathrm{ka}$ BP and $6 \pm 0.25$ ka BP time windows. (c) Comparison of number of data points per record versus standard deviation at all sites.

a) WOA98-WOA09

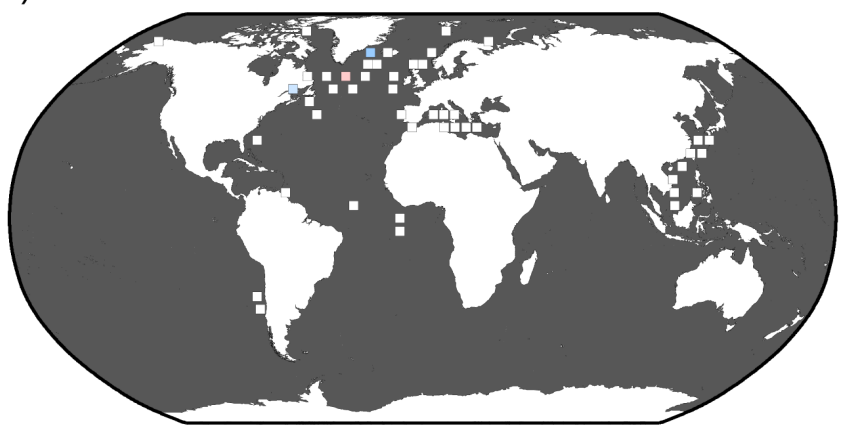

b) WOA09-HadiSST

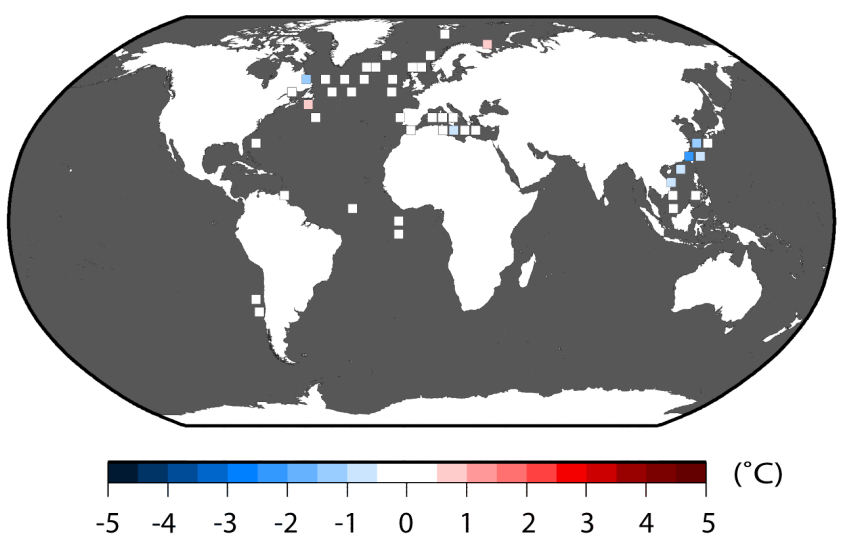

c) WOA98

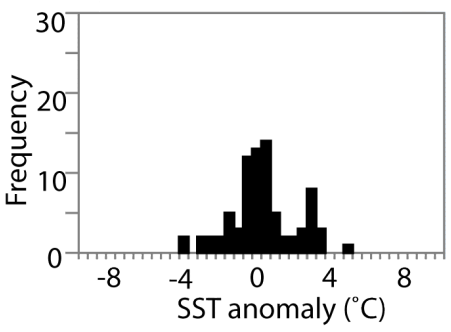

d) WOA09

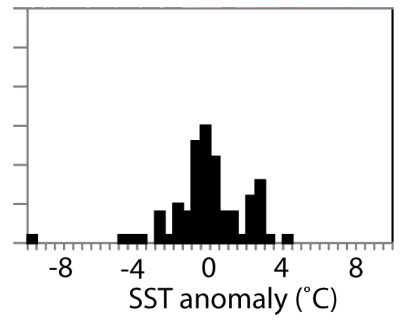

e) HadiSST

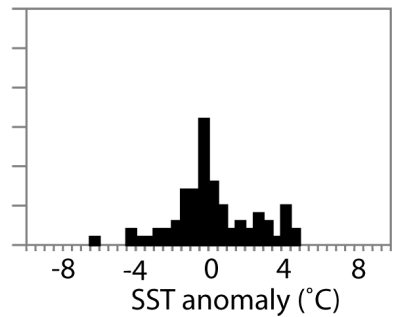


SI Figure 3: Gridded (5x5) summer sea surface temperature (SST) mid-Holocene (MH) anomalies using different modern reference climates: difference between $\mathrm{MH}$ anomalies calculated relative to (a) World Ocean Atlas data set (WOA98) and the updated version of this data set, WOA09, (b) WOA98 and Hadley Center Sea Ice and Sea Surface Temperature (HADiSST) data set, and (c) WOA98 and the Global database for alkenone and $\mathrm{Mg} / \mathrm{Ca}$-derived Holocene Sea Surface Temperature (GHOST) data set. The right hand side panels show the distribution of anomalies reconstructed using each of the reference climates: (d) WOA98, (e) WOA09, (f) HADiSST, and (g) GHOST.

a)

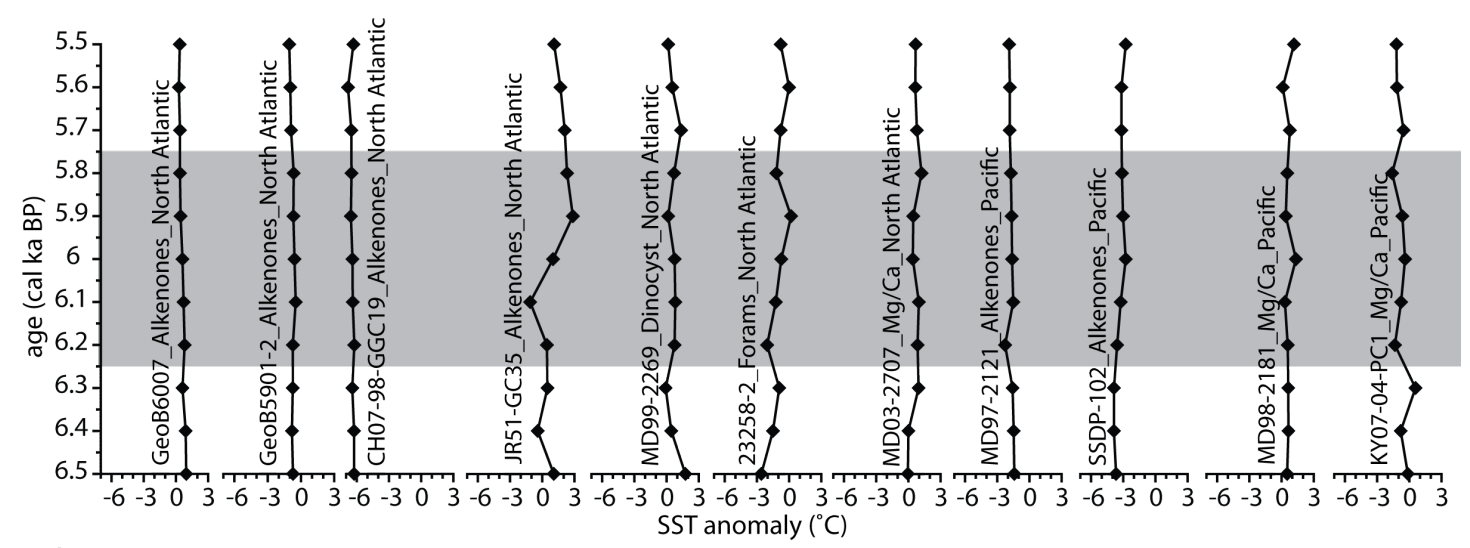

b)
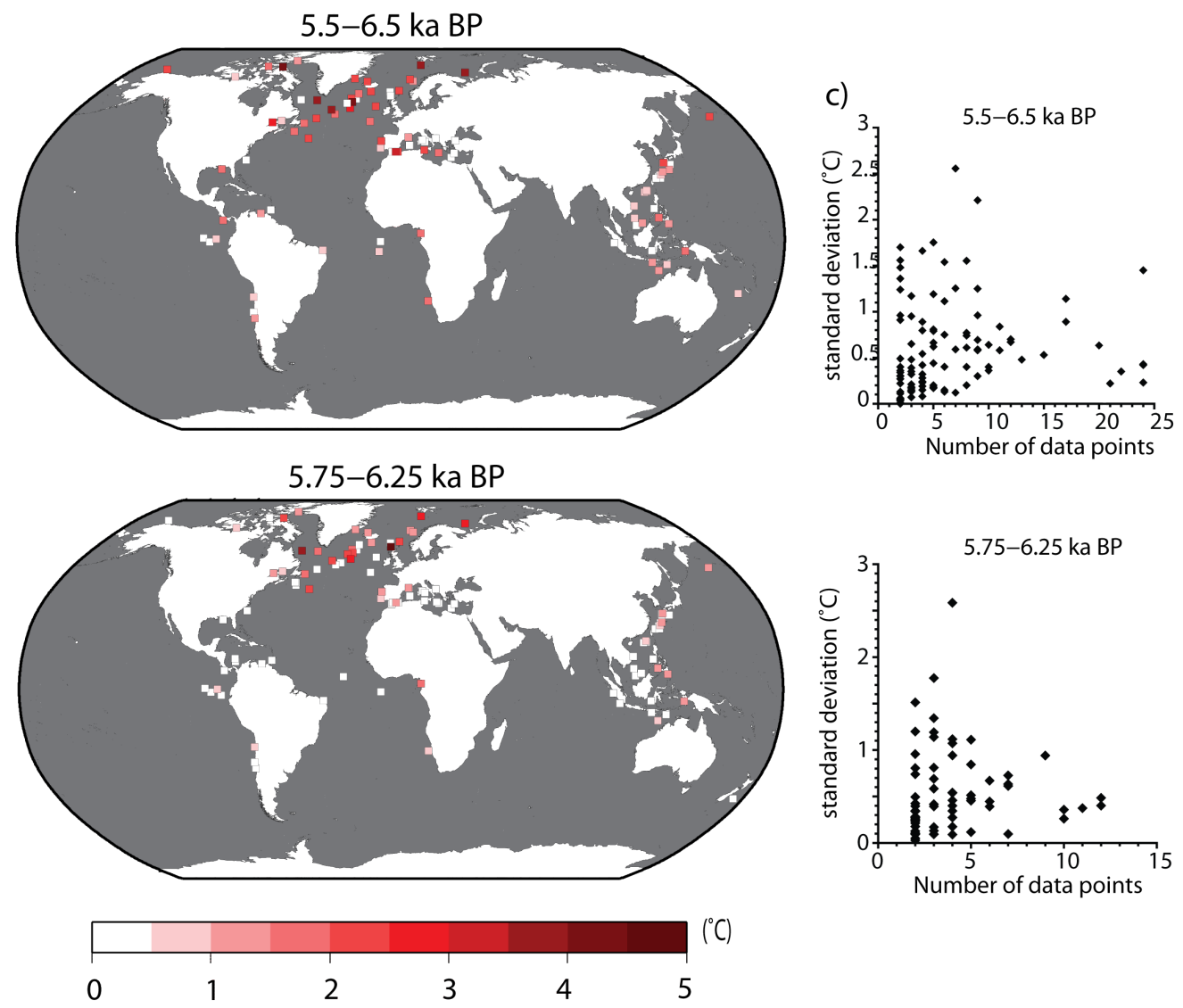
SI Figure 4: Gridded (5x5) winter sea surface temperature (SST) mid-Holocene (MH) anomalies using different modern reference climates: difference between $\mathrm{MH}$ anomalies calculated relative to (a) World Ocean Atlas data set (WOA98) and the updated version of this data set, WOA09, (b) WOA98 and Hadley Center Sea Ice and Sea Surface Temperature (HADiSST) data set. The right hand side panels show the distribution of anomalies reconstructed using each of the reference climates : (c) WOA98, (d) WOA09, (e) HADiSST.

a)

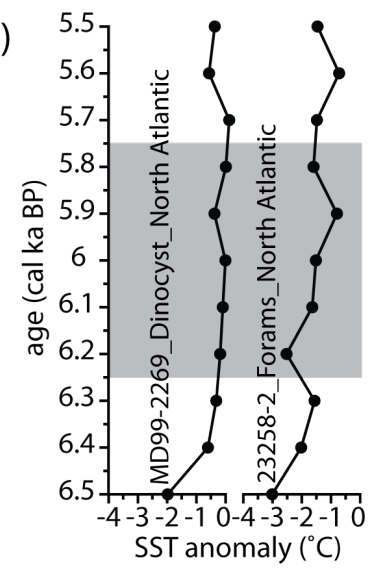

b)
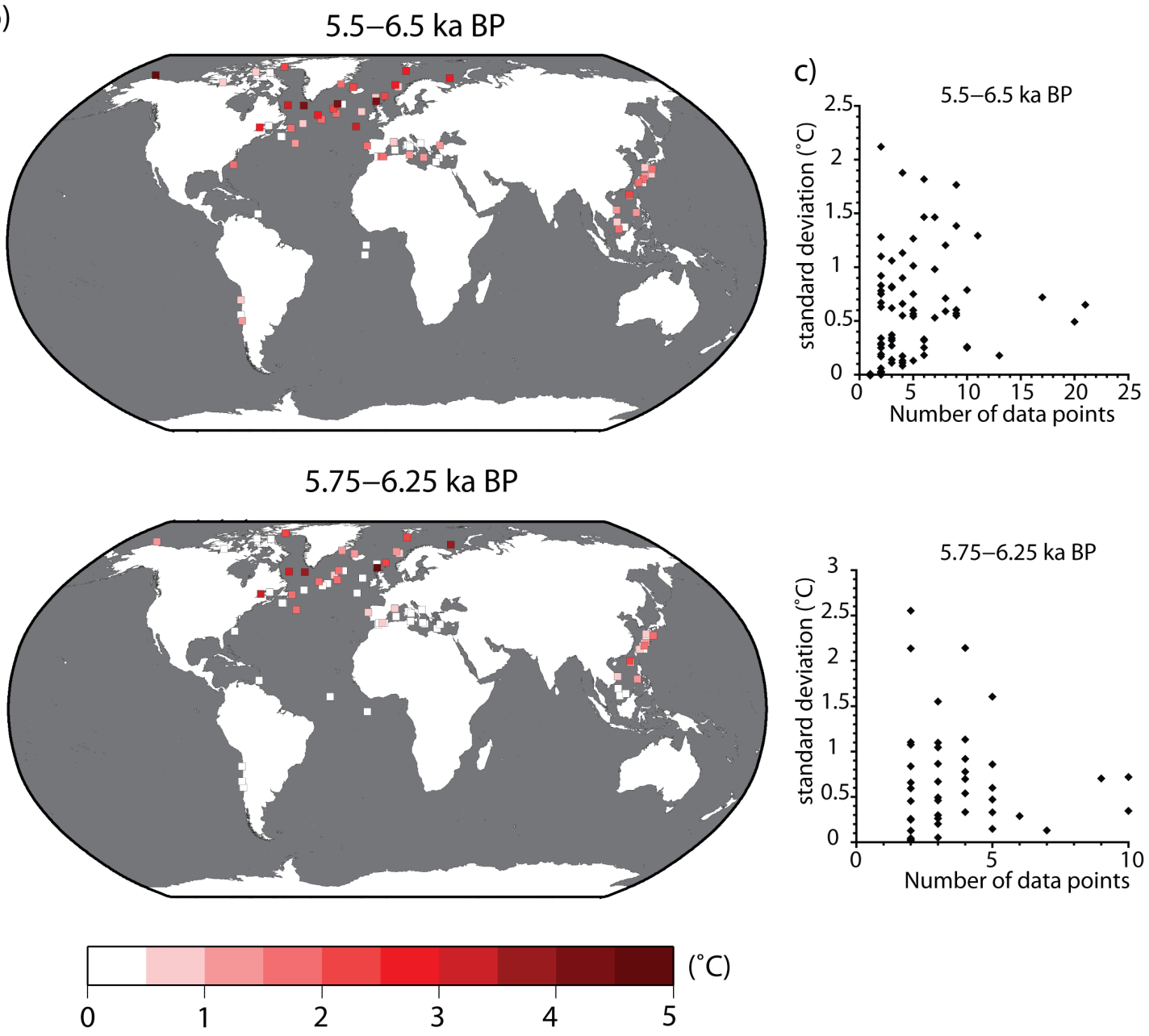
SI Figure 5: Seasonal sensor comparison using average sea surface temperature anomaly and standard deviations of individual records on a $5 \times 5^{\circ}$ grid separated by region.
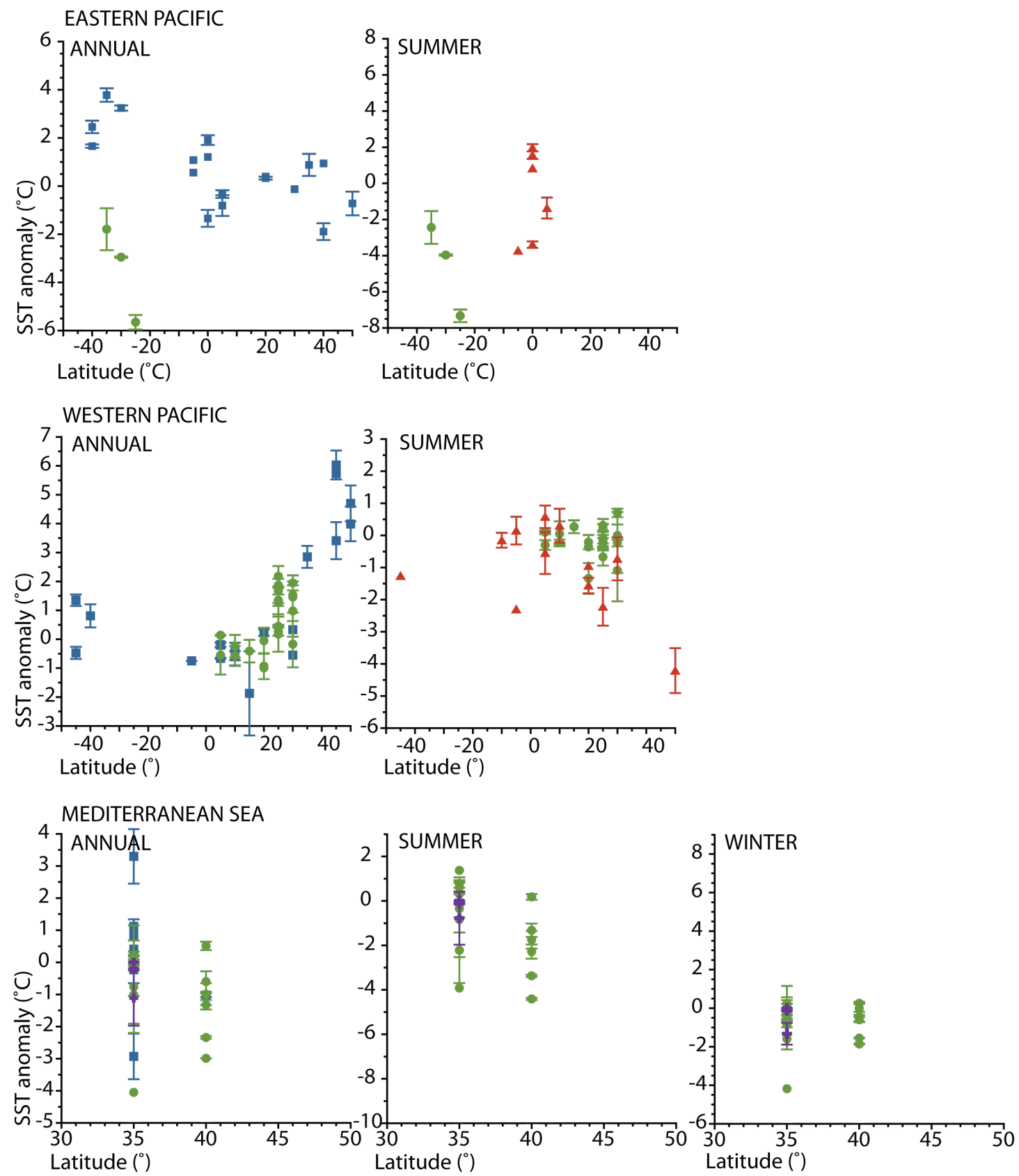

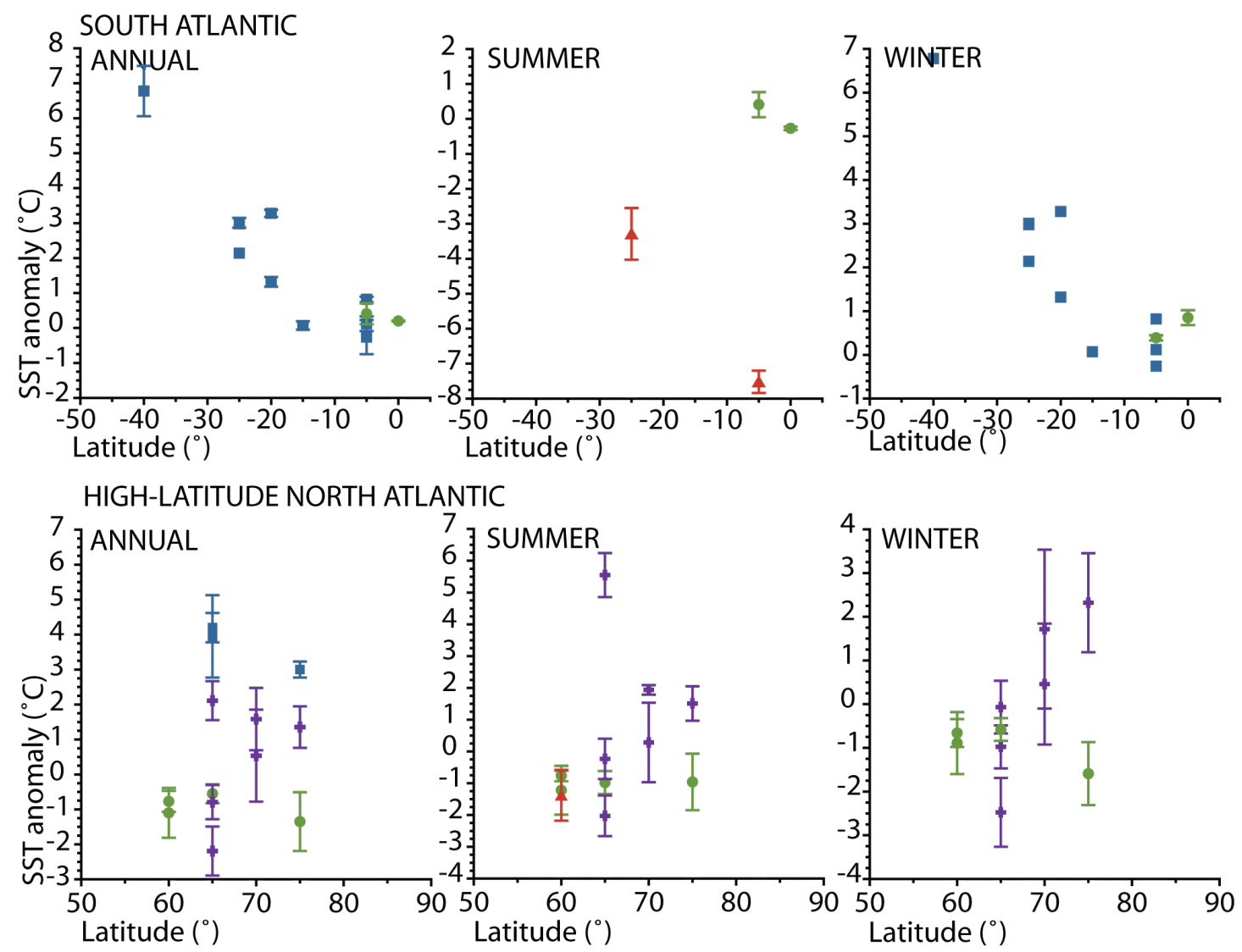

MID-LATITUDE NORTH ATLANTIC
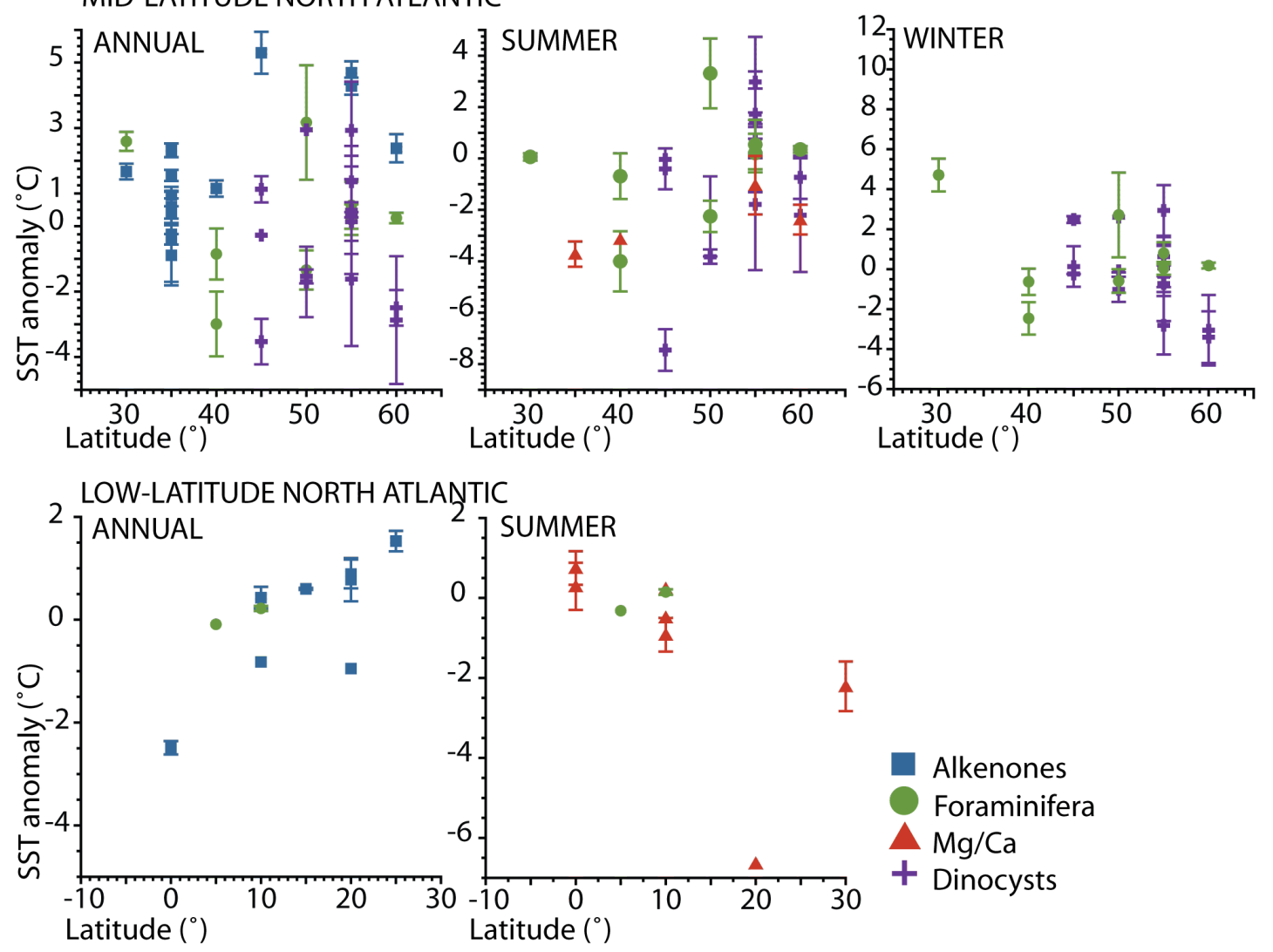


\section{References}

Anand, P., Elderfield, H., Conte, M.H., 2003. Calibration of $\mathrm{Mg} / \mathrm{Ca}$ thermometry in planktonic foraminifera from a sediment trap time series. PALEOCEANOGRAPHY 18.

Arz, H.W., Lamy, F., Patzold, J., Muller, P.J., Prins, M., 2003. Mediterranean moisture source for an early-Holocene humid period in the northern Red Sea. Science 300, 118-121.

Bard, E., Rostek, F., Sonzogni, C., 1997. Interhemispheric synchrony of the last deglaciation inferred from alkenone palaeothermometry. Nature 385, 707-710.

Bard, E., Rostek, F., Turon, J.L., Gendreau, S., 2000. Hydrological impact of Heinrich events in the subtropical northeast Atlantic. Science 289, 1321-1324.

Barker, S., Elderfield, H., 2002. Foraminiferal Calcification Response to Glacial-Interglacial Changes in Atmospheric CO2. Science 297, 833-836.

Barker, S., Greaves, M., Elderfield, H., 2003. A study of cleaning procedures used for foraminiferal Mg/Ca paleothermometry. Geochem Geophy Geosy 4.

Barron, J.A., Heusser, L., Herbert, T., Lyle, M., 2003. High-resolution climatic evolution of coastal northern California during the past 16,000 years. Paleoceanography 18.

Barrows, T.T., Lehman, S.J., Fifield, L.K., De Deckker, P., 2007. Absence of cooling in New Zealand and the adjacent ocean during the younger dryas chronozone. Science 318, 86-89.

Bendle, J.A.P., Rosell-Melé, A., 2007. High-resolution alkenone sea surface temperature variability on the North Icelandic Shelf: implications for Nordic Seas palaeoclimatic development during the Holocene. Holocene 17, 9-24.

Benway, H.M., Mix, A.C., Haley, B.A., Klinkhammer, G.P., 2006. Eastern Pacific Warm Pool paleosalinity and climate variability: 0-30 kyr. Paleoceanography 21.

Bijma, J., Altabet, M., Conte, M., Kinkel, H., Versteegh, G.J.M., Volkman, J.K., Wakeham, S.G., Weaver, P.P., 2001. Primary signal: Ecological and environmental factors - Report from Working Group 2. Geochemistry, Geophysics, Geosystems 2, 2000GC000051.

Boyle, E.A., Keigwin, L.D., 1985. Comparison of Atlantic and Pacific Paleochemical Records for the Last 215,000 Years - Changes in Deep Ocean Circulation and Chemical Inventories. Earth Planet Sc Lett 76, 135-150.

Boyle, E., Rosenthal, Y., 1996. Chemical hydrography of the South Atlantic during the last glacial maximum: Cd vs. delta C-13. South Atlantic, 423-443.

Boyle, E.A., Labeyrie, L., Duplessy, J.C., 1995. Calcitic Foraminiferal Data Confirmed by Cadmium in Aragonitic Hoeglundina - Application to the Last Glacial Maximum in the Northern Indian-Ocean. Paleoceanography 10, 881-900. 
Cacho, I., Grimalt, J. O., Canals, M., 2002. Response of the West-ern Mediterranean Sea to rapid climate variability during the last 50,000 years: a molecular biomarker approach. J. Mar. Syst. 33, 253-272.

Cacho, I., Grimalt, J.O., Canals, M., Sbaffi, L., Shackleton, N.J., Schonfeld, J., Zahn, R., 2001. Variability of the western Mediterranean Sea surface temperature during the last 25,000 years and its connection with the Northern Hemisphere climatic changes. Paleoceanography 16, 40-52.

Cacho, I., Grimalt, J.O., Pelejero, C., Canals, M., Sierro, F.J., Flores, J.A., Shackleton, N., 1999. Dansgaard-Oeschger and Heinrich Event Imprints in Alboran Sea Paleotemperatures. Paleoceanography 14, 698-705.

Calvo, E., Grimalt, J.O., Jansen, E., 2002. High resolution U(37)(K) sea surface temperature reconstruction in the Norwegian Sea during the Holocene. Quaternary Science Reviews 21, 1385-1394.

Calvo, E., Pelejero, C., De Deckker, P., Logan, G.A., 2007. Antarctic deglacial pattern in a $30 \mathrm{kyr}$ record of sea surface temperature offshore South Australia. Geophys Res Lett 34.

Came, R.E., Oppo, D.W., McManus, J.F., 2007. Amplitude and timing of temperature and salinity variability in the subpolar North Atlantic over the past 10 k.y. Geology 35, 315-318.

Chang, Y.-P., Wang, W.-L., Yokoyama, Y., Matsuzaki, H., Kawahata, H., Chen, M.-T., 2008. Millennial-scale planktic foraminifer faunal variability in the East China Sea during the Past 40000 years (IMAGES MD012404 from Okinawa Trough). Terr. Atmos. Ocean. Sci. $19,389-401$.

Chapman, M.R., Shackleton, N.J., Zhao, M., Eglinton, G., 1996. Faunal and alkenone reconstructions of subtropical North Atlantic surface hydrography and paleotemperature over the last 28 kyr. Paleoceanography 11, 343-357.

Chen, Y.-Y., Chen, M.-T., Fang, T.-S., 1999. Biogenic Sedimentation Patterns in the Northern South China Sea: An Ultrahigh-Resolution Record MD972148 of the Past 150,000 years from the IMAGES III-IPHIS Cruise. TAO 10 (1), 215-224.

Chen, M.-T., Wang, C.-H., Huang, C.-Y., Wang, P., Wang, L., Sarnthein, M., 1999. A late Quaternary planktonic foraminifer faunal record of rapid climate changes from the South China Sea. Marine Geology 156, 85-108.

Combourieu Nebout, N., Londeix, L., Baudin, F., Turon, J.L., 1999. Quaternary marine and continental paleoenvironments in the Western Mediterranean Sea (Leg 161, Site 976, Alborean Sea): Palynological evidences. Proceeding of the Ocean Drilling Project, scientific results, $161,457-468$.

Combourieu Nebout, N., Turon, J.L., Zahn, R., Capotondi, L., Londeix, L., Pahnke, K., 2002. Enhanced aridity and atmospheric high pressure stability over the western 
Mediterranean during North Atlantic cold events of the past 50000 years. Geology 30, 863-866.

Conte, M.H., Sicre, M.A., Ruhlemann, C., Weber, J.C., Schulte, S., Schulz-Bull, D., Blanz, T., 2006. Global temperature calibration of the alkenone unsaturation index UK'37 in surface waters and comparison with surface sediments. Geochem Geophy Geosy 7.

de Abreu, L., Shackleton, N.J., Schonfeld, J., Hall, M., Chapman, M., 2003. Millennial-scale oceanic climate variability off the Western Iberian margin during the last two glacial periods. Marine Geology 196, 1-20.

De Rijk, S., Hayes, A., Rohling, E.J., 1999. Eastern Mediterranean sapropel S1 interruption: an expression of the onset of climatic deterioration around 7 ka BP. Marine Geology 153, 337-343.

de Vernal, A., Eynaud, F., Henry, M., Hillaire-Marcel, C., Londeix, L., Mangin, S., Matthiessen, J., Marret, F., Radi, T., Rochon, A., Solignac, S., Turon, J.L., 2005. Reconstruction of seasurface conditions at middle to high latitudes of the Northern Hemisphere during the Last Glacial Maximum (LGM) based on dinoflagellate cyst assemblages. Quaternary Science Reviews 24, 897-924.

de Vernal, A., Hillaire-Marcel, C., 2000. Sea-ice, sea surface salinity and the halo/thermocline structure in the northern North Atlantic: modern versus full glacial conditions. Quaternary Science Reviews 19,65-85.

de Vernal, A., Hillaire-Marcel, C., 2006. Provincialism in trends and high frequency changes in the northwest North Atlantic during the Holocene. Global and Planetary Change 54, 263290.

de Vernal., A., Hillaire-Marcel, C., Rochon, A., Fréchette, B., Henry, M., Solignac, S., Bonnet, S., 2013a. Dinocyst-based reconstructions of sea ice cover concentration during the Holocene in the Arctic Ocean, the northern North Atlantic and its adjacent seas. Quaternary Science Reviews, in press. DOI:10.1016/j.quascirev.2013.07.006.

de Vernal, A., Rochon, A., Fréchette, B., Henry, M., Radi, T., Solignac, 2013b. Reconstructing past sea ice cover of the Northern Hemisphere from dinocyst assemblages: status of the approach. Quaternary Science Reviews, in press.

de Vernal, A., Rosell-Melé, A., Kucera, M., Hillaire-Marcel, C., Eynaud, F., Weinelt, M., Dokken, T., Kageyama, M., 2006. Testing the validity of proxies for the reconstruction of LGM sea surface conditions in the northern North Atlantic. Quaternary Science Reviews 25, 28202834.

Dekens, P.S., Lea, D.W., Pak, D.K., Spero, H.J., 2002. Core top calibration of $\mathrm{Mg} / \mathrm{Ca}$ in tropical foraminifera: Refining paleotemperature estimation. Geochem. Geophys. Geosyst. 3. 
deMenocal, P., Ortiz, J., Guilderson, T., Sarnthein, M., 2000. Coherent high- and low-latitude climate variability during the holocene warm period. Science 288, 2198-2202.

Duplessy, J.C., Labeyrie, L., Arnold, M., Paterne, M., Duprat, J., Vanweering, T.C.E., 1991. Changes in Surface Salinity of the North-Atlantic Ocean during the Last Deglaciation. Nature 358, 485-488.

Eggins, S., De Deckker, P., Marshall, J., 2003. Mg/Ca variation in planktonic foraminifera tests: implications for reconstructing palaeo-seawater temperature and habitat migration. Earth Planet Sc Lett 212, 291-306.

Elderfield, H., Ganssen, G., 2000. Past temperature and delta 0-18 of surface ocean waters inferred from foraminiferal $\mathrm{Mg} / \mathrm{Ca}$ ratios. Nature 405, 442-445.

Emeis, K.C., Dawson, A.G., 2003. Holocene palaeoclimate records over Europe and the North Atlantic. Holocene 13, 305-309.

Emeis, K.C., Schulz, H., Struck, U., Rossignol-Strick, M., Erlenkeuser, H., Howell, M.W., Kroon, D., Mackensen, A., Ishizuka, S., Oba, T., Sakamoto, T., Koizumi, I., 2003a. Eastern Mediterranean surface water temperatures and delta 0-18 composition during deposition of sapropels in the late Quaternary. Paleoceanography 18.

Emeis, K.C., Struck, U., Blanz, T., Kohly, A., Voss, M., 2003b. Salinity changes in the central Baltic Sea (NW Europe) over the last 10000 years. Holocene 13, 411-421.

Emeis, K.C., Struck, U., Schulz, H.M., Rosenberg, R., Bernasconi, S., Erlenkeuser, H., Sakamoto, T., Martinez-Ruiz, F., 2000. Temperature and salinity variations of Mediterranean Sea surface waters over the last 16,000 years from records of planktonic stable oxygen isotopes and alkenone unsaturation ratios. Palaeogeogr Palaeocl 158, 259-280.

Farmer, E.J., Chapman, M.R., Andrews, J.E., 2008. Centennial-scale Holocene North Atlantic surface temperatures from $\mathrm{Mg} / \mathrm{Ca}$ ratios in Globigerina bulloides. Geochem Geophy Geosy 9.

Fraile, I., Mulitza, S., Schulz, M., 2009. Modeling planktonic foraminiferal seasonality: Implications for sea-surface temperature reconstructions. Marine Micropaleontology 72, $1-9$.

Fréchette, B., de Vernal, A., 2009. Relationship between Holocene climate variations over southern Greenland and eastern Baffin Island and synoptic circulation pattern. Climate of the Past 5, 347-359.

Giunta, S; Emeis, K.-C., Negri, A., 2001. Sea-surface temperature reconstruction of the last 16,000 years in the Eastern Mediterranean Sea. Rivista Italiana Di Paleontologia E Stratigrafia, $107,463-476$.

Greaves, M., Caillon, N., Rebaubier, H., Bartoli, G., Bohaty, S., Cacho, I., Clarke, L., Cooper, M., Daunt, C., Delaney, M., deMenocal, P., Dutton, A., Eggins, S., Elderfield, H., Garbe- 
Schoenberg, D., Goddard, E., Green, D., Groeneveld, J., Hastings, D., Hathorne, E., Kimoto, K., Klinkhammer, G., Labeyrie, L., Lea, D.W., Marchiotto, T., Martínez-Botí, M.A., Mortyn, P.G., Ni, Y., Nuernberg, D., Paradis, G., Pena, L., Quinn, T., Rosenthal, Y., Russell, A., Sagawa, T., Sosdian, S., Stott, L., Tachikawa, K., Tappa, E., Thunell, R., Wilson, P.A., 2008. Interlaboratory comparison study of calibration standards for foraminiferal $\mathrm{Mg} / \mathrm{Ca}$ thermometry. Geochemistry Geophysics Geosystems 9 (8), Q08010.

Harada, N., Ahagon, N., Sakamoto, T., Uchida, M., Ikehara, M., Shibata, Y., 2006. Rapid fluctuation of alkenone temperature in the southwestern Okhotsk Sea during the past $120 \mathrm{ky}$. Global and Planetary Change 53, 29-46.

Harada, N., Ahagon, N., Uchida, M., Murayama, M., 2004. Northward and southward migrations of frontal zones during the past $40 \mathrm{kyr}$ in the Kuroshio-Oyashio transition area. Geochem Geophy Geosy 5.

Hastings, D.W., Whitko, A., Kienast, M., Steinke, S., 2001. A comparison of three independent paleotemperature estimates from a high resolution record of deglacial SST records in the South China Sea. Eos 82, PP12B-10.

Hayes, A., Rohling, E.J., De Rijk, S., Kroon, D., Zachariasse, W.J., 1999. Mediterranean planktonic foraminiferal faunas during the last glacial cycle. Marine Geology 153, 239-252.

Herbert, T., Schuffert, J.D., 2000. 16. Alkenone unsaturation estimates of sea-surface temperatures at site 1002 over a full glacial cycle. Proceedings of the Ocean Drilling Program, Scientific Results 165.

Herbert, T.D., Schuffert, J.D., Andreasen, D., Heusser, L., Lyle, M., Mix, A., Ravelo, A.C., Stott, L.D., Herguera, J.C., 2001. Collapse of the California Current during glacial maxima linked to climate change on land. Science 293, 71-76.

Huang, C.-C., Chen, M.-T., Lee, M.-Y., Wei, K.-Y., Huang, C.-Y., 2002. Planktonic foraminifer faunal sea surface temperature records of the past two glacial terminations in the South China Sea near Wan-An Shallow (IMAGES Core MD972151). Western Pacific Earth Sciences 2 (1), 1-14.

Huang, C.Y., Wu, S.F., Zhao, M.X., Chen, M.T., Wang, C.H., Tu, X., Yuan, P.B., 1997. Surface ocean and monsoon climate variability in the South China Sea since the last glaciation. Marine Micropaleontology 32, 71-94.

Hüls, M., Zahn, R., 2000. Millennial-scale sea surface temperature variability in the western tropical North Atlantic from planktonic foraminiferal census counts. Palaeoceanography $15,659-678$.

Imbrie, J., Hays, J.D., Martinson, D.G., McIntyre, A., Mix, A.C., Morley, J.J., Pisias, N.G., Prell, W.L., Shackleton, N.J. 1984. The orbital theory of Pleistocene climate: Support from a revised 
chronology of the marine $\delta^{18} 0$ record. In: Berger, A., et al. (eds), Milankovitch and Climate. Hingham MA, pp.269-305.

Jaeschke, A., Ruhlemann, C., Arz, H., Heil, G., Lohmann, G., 2007. Coupling of millennial-scale changes in sea surface temperature and precipitation off northeastern Brazil with highlatitude climate shifts during the last glacial period. Paleoceanography 22 .

Jorissen, F.J., Asioli, A., Borsetti, A.M., Capotondi, L., de Visser, J.P., Hilgen, F.J., Rohling, E.J., van der Borg, K., Vergnaud Grazzini, C., Zachariasse, W.J., 1993. Late Quaternary central Mediterranean biochronology. Marine Micropaleontology 21, 169-189.

Kaiser, J., Lamy, F., Hebbeln, D., 2005. A 70-kyr sea surface temperature record off southern Chile (Ocean Drilling Program Site 1233). Paleoceanography 20.

Kaiser, J., Schefuss, E., Lamy, F., Mohtadi, M., Hebbeln, D., 2008. Glacial to Holocene changes in sea surface temperature and coastal vegetation in north central Chile: high versus low latitude forcing. Quaternary Science Reviews 27, 2064-2075.

Keigwin, L.D., 1998. Glacial-age hydrography of the far northwest Pacific Ocean. Paleoceanography 13, 323-339.

Keigwin, L.D., Sachs, J.P., Rosenthal, Y., Boyle, E.A., 2005. The 8200 year BP event in the slope water system, western subpolar North Atlantic. Paleoceanography 20.

Kennett, J.P., Rozo-Vera, G.A., Machain Castillo, M.L., 2000. Latest Neogene planktonic foraminiferal biostratigraphy of the California margin. In Lyle, M., Koizumi, I., Richter, C., and Moore, T.C., Jr. (Eds.), Proc. ODP, Sci. Results, 167: College Station, TX (Ocean Drilling Program), 41-62.

Kerwin, M.W., Overpeck, J.T., Webb, R.S., de Vernal, A., Rind, D.H., Healy, R.J., 1999. The role of oceanic forcing in mid-Holocene Northern Hemisphere climatic change. Paleoceanography 14, 200-210.

Kienast, S.S., McKay, J.L., 2001. Sea surface temperatures in the subarctic Northeast Pacific reflect millennial-scale climate oscillations during the last 16 kyrs. Geophys Res Lett 28, 1563-1566.

Kienast, M., Kienast, S.S., Calvert, S.E., Eglinton, T.I., Mollenhauer, G., Francois, R., Mix, A.C., 2006. Eastern Pacific cooling and Atlantic overturning circulation during the last deglaciation. Nature 443, 846-849.

Kienast, M., Steinke, S., Stattegger, K., Calvert, S.E., 2001. Synchronous tropical South China Sea SST change and Greenland warming during deglaciation. Science 291, 2132-2134.

Kim, J.H., 2004. GHOST global database for alkenone-derived Holocene sea-surface temperature records. http://www.pangaea.de/Projects/GHOST 
Kim, J.H., Meggers, H., Rimbu, N., Lohmann, G., Freudenthal, T., Muller, P.J., Schneider, R.R., 2007. Impacts of the North Atlantic gyre circulation on Holocene climate off northwest Africa. Geology 35, 387-390.

Kim, J.H., Rimbu, N., Lorenz, S.J., Lohmann, G., Nam, S.I., Schouten, S., Ruhlemann, C., Schneider, R.R., 2004. North Pacific and North Atlantic sea-surface temperature variability during the holocene. Quaternary Science Reviews 23, 2141-2154.

Kim, J.H., Schneider, R.R., Hebbeln, D., Muller, P.J., Wefer, G., 2002a. Last deglacial sea-surface temperature evolution in the Southeast Pacific compared to climate changes on the South American continent. Quaternary Science Reviews 21, 2085-2097.

Kim, J.H., Schneider, R.R., Mulitza, S., Muller, P.J., 2003. Reconstruction of SE trade-wind intensity based on sea-surface temperature gradients in the Southeast Atlantic over the last 25 kyr. Geophys Res Lett 30.

Kim, J.H., Schneider, R.R., Muller, P.J., Wefer, G., 2002b. Interhemispheric comparison of deglacial sea-surface temperature patterns in Atlantic eastern boundary currents. Earth Planet Sc Lett 194, 383-393.

Kirst, G.J., Schneider, R.R., Muller, P.J., von Storch, I., Wefer, G., 1999. Late Quaternary temperature variability in the Benguela Current System derived from alkenones. Quaternary Res 52, 92-103.

Koutavas, A., deMenocal, P.B., Olive, G.C., Lynch-Stieglitz, J., 2006. Mid-Holocene El NinoSouthern Oscillation (ENSO) attenuation revealed by individual foraminifera in eastern tropical Pacific sediments. Geology 34, 993-996.

Koutavas, A., Lynch-Stieglitz, J., Marchitto, T.M., Sachs, J.P., 2002. El Nino-like pattern in ice age tropical Pacific sea surface temperature. Science 297, 226-230.

Koutavas, A., Sachs, J.P., 2008. Northern timing of deglaciation in the eastern equatorial Pacific from alkenone paleothermometry. Paleoceanography 23.

Kubota, Y., Kimoto, K., Tada, R., Oda, H., Yokoyama, Y., Matsuzaki, H., 2010. Variations of East Asian summer monsoon since the last deglaciation based on $\mathrm{Mg} / \mathrm{Ca}$ and oxygen isotope of planktic foraminifera in the northern East China Sea. Paleoceanography 25.

Kucera, M., Rosell-Melé, A., Schneider, R., Waelbroeck, C., Weinelt, M., 2005a. Multiproxy approach for the reconstruction of the glacial ocean surface (MARGO). Quaternary Science Reviews 24, 813-819.

Kucera, M., Weinelt, M., Kiefer, T., Pflaumann, U., Hayes, A., Weinelt, M., Chen, M.-T., Mix, A.C., Barrows, T.T., Cortijo, E., Duprat, J., Juggins, S., Waelbroeck, C., 2005b. Reconstruction of sea-surface temperatures from assemblages of planktonic foraminifera: multi-technique approach based on geographically constrained calibration data sets and its application to glacial Atlantic and Pacific Oceans. Quaternary Science Reviews 24, 951-998. 
Kudrass, H.R., Hofmann, A., Doose, H., Emeis, K., Erlenkeuser, H., 2001. Modulation and amplification of climatic changes in the Northern Hemisphere by the Indian summer monsoon during the past 80 k.y. Geology 29, 63-66.

Labeyrie, L., 1999. Temporal Variability of the surface and deep waters of the North West Atlantic ocean at orbital and millenial scales, In: Clark, P. (Ed.), Mechanisms of Global Climate Change at millenial Time scales. AGU, Washington, pp. 77-98.

Lamy, F., Kaiser, J., Arz, H.W., Hebbeln, D., Ninnemann, U., Timm, O., Timmermann, A., Toggweiler, J.R., 2007. Modulation of the bipolar seesaw in the southeast pacific during Termination 1. Earth Planet Sc Lett 259, 400-413.

Lamy, F., Kaiser, J., Ninnemann, U., Hebbeln, D., Arz, H.W., Stoner, J., 2004. Antarctic timing of surface water changes off Chile and Patagonian ice sheet response. Science 304, 19591962.

Lamy, F., Ruhlemann, C., Hebbeln, D., Wefer, G., 2002. High- and low-latitude climate control on the position of the southern Peru-Chile Current during the Holocene. Paleoceanography 17.

Lea, D.W., Pak, D.K., Belanger, C.L., Spero, H.J., Hall, M.A., Shackleton, N.J., 2006. Paleoclimate history of Galapagos surface waters over the last 135,000 yr. Quaternary Science Reviews 25, 1152-1167.

Lea, D.W., Pak, D.K., Peterson, L.C., Hughen, K.A., 2003. Synchroneity of tropical and high-latitude Atlantic temperatures over the last glacial termination. Science 301, 1361-1364.

Lea, D.W., Pak, D.K., Spero, H.J., 2000. Climate impact of late quaternary equatorial Pacific sea surface temperature variations. Science 289, 1719-1724.

Ledu, D., Rochon, A., de Vernal, A., Barletta, F., St-Onge, G., 2010. Holocene sea ice history and climate variability along the main axis of the Northwest Passage, Canadian Artic. Paleoceanography 25, PA 2213.

Ledu, D., Rochon, A., de Vernal, A., St-Onge, G., 2008. Palynological evidence of Holocene climate change in the eastern Arctic: a possible shift in the Arctic oscillation at the millennial time scale. Canadian Journal of Earth Sciences 45, 1363-1375.

Leduc, G., Schneider, R., Kim, J.H., Lohmann, G., 2010. Holocene and Eemian sea surface temperature trends as revealed by alkenone and $\mathrm{Mg} /$ Ca paleothermometry. Quaternary Science Reviews 29, 989-1004.

Leduc, G., Vidal, L., Tachikawa, K., Rostek, F., Sonzogni, C., Beaufort, L., Bard, E., 2007. Moisture transport across Central America as a positive feedback on abrupt climatic changes. Nature 445, 908-911. 
Lee, K.E., Slowey, N.C., Herbert, T.D., 2001. Glacial sea surface temperatures in the subtropical North Pacific: A comparison of Uk37', delta180, and foraminiferal assemblage temperature estimates. Paleoceanography 16, 268-279.

Levac, E., de Vernal., A., Blake, W. Jr., 2001. Sea-surface conditions in northernmost Baffin Bay during the Holocene: palynological evidence. Journal of Quaternary Science 16, 353-363.

Levi, C., Labeyrie, L., Bassinot, F., Guichard, F., Cortijo, E., Waelbroeck, C., Caillon, N., Duprat, J., de Garidel-Thoron, T., Elderfield, H., 2007. Low-latitude hydrological cycle and rapid climate changes during the last deglaciation. Geochem Geophy Geosy 8.

Lin, D.-C., Liu, C.-H., Fang, T.-H., Tsai, C.-H., Murayama, M., Chen, M.-T., 2006. Millennial-scale changes in terrestrial sediment input and Holocene surface hydrology in the northern South China Sea (IMAGES MD972146). Palaeogeography, Palaeoclimatology, Palaeoecology 236, 56-73.

Lisiecki, L.E., Raymo, M.E., 2005. A Pliocene-Pleistocene stack of 57 globally distributed benthic $\delta 180$ records. Paleoceanography 20, PA1003.

Locarnini, R.A., Mishonov, A.V., Antonov, J.I., Boyer, T.P., Garcia, H.E., Baranova, O.K., Zweng, M.M., Johnson, D.R., 2010. World Ocean Atlas 2009, Volume 1: Temperature., In:

Levitus, S. (Ed.), NOAA Atlas NESDIS 68. U.S. Government Printing Office, Washington, D.C., p. 184.

Lohmann, G., Pfeiffer, M., Laepple, T., Leduc, G., Kim, J.H., 2013. A model-data comparison of the Holocene global sea surface temperature evolution. Clim Past 9, 1807-1839.

Lombard, F., Labeyrie, L., Michel, E., Bopp, L., Cortijo, E., Retailleau, S., Howa, H., Jorissen, F., 2011. Modelling planktic foraminifer growth and distribution using an ecophysiological multi-species approach. Biogeoscience 8, 853-873.

Londeix, L., Herreyre, Y., Turon, J.L., Fletcher, W., 2009. Last Glacial to Holocene hydrology of the Marmara Sea inferred from a dinoflagellate cyst record. Rev Palaeobot Palyno 158, 52 71.

Lückge, A., Mohtadi, M., Ruhlemann, C., Scheeder, G., Vink, A., Reinhardt, L., Wiedicke, M., 2009. Monsoon versus ocean circulation controls on paleoenvironmental conditions off southern Sumatra during the past 300,000 years. Paleoceanography 24 .

Mairesse, A., Goosse, H., Mathiot, P., Wanner, H., Dubinkina, S., 2013. Investigating the consistency between Geoscientific Instrumentation between proxies and models using data assimilation: a mid-Holocene case study. Climate of the Past Discussion 9, 39533991.

Marchal, O., Cacho, I., Stocker, T.F., Grimalt, J.O., Calvo, E., Martrat, B., Shackleton, N., Vautravers, M., Cortijo, E., van Kreveld, S., Andersson, C., Koc, N., Chapman, M., Sbaffi, L., Duplessy, J.C., Sarnthein, M., Turon, J.L., Duprat, J., Jansen, E., 2002. Apparent long-term cooling of 
the sea surface in the northeast Atlantic and Mediterranean during the Holocene. Quaternary Science Reviews 21, 455-483.

Marcott, S.A., Shakun, J.D., Clark, P.U., Mix, A.C., 2013. A Reconstruction of Regional and Global Temperature for the Past 11,300 Years. Science 339, 1198-1201.

MARGO: Waelbroeck, C., Paul, A., Kucera, M., Rosell-Melé, A., Weinelt, M., Schneider, R., Mix, A.C., Abelmann, A., Armand, L., Bard, E., Barker, S., Barrows, T.T., Benway, H., Cacho, I., Chen, M.T., Cortijo, E., Crosta, X., de Vernal, A., Dokken, T., Duprat, J., Elderfield, H., Eynaud, F., Gersonde, R., Hayes, A., Henry, M., Hillaire-Marcel, C., Huang, C.C., Jansen, E., Juggins, S., Kallel, N., Kiefer, T., Kienast, M., Labeyrie, L., Leclaire, H., Londeix, L., Mangin, S., Matthiessen, J., Marret, F., Meland, M., Morey, A.E., Mulitza, S., Pflaumann, U., Pisias, N.G., Radi, T., Rochon, A., Rohling, E.J., Sbaffi, L., Schafer-Neth, C., Solignac, S., Spero, H., Tachikawa, K., Turon, J.L., Members, M.P., 2009. Constraints on the magnitude and patterns of ocean cooling at the Last Glacial Maximum. Nature Geoscience 2, 127-132.

Margreth, S., Gennari, G., Rüggeberg, A., Comas, M.C., Pinheiro, L.M., Spezzaferri, S., 2011. Growth and demise of cold-water coral ecosystems on mud volcanoes in the West Alboran Sea: The messages from the planktonic and benthic foraminifera. Marine Geology 282, 26-39. Martin, P.A., Lea, D.W., 2002. A simple evaluation of cleaning procedures on fossil benthic foraminiferal Mg/Ca. Geochem Geophy Geosy 3. 8401.

Martinson, D.G., Pisias, N.G., Hays, J.D., Imbrie, J., Moore, T.C., Shackleton, N.J., 1987. Age dating and the orbital theory of the ice ages: Development of a high-resolution 0 to 300,000 year chronostratigraphy. Quaternary Research 27. 1-29.

Martrat, B., Grimalt, J.O., Lopez-Martinez, C., Cacho, I., Sierro, F.J., Flores, J.A., Zahn, R., Canals, M., Curtis, J.H., Hodell, D.A., 2004. Abrupt temperature changes in the Western Mediterranean over the past 250,000 years. Science 306, 1762-1765.

Martrat, B., Grimalt, J.O., Shackleton, N.J., de Abreu, L., Hutterli, M.A., Stocker, T.F., 2007. Four climate cycles of recurring deep and surface water destabilizations on the Iberian margin. Science 317, 502-507.

Mashiotta, T.A., Lea, D.W., Spero, H.J., 1999. Glacial-interglacial changes in Subantarctic sea surface temperature and $\delta^{180}$-water using foraminiferal Mg. Earth Planet Sc Lett 170, 417-432.

McKay, J.L., de Vernal, A., Hillaire-Marcel, C., Not, C., Polyak, L., Darby, D., 2008. Holocene fluctuations in Arctic sea ice cover: dinocyst-based reconstructions for the eastern Chukchi Sea. Canadian Journal of Earth Sciences 45, 1377-1397.

Melki, T., Kallel, N., Fontugne, M., 2010. The nature of transitions from dry to wet condition during sapropel events in the Eastern Mediterranean Sea. Palaeogeography, Palaeoclimatology, Palaeoecology 291, 267-285. 
Melki, T., Kallel, N., Jorissen, F.J., Guichard, F., Dennielou, B., Berné, S., Labeyrie, L., Fontugne, M., 2009. Abrupt climate change, sea surface salinity and paleoproductivity in the western Mediterranean Sea (Gulf of Lion) during the last 28 kyr. Palaeogeography, Palaeoclimatology, Palaeoecology 279, 96-113.

Mix, A.C., Ruddiman, W.F., McIntyre, A., 1986. Late Quaternary Paleoceanography of the Tropical Atlantic, 1: Spatial Variability of Annual Mean Sea-Surface Temperatures, 0-20,000 Years Bp. Paleoceanography 1, 43-66.

Mohtadi, M., Hebbeln, D., 2004. Mechanisms and variations of the paleoproductivity off northern Chile $\left(24^{\circ} \mathrm{S}-33^{\circ} \mathrm{S}\right)$ during the last 40,000 years. Paleoceanography 19.

Mohtadi, M., Romero, O.E., Hebbeln, D., 2004. Changing marine productivity off northern Chile during the past 19000 years: a multivariable approach. J Quaternary Sci 19, 347-360.

Mohtadi, M., Steinke, S., Groeneveld, J., Fink, H.G., Rixen, T., Hebbeln, D., Donner, B., Herunadi, B., 2009. Low-latitude control on seasonal and interannual changes in planktonic foraminiferal flux and shell geochemistry off south Java: A sediment trap study. Paleoceanography 24.

Mohtadi, M., Steinke, S., Luckge, A., Groeneveld, J., Hathorne, E.C., 2010. Glacial to Holocene surface hydrography of the tropical eastern Indian Ocean. Earth Planet Sc Lett 292, 8997.

Müller, P.J., Kirst, G., Ruhland, G., von Storch, I., Rosell-Melé, A., 1998. Calibration of the alkenone paleotemperature index U37K' based on core-tops from the eastern South Atlantic and the global ocean $\left(60^{\circ} \mathrm{N}-60^{\circ} \mathrm{S}\right)$. Geochim Cosmochim Ac 62, 1757-1772.

Nürnberg, D., Bijma, J., Hemleben, C., 1996. Assessing the reliability of magnesium in foraminiferal calcite as a proxy for water mass temperatures. Geochim Cosmochim Ac 60, 803-814.

Nürnberg, D., Ziegler, M., Karas, C., Tiedemann, R., Schmidt, M.W., 2008. Interacting Loop Current variability and Mississippi River discharge over the past 400 kyr. Earth and Planetary Science Letters 272, 278-289.

Oppo, D.W., Sun, Y.B., 2005. Amplitude and timing of sea-surface temperature change in the northern South China Sea: Dynamic link to the East Asian monsoon. Geology 33, 785788.

Ostertag-Henning, C., Stax, R., 2000. 26. Data Report: Carbonate records from sites 1012, 1013, 1017, and 1019 and alkenone-based sea-surface temperatures from site 1017. Proceedings of the Ocean Drilling Program, Scientific Results 167.

Pahnke, K., Sachs, J.P., 2006. Sea surface temperatures of southern midlatitudes 0-160 kyr BP. Paleoceanography 21. 
Pahnke, K., Sachs, J.P., Keigwin, L., Timmermann, A., Xie, S.P., 2007. Eastern tropical Pacific hydrologic changes during the past 27,000 years from $\mathrm{D} / \mathrm{H}$ ratios in alkenones. Paleoceanography 22.

Pahnke, K., Zahn, R., Elderfield, H., Schulz, M., 2003. 340,000-year centennial-scale marine record of Southern Hemisphere climatic oscillation. Science 301, 948-952.

Pailler, D., Bard, E., 2002. High frequency palaeoceanographic changes during the past 140000 yr recorded by the organic matter in sediments of the Iberian Margin. Palaeogeography, Palaeoclimatology, Palaeoecology 181, 431-452.

Pelejero, C., Grimalt, J.O., Heilig, S., Kienast, M., Wang, L.J., 1999a. High-resolution UK'37 temperature reconstructions in the South China Sea over the past 220 kyr. Paleoceanography 14, 224-231.

Pelejero, C., Kienast, M., Wang, L., Grimalt, J.O., 1999b. The flooding of Sundaland during the last deglaciation: Imprints in hemipelagic sediments from the southern South China Sea. Earth and Planetary Science Letters, 661-671

Pena, L.D., Cacho, I., Ferretti, P., Hall, M.A., 2008. El Nino-Southern Oscillation-like variability during glacial terminations and interlatitudinal teleconnections. Paleoceanography 23.

Pérez-Folgado, M., Sierro, F.J., Flores, J.A., Cacho, I., Grimalt, J.O., Zahn, R., Shackleton, N., 2003. Western Mediterranean planktonic foraminifera events and millennial climatic variability during the last 70 kyr. Marine Micropaleontology 48, 49-70.

Pérez-Folgado, M., Sierro, F.J., Flores, J.A., Grimalt, J.O., Zahn, R., 2004. Paleoclimatic variations in foraminifer assemblages from the Alboran Sea (Western Mediterranean) during the last 150 ka in ODP Site 977. Marine Geology 212, 113-131.

Peterson, L.C., Haug, G.H., Hughen, K.A., Röhl, U., 2000. Rapid Changes in the Hydrologic Cycle of the Tropical Atlantic During the Last Glacial. Science 290, 1947-1951.

Prahl, F., Muehlhausen, L.A., Zahnle, D.L., 1988. Further evaluation of long-chain alkenones as indicator of paleoceanographic conditions. Geochim Cosmochim Ac 52, 2303-2310.

Prahl, F.G., Mix, A.C., Sparrow, M.A., 2006. Alkenone paleothermometry: Biological lessons from marine sediment records off western South America. Geochim Cosmochim Ac 70, 101117.

Prahl, F.G., Pisias, N., Sparrow, M.A., Sabin, A., 1995. Assessment of Sea-Surface Temperature at 42-Degrees- $\mathrm{N}$ in the California Current over the Last 30,000 Years. Paleoceanography 10, 763-773.

Prahl, F.G., Wakeham, S.G., 1987. Calibration of Unsaturation Patterns in Long-Chain Ketone Compositions for Paleotemperature Assessment. Nature 330, 367-369.

Rasmussen, T.L., Thomsen, E., 2010. Holocene temperature and salinity variability of the Atlantic Water inflow to the Nordic seas. The Holocene 20,1223-1234. 
Rasmussen, T.L., Thomsen, E., 2012. Changes in planktic foraminiferal faunas, temperature and salinity in the Gulf Stream during the last 30,000 years: influence of meltwater via the Mississippi River. Quaternary Science Reviews 33, 42-54.

Rayner, N.A., Parker, D.E., Horton, E.B., Folland, C.K., Alexander, L.V., Rowell, D.P., Kent, E.C., Kaplan, A., 2003. Global analyses of sea surface temperature, sea ice, and night marine air temperature since the late nineteenth century Journal of Geophysical Research 108, D14, 4407.

Reimer, P.J., Baillie, M.G.L., Bard, E., Bayliss, A., Beck, J.W., Blackwell, P.G., Bronk Ramsey, C., Buck, C.E., Burr, G.S., Edwards, R.L., Friedrich, M., Grootes, P.M., Guilderson, T.P., Hajdas, I., Heaton, T.J., Hogg, A.G., Hughen, K.A., Kaiser, K.F., Kromer, B., McCormac, F.G., Manning, S.W., Reimer, R.W., Richards, D.A., Southon, J.R., Talamo, S., Turney, C.S.M., van der Pflicht, J., Weyhenmeyer, C.E., 2009. IntCal09 and Marine09 Radiocarbon Age Calibration Curves, 0-50,000 years cal BP. Radiocarbon 51, 1111-1150.

Regenberg, M., Nürnberg, D., Steph, S., Groeneveld, J., Garbe-Schönberg, D., Tiedemann, R., Dullo, W.-C., 2006. Assessing the effect of dissolution on planktonic foraminiferal Mg/Ca ratios: Evidence from Caribbean core tops. Geochem. Geophys. Geosyst. 7, Q07P15.

Risebrobakken, B., Dokken, T., Smedsrud, L.H., Andersson, C., Jansen, E., Moros, M., Ivanova, E.V., 2011. Early Holocene temperature variability in the Nordic Seas: The role of oceanic heat advection versus changes in orbital forcing. Paleoceanography 26, PA4206.

Rodrigues, T., Grimalt, J.O., Abrantes, F.G., Flores, J.A., Lebreiro, S.M., 2009. Holocene interdependences of changes in sea surface temperature, productivity, and fluvial inputs in the Iberian continental shelf (Tagus mud patch). Geochem Geophy Geosy 10.

Rohling, E.J., Den Dulk, M., Pujol, C., Vergnaud-Grazzini, C., 1995. Abrupt hydrographic change in the Alboran Sea (western Mediterranean) around 8000 yrs BP. Deep Sea Research Part I: Oceanographic Research Papers 42, 1609-1619.

Romero, O.E., Kim, J.H., Donner, B., 2008. Submillennial-to-millennial variability of diatom production off Mauritania, NW Africa, during the last glacial cycle. Paleoceanography 23.

Rosell-Melé, A., Bard, E., Emeis, K.C., Grimalt, J.O., Muller, P., Schneider, R., Bouloubassi, I., Epstein, B., Fahl, K., Fluegge, A., Freeman, K., Goni, M., Guntner, U., Hartz, D., Hellebust, S., Herbert, T., Ikehara, M., Ishiwatari, R., Kawamura, K., Kenig, F., de Leeuw, J., Lehman, S., Mejanelle, L., Ohkouchi, N., Pancost, R.D., Pelejero, C., Prahl, F., Quinn, J., Rontani, J.F., Rostek, F., Rullkotter, J., Sachs, J., Blanz, T., Sawada, K., Schutz-Bull, D., Sikes, E., Sonzogni, C., Ternois, Y., Versteegh, G., Volkman, J.K., Wakeham, S., 2001. Precision of the current methods to measure the alkenone proxy Uk'37and absolute alkenone abundance in sediments: Results of an interlaboratory comparison study. Geochem Geophy Geosy 2. 
Rosell-Melé, A., Eglinton, G., Pflaumann, U., Sarnthein, M., 1995. Atlantic Core-Top Calibration of the U-37(K) Index as a Sea-Surface Paleotemperature Indicator. Geochim Cosmochim Ac 59, 3099-3107.

Rosell-Melé, A., Prahl, F.G., 2013. Seasonality of UK'37 temperature estimates as inferred from sediment trap data. Quaternary Science Reviews 72, 128-136.

Rosenthal, Y., Field, M.P., Sherrell, R.M., 1999. Precise determination of element/calcium ratios in calcareous samples using sector field inductively coupled plasma mass spectrometry. Anal Chem 71, 3248-3253.

Rosenthal, Y., Lohmann, G.P., 2002. Accurate estimations of sea surface temperatures using dissolution-corrected calibrations for $\mathrm{Mg} / \mathrm{Ca}$ paleothermometry. Palaeoceanography 17.

Rosenthal, Y., Lohmann, G.P., Lohmann, K.C., Sherrell, R.M., 2000. Incorporation and Preservation of $\mathrm{Mg}$ in Globigerinoides sacculifer: Implications for Reconstructing the Temperature and ${ }^{18} 0 / 160$ of Seawater. Paleoceanography 15, 135-145.

Rosenthal, Y., Oppo, D.W., Linsley, B.K., 2003. The amplitude and phasing of climate change during the last deglaciation in the Sulu Sea, western equatorial Pacific. Geophysical Research Letters 30.

Rosenthal, Y., Perron-Cashman, S., Lear, C.H., Bard, E., Barker, S., Billups, K., Bryan, M., Delaney, M.L., deMenocal, P.B., Dwyer, G.S., Elderfield, H., German, C.R., Greaves, M., Lea, D.W., Marchitto, T.M., Jr., Pak, D.K., Paradis, G.L., Russell, A.D., Schneider, R.R., Scheiderich, K., Stott, L., Tachikawa, K., Tappa, E., Thunell, R., Wara, M., Weldeab, S., Wilson, P.A., 2004. Interlaboratory comparison study of $\mathrm{Mg} / \mathrm{Ca}$ and $\mathrm{Sr} / \mathrm{Ca}$ measurements in planktonic foraminifera for paleoceanographic research. Geochem. Geophys. Geosyst. 5.

Rouis-Zargouni, I., 2010. Evolution Paleoclimatique et Paleohydrologique de la Mediterranee occidentale au cours des derniers 30000 ans: Contribution des Dinokystes et des Foraminiferes planctoniques. PhD thesis. Université Bordeaux 1, France.

Rouis-Zargouni, I., Turon, J.-L., Londeix, L., Essallami, L., Kallel, N., Sicre, M.-A., 2010. Environmental and climatic changes in the central Mediterranean Sea (Siculo-Tunisian Strait) during the last $30 \mathrm{ka}$ based on dinoflagellate cyst and planktonic foraminifera assemblages. Palaeogeography, Palaeoclimatology, Palaeoecology 285, 17-29.

Rouis-Zargouni, I., Turon, J.L., Londeix, L., Kallel, N., Essallami, L., 2012. The last glacialinterglacial transition and dinoflagellate cysts in the western Mediterranean Sea. Cr Geosci 344, 99-109.

Ruddiman, W.F., Mix, A.C., 1993. The North and Equatorial Atlantic at 9000 and 6000 yr B.P., In: Jr., H.E.W., Kutzbach, J.E., III, T.W., Ruddiman, W.F., Street-Perrott, F.A., Bartlein, P.J. (Eds.), Global Climates since the Last Glacial Maximum. University of Minnesota Press, Minneapolis, pp. 94-124. 
Rühlemann, C., Mulitza, S., Muller, P.J., Wefer, G., Zahn, R., 1999. Warming of the tropical Atlantic Ocean and slowdown of thermohaline circulation during the last deglaciation. Nature $402,511-514$.

Sachs, J.P., 2007. Cooling of Northwest Atlantic slope waters during the Holocene. Geophys Res Lett 34.

Sarnthein, M., Gebhardt, H., Kiefer, T., Kucera, M., Cook, M., Erlenkeuser, H., 2004. Mid Holocene origin of the sea-surface salinity low in the subarctic North Pacific. Quaternary Science Reviews 23, 2089-2099.

Sarnthein, M., Van Kreveld, S., Erlenkeuser, H., Grootes, P.M., Kucera, M., Pflaumann, U., Schulz, M., 2003. Centennial-to-millennial-scale periodicities of Holocene climate and sediment injections off the western Barents shelf, 75 degrees N. Boreas 32, 447-461.

Sawada, K., Handa, N., 1998. Variability of the path of the Kuroshio ocean current over the past 25,000 years. Nature 392, 592-595.

Schefuss, E., Schouten, S., Schneider, R.R., 2005. Climatic controls on central African hydrology during the past 20,000 years. Nature 437, 1003-1006.

Schmidt, M.W., Spero, H.J., Lea, D.W., 2004. Links between salinity variation in the Caribbean and North Atlantic thermohaline circulation. Nature 428, 160-163.

Schneider, B., Leduc, G., Park, W., 2010. Disentangling seasonal signals in Holocene climate trends by satellite-model-proxy integration. Paleoceanography 25.

Schulz, H., Emeis, K.C., Erlenkeuser, H., von Rad, U., Rolf, C., 2002. The Toba volcanic event and interstadial/stadial climates at the marine isotopic stage 5 to 4 transition in the northern Indian Ocean. Quaternary Res 57, 22-31.

Seki, O., Ikehara, M., Kawamura, K., Nakatsuka, T., Ohnishi, K., Wakatsuchi, M., Narita, H., Sakamoto, T., 2004. Reconstruction of paleoproductivity in the Sea of Okhotsk over the last 30 kyr. Paleoceanography 19.

Shackleton, N.J., 2000. The 100,000-Year Ice-Age Cycle identified and found to lag temperature, carbon diaoxid, and orbital eccentricity. Science 289, 1897-1902.

Shakun, J.D., Carlson, A.E., 2010. A global perspective on Last Glacial Maximum to Holocene climate change. Quaternary Science Reviews 29, 1801-1816.

Shiau, L.J., Chen, M.T., Clemens, S.C., Huh, C.A., Yamamoto, M., Yokoyama, Y., 2011. Warm pool hydrological and terrestrial variability near southern Papua New Guinea over the past 50k. Geophys Res Lett 38.

Sicre, M.A., Labeyrie, L., Ezat, U., Duprat, J., Turon, J.L., Schmidt, S., Michel, E., Mazaud, A., 2005. Mid-latitude Southern Indian Ocean response to Northern Hemisphere Heinrich events. Earth Planet Sc Lett 240, 724-731. 
de Simard, A., de Vernal., A., 1998. Distribution des kystes de Alexandrium excavatum dans les sediments récents et postglaciaires des marges est-canadiennes. Géography physique et Quaternaire 52, 361-371.

Skinner, L.C., Elderfield, H., 2005. Constraining ecological and biological bias in planktonic foraminiferal $\mathrm{Mg} / \mathrm{Ca}$ and delta ${ }^{18} \mathrm{O}$ : A multispecies approach to proxy calibration testing. Paleoceanography 20.

Smart, C.W., Maslin, M.A., Dixon, K.E., 2009. NE Atlantic surface water mass changes over the last 15 kyr. Palaeogeogr Palaeocl 282, 58-66.

Solignac, S., de Vernal, A., Hillaire-Marcel, C., 2004. Holocene sea-surface conditions in the North Atlantic-contrasted trends and regimes between the eastern and western sectors (Labrador Sea vs. Iceland Basin). Quaternary Science Reviews 23, 319-334.

Solignac, S., Giraudeau, J., de Vernal, A., 2006. Holocene sea surface conditions in the western North Atlantic: Spatial, and temporal heterogeneities. Paleoceanography 21, PA2004.

Solignac, S., Grelaud, M., de Vernal, A., Giraudeau, J., Moros, M., McCave, I.N., Hoogakker, B., 2008. Reorganization of the upper ocean circulation in the mid-Holocene in the northeastern Atlantic. Canadian Journal of Earth Sciences 45, 1417-1433.

Sonzogni, C., Bard, E., Rostek, F., 1998. Tropical sea-surface temperatures during the last glacial period: A view based on alkenones in Indian Ocean sediments. Quaternary Science Reviews 17, 1185-1201.

Sperling, M., Schmiedl, G., Hemleben, C., Emeis, K.C., Erlenkeuser, H., Grootes, P.M., 2003. Black Sea impact on the formation of eastern Mediterranean sapropel S1? Evidence from the Marmara Sea. Palaeogeogr Palaeocl 190, 9-21.

Steinke, S., Glatz, C., Mohtadi, M., Groeneveld, J., Li, Q.Y., Jian, Z.M., 2011. Past dynamics of the East Asian monsoon: No inverse behaviour between the summer and winter monsoon during the Holocene. Global and Planetary Change 78, 170-177.

Steinke, S., Kienast, M., Groeneveld, J., Lin, L.C., Chen, M.T., Rendle-Buhring, R., 2008. Proxy dependence of the temporal pattern of deglacial warming in the tropical South China Sea: toward resolving seasonality. Quaternary Science Reviews 27, 688-700.

Steinke, S., Kienast, M., Pflaumann, U., Weinelt, M., Stattegger, K., 2001. A high-resolution seasurface temperature record from the tropical South China Sea (16,500-3000 yr BP). Quaternary Res 55, 352-362.

Stott, L., Cannariato, K., Thunell, R., Haug, G.H., Koutavas, A., Lund, S., 2004. Decline of surface temperature and salinity in the western tropical Pacific Ocean in the Holocene epoch. Nature 431, 56-59. 
Sun, Y.B., Oppo, D.W., Xiang, R., Liu, W.G., Gao, S., 2005. Last deglaciation in the Okinawa Trough: Subtropical northwest Pacific link to Northern Hemisphere and tropical climate. Paleoceanography 20.

Telford, R.J., Li, C., Kucera, M., 2013. Mismatch between the depth habitat of planktonic foraminifera and the calibration depth of SST transfer functions may bias reconstructions. Climate of the Past 9, 859-870.

Ternois, Y., Kawamura, K., Keigwin, L., Ohkouchi, N., Nakatsuka, T., 2001. A biomarker approach for assessing marine and terrigenous inputs to the sediments of Sea of Okhotsk for the last 27,000 years. Geochim Cosmochim Ac 65, 791-802.

Ternois, Y., Kawamura, K., Ohkouchi, N., Keigwin, L., 2000. Alkenone sea surface temperature in the Okhotsk Sea for the last 15 kyr. Geochem J 34, 283-293.

Thornalley, D.J.R., Elderfield, H., McCave, I.N., 2009. Holocene oscillations in temperature and salinity of the surface subpolar North Atlantic. Nature 457, 711-714.

Ujiie, Y., Ujiie, H., Taira, A., Nakamura, T., Oguri, K., 2003. Spatial and temporal variability of surface water in the Kuroshio source region, Pacific Ocean, over the past 21,000 years: evidence from planktonic foraminifera. Marine Micropaleontology 49, 335-364.

Visser, K., Thunell, R., Stott, L., 2003. Magnitude and timing of temperature change in the IndoPacific warm pool during deglaciation. Nature 421, 152-155.

von Langen, P.J., Pak, D.K., Spero, H.J., Lea, D.W., 2005. Effects of temperature on $\mathrm{Mg} / \mathrm{Ca}$ in neogloboquadrinid shells determined by live culturing. Geochem Geophy Geosy 6 .

Voronina, E., Polyak, L., de Vernal, A., Peyron, O., 2001. Holocene variations of sea-surface conditions in the southeastern Barents Sea reconstructed from dinoflagellate cyst assemblages. Journal of Quaternary Science 16, 717-726.

Wang, L., Sarnthein, M., Erlenkeuser, H., Grimalt, J.O., Grootes, P.M., Heilig, S., Ivanova, E., Kienast, M., Pelejero, C., Pflaumann, U., 1999. East Asian monsoon climate during the late Quaternary: High-resolution sediment records from the South China Sea. Marine Geology, 245-284.

Weldeab, S., Lea, D.W., Schneider, R.R., Andersen, N., 2007a. 155,000 years of West African monsoon and ocean thermal evolution. Science 316, 1303-1307.

Weldeab, S., Schneider, R.R., Kolling, M., 2006. Deglacial sea surface temperature and salinity increase in the western tropical Atlantic in synchrony with high latitude climate instabilities. Earth and Planetary Science Letters 241, 699-706.

Weldeab, S., Schneider, R.R., Muller, P., 2007b. Comparison of Mg/Ca- and alkenone-based sea surface temperature estimates in the fresh water-influenced Gulf of Guinea, eastern equatorial Atlantic. Geochem Geophy Geosy 8. 
Xie, H., Jia, G., Peng, P. Shao, L., 2007. Sea surface temperature variations in the southwestern South China Sea over the past 160 ka. Acta Oceanologica Sinica, 26 (2), 49-55.

Xu, J., Holbourn, A., Kuhnt, W.G., Jian, Z.M., Kawamura, H., 2008. Changes in the thermocline structure of the Indonesian outflow during Terminations I and II. Earth and Planetary Science Letters 273, 152-162.

Yu, P.-S., Huang, C.-C., Chin, Y., Mii, H.-S., Chen, M.-T., 2006. Late Quaternary East Asian Monsoon variability in the South China Sea: Evidence from planktonic faunal and hydrographic gradient records. Palaeogeography, Palaeoclimatology, Palaeoecology 236, 74-90.

Zhao, M., Beveridge, N.A.S., Shackleton, N.J., Sarnthein, M., Eglinton, G., 1995. Molecular Stratigraphy of Cores Off Northwest Africa - Sea-Surface Temperature History over the Last 80 Ka. Paleoceanography 10, 661-675.

Zhao, M.X., Huang, C.Y., Wang, C.C., Wei, G.J., 2006. A millennial-scale U37K sea-surface temperature record from the South China Sea $\left(8^{\circ} \mathrm{N}\right)$ over the last $150 \mathrm{kyr}$ : Monsoon and sea-level influence. Palaeogeography, Palaeoclimatology, Palaeoecology 236, 39-55. 